\title{
Rigid Cohomology and de Rham-Witt Complexes
}

\author{
Pierre Berthelot
}

Dedicated to Francesco Baldassarri, on his sixtieth birthday

ABSTRACT - Let $k$ be a perfect field of characteristic $p>0, W_{n}=W_{n}(k)$. For separated $k$-schemes of finite type, we explain how rigid cohomology with compact supports can be computed as the cohomology of certain de Rham-Witt complexes with coefficients. This result generalizes the classical comparison theorem of Bloch-Illusie for proper and smooth schemes. In the proof, the key step is an extension of the Bloch-Illusie theorem to the case of cohomologies relative to $W_{n}$ with coefficients in a crystal that is only assumed to be flat over $W_{n}$.

\section{Introduction}

Let $k$ be a perfect field of characteristic $p>0, W_{n}=W_{n}(k)$ (for all $n \geq 1), W=W(k), K=\operatorname{Frac}(W)$. If $X$ is a proper and smooth scheme over $k$, and if $W \Omega_{X}^{\bullet}=\lim _{\overleftarrow{n}} W_{n} \Omega_{X}^{\bullet}$ denotes the de Rham-Witt complex of $X$, the classical comparison theorem between crystalline and de Rham-Witt cohomologies ([8, III, Th. 2.1], [14, II, Th. 1.4]) provides canonical isomorphisms

$$
\begin{aligned}
H_{\mathrm{crys}}^{*}\left(X / W_{n}\right) & \stackrel{\sim}{\longrightarrow} H^{*}\left(X, W_{n} \Omega_{X}^{\bullet},\right. \\
H_{\mathrm{crys}}^{*}(X / W) & \stackrel{\sim}{\longrightarrow} H^{*}\left(X, W \Omega_{X}^{\bullet}\right), \\
H_{\mathrm{crys}}^{*}(X / W)_{K} & \stackrel{\sim}{\longrightarrow} H^{*}\left(X, W \Omega_{X, K}^{\bullet}\right),
\end{aligned}
$$

where the subscript ${ }_{K}$ denotes tensorization with $K$. In particular, the

(*) Indirizzo dell'A.: IRMAR, Université de Rennes 1, Campus de Beaulieu, 35042 Rennes cedex, France.

E-mail: pierre.berthelot@univ-rennes1.fr 
latter allows to study the slope decomposition of crystalline cohomology under the Frobenius action, thanks to the degeneracy of the spectral sequence defined by the filtration of $W \Omega_{X}^{\bullet}$ by the subcomplexes $W \Omega_{\bar{X}}^{\geq i}$ [14, II, Cor. 3.5].

In this article, our main goal is to generalize the isomorphism (1.1.3) to the case of a separated $k$-scheme of finite type. Then $H_{\text {crys }}^{*}(X / W)_{K}$ is no longer in general a good cohomology theory, but we may use instead rigid cohomology with compact supports, which coincides with $H_{\text {crys }}^{*}(X / W)_{K}$ when $X$ is proper and smooth, while retaining all the standard properties of a topological cohomology theory. The key case is the case of a proper $k$ scheme, as the non proper case can be reduced to the proper one using a mapping cone description. We will therefore assume for the rest of the introduction that $X$ is proper.

When $X$ is singular, the classical theory of the de Rham-Witt complex can no longer be directly applied to $X$. Instead, we will consider a closed immersion of $X$ into a smooth $k$-scheme $Y$ of finite type, and we will use on $Y$ de Rham-Witt complexes with coefficients in crystals, as introduced by Étesse in [11]. More precisely, we will construct on $Y$ a $W \mathcal{O}_{Y, K^{-}}$algebra $\mathcal{A}_{X, Y}^{W}$, supported in $X$ and closely related to the algebra of analytic functions on the tube of $X$ in $Y$ when $Y$ admits a lifting as a smooth $p$-adic formal scheme $Y$ over $W$. This algebra is endowed with a de Rham-Witt connection, which extends so as to define a complex $\mathcal{A}_{X, Y}^{W} \widehat{\otimes}_{W \mathcal{O}_{Y}} W \Omega_{Y}^{\bullet}$ (the tensor product being completed for the canonical topology of the de RhamWitt complex). Our main result, which is part of Theorem 5.2, is then the existence of a functorial isomorphism

$$
H_{\text {rig }}^{*}(X / K) \stackrel{\sim}{\longrightarrow} H^{*}\left(Y, \mathcal{A}_{X, Y}^{W} \widehat{\otimes}_{W \mathcal{O}_{Y}} W \Omega_{Y}^{\bullet}\right)
$$

which coincides with (1.1.3) when $X$ is smooth and $Y=X$.

A key ingredient in the proof of Theorem 5.2 is the use of Theorem 3.6 and of its Corollary 3.8, which provide generalizations of the comparison isomorphisms (1.1.1) and (1.1.2) to the case of cohomologies of a smooth $k$ scheme $Y$ with coefficients in a crystal in $\mathcal{O}_{Y / W_{n}}$-modules (resp. $\mathcal{O}_{Y / W^{-}}$ modules) $\mathcal{E}$. Such results have been previously obtained by Étesse [11, Théorème 2.1] and Langer-Zink [18, Theorem 3.8] when $\mathcal{E}$ is flat over $\mathcal{O}_{Y / W_{n}}$ (resp. $\left.\mathcal{O}_{Y / W}\right)$. However, this assumption is not verified in our situation, since we work on the smooth scheme $Y$ with crystals supported in a closed subscheme $X \subset Y$. We give here a proof of the comparison theorem with coefficients in $\mathcal{E}$ that only requires $\mathcal{E}$ to be flat over $W_{n}$, in the sense of Definition 2.3 (resp. quasi-coherent and flat relative to $W$ ). It 
provides under these assumptions functorial isomorphisms

$$
\begin{aligned}
H_{\text {crys }}^{*}\left(Y / W_{n}, \mathcal{E}\right) & \stackrel{\sim}{\longrightarrow} H^{*}\left(Y, \mathcal{E}_{n}^{W} \otimes_{W_{n} \mathcal{O}_{Y}} W_{n} \Omega_{Y}^{\bullet}\right), \\
H_{\text {crys }}^{*}(Y / W, \mathcal{E}) & \stackrel{\sim}{\longrightarrow} H^{*}\left(Y, \widehat{\mathcal{E}}^{W} \widehat{\otimes}_{W \mathcal{O}_{Y}} W \Omega_{Y}^{\bullet}\right),
\end{aligned}
$$

where $\mathcal{E}_{n}^{W}$ is the evaluation of the crystal $\mathcal{E}$ on the PD-thickening $\left(Y, W_{n} Y\right)$ (resp. $\widehat{\mathcal{E}}^{W}=\lim _{\overleftarrow{n}} \mathcal{E}_{n}^{W}$ ). As a first application, we get that, when the immersion $X \hookrightarrow Y$ is regular (in particular, when $X$ is smooth), there exists functorial isomorphisms

$$
\begin{gathered}
H_{\text {crys }}^{*}\left(X / W_{n}\right) \stackrel{\sim}{\longrightarrow} H^{*}\left(Y, \mathcal{P}_{X, Y, n}^{W} \otimes_{W_{n} \mathcal{O}_{Y}} W_{n} \Omega_{Y}^{\bullet}\right), \\
H_{\text {crys }}^{*}(X / W) \stackrel{\sim}{\longrightarrow} H^{*}\left(Y, \widehat{\mathcal{P}}_{X, Y}^{W} \widehat{\otimes}_{W \mathcal{O}_{Y}} W \Omega_{Y}^{\bullet}\right),
\end{gathered}
$$

where $\mathcal{P}_{X, Y, n}^{W}$ is the divided power envelope of $\operatorname{Ker}\left(W_{n} \mathcal{O}_{Y} \rightarrow W_{n} \mathcal{O}_{X}\right)$ with compatibility with the canonical divided powers of $V W_{n-1} \mathcal{O}_{Y}$, and $\widehat{\mathcal{P}}_{X, Y}^{W}=\lim _{\overleftarrow{n}} \mathcal{P}_{X, Y, n}^{W}$

Note that, even if $X$ is smooth, the possibility of computing the crystalline cohomology of $X$ using the de Rham-Witt complex of a smooth embedding was not previously known. Our results imply in particular that, as objects of the derived category $D^{\mathrm{b}}(X, K)$, the complexes $\mathcal{P}_{X, Y, n}^{W} \otimes_{W_{n} \mathcal{O}_{Y}} W_{n} \Omega_{Y}^{\bullet}, \widehat{\mathcal{P}}_{X, Y}^{W} \widehat{\otimes}_{W \mathcal{O}_{Y}} W \Omega_{Y}^{\bullet}$, and $\mathcal{A}_{X, Y}^{W} \widehat{\otimes}_{W \mathcal{O}_{Y}} W \Omega_{Y}^{\bullet}$ do not depend, up to canonical isomorphism, on the embedding $X \hookrightarrow Y$. It would be interesting to have a direct proof of this fact in the form of appropriate Poincaré Lemmas.

We now briefly describe the content of each section.

In section 2, we prove some Tor-independence properties of the sheaves of Witt differentials $W_{n} \Omega_{Y}^{j}$ on a smooth $k$-scheme $Y$, and, more generally, of the graded modules associated to their canonical filtration or to their $p$ adic filtration. Specifically, we show in Theorems 2.6 and 2.10 the vanishing of the higher Tor's involving such sheaves and the evaluation on the PDthickening $\left(Y, W_{n} Y\right)$ of a crystal on $Y / W_{n}$ that is flat over $W_{n}$ - a result that may seem surprising at first sight, given the intricate structure of the sheaves of Witt differentials. The flatness assumption allows to reduce to similar statements for the reduction modulo $p$ of the crystal. The key point is then the existence of a filtration of the crystal such that the corresponding graded pieces have $p$-curvature 0 . Thanks to Cartier's descent, this allows to write them as Frobenius pullbacks, and it is then possible to conclude using the local freeness results proved in [14] when the graded 
modules associated to $W_{n} \Omega_{Y}^{j}$ are viewed as $\mathcal{O}_{Y}$-modules through an appropriate Frobenius action.

We use these results in section 3 to prove the comparison theorem between crystalline and de Rham-Witt cohomologies with coefficients in a crystal that is flat over $W_{n}$ (Theorem 3.6). As in the constant coefficient case, the proof proceeds by reduction to the associated graded complexes for the $p$-adic and for the canonical filtration. The Tor-independence results of the previous sections allow to reduce to the fact that, in the constant coefficient case, one gets quasi-isomorphisms between complexes that can be viewed as strictly perfect complexes of $\mathcal{O}_{Y}$-modules when $\mathcal{O}_{Y}$ acts through an appropriate power of Frobenius. The section ends with the above mentionned application to crystalline cohomology for regularly embedded subschemes.

We begin section 4 by recalling the definition of rigid cohomology for a proper $k$-scheme $X$. Given a closed immersion of $X$ in a smooth $p$-adic formal scheme $\mathrm{P}$ over $W$, let $\mathcal{A}_{X, \mathrm{P}}$ be the direct image by specialization of the sheaf of analytic functions on the tube of $X$ in $\mathbb{P}$. We explain how $\mathcal{A}_{X, \mathrm{P}}$ can be identified with the inverse limit of the completed divided power envelopes (tensorized with $K$ ) of the ideals of the infinitesimal neighbourhoods of $X$ in $P$. We also give a variant of this result in which these envelopes are replaced by their quotients by the ideal of $p$-torsion sections. If $X$ is proper over $k$ and is embedded as a closed subscheme in a smooth $k$-scheme $Y$, we derive from this construction an isomorphism between the rigid cohomology of $X$ and the derived inverse limit of the crystalline cohomologies (tensorized with $K$ ) of the infinitesimal neighbourhoods of $X$ in $Y$ (Theorem 4.7).

In section 5, we keep these last hypotheses, and we use the crystalline nature of the previous constructions to define two inverse systems of $W \mathcal{O}_{Y, K}$-algebras canonically associated to $X \hookrightarrow Y$, with isomorphic inverse limits. By definition, this common inverse limit is the $W \mathcal{O}_{Y, K}$-algebra $\mathcal{A}_{X, Y}^{W}$ entering in the isomorphism (1.1.4). To define (1.1.4) and to prove Theorem 5.2 , we combine the isomorphism of the previous section, which identifies the rigid cohomology of $X$ with the limit of the crystalline cohomologies of its infinitesimal neighbourhoods in $Y$, with an isomorphism derived from the comparison theorem 3.6, which gives an identification of this limit with the cohomology of the complex $\mathcal{A}_{X, Y}^{W} \widehat{\otimes}_{W \mathcal{O}_{Y}} W \Omega_{Y}^{\bullet}$. The first identification uses the description of $\mathcal{A}_{X, Y}^{W}$ as a limit of PD-envelopes, while, because of the $W_{n}$-flatness assumption in Theorem 3.6, the second one uses the second description given in section 4 , based on $p$-torsion free quotients of PDenvelopes. We end the section by extending (1.1.4) to rigid cohomology with compact supports for open subschemes of $X$. 
To conclude this article, we explain the relation between the isomorphism (1.1.4) and the isomorphism [5, (1.3)], which identifies the slope $<1$ part of rigid cohomology with Witt vector cohomology. As a consequence, we obtain that the part of slope $\geq 1$ of $H_{\text {rig }}^{*}(X / K)$ can be identified with the cohomology of a subcomplex of $\mathcal{A}_{X, Y}^{W} \widehat{\otimes}_{W \mathcal{O}_{Y}} W \Omega_{Y}^{\bullet}$. We hope that Theorem 5.2 can also be used to provide a description of parts of higher slopes in $H_{\text {rig }}^{*}(X / K)$, but this is still an open question at this point.

\section{General conventions}

1) In the whole article, we denote by $k$ a perfect field of characteristic $p$, by $W_{n}=W_{n}(k)$ (for $n \geq 1$ ) and $W=W(k)$ the usual rings of Witt vectors with coefficients in $k$, and by $K$ the fraction field of $W$. Formal schemes over $W$ are always supposed to be $p$-adic formal schemes.

2) If $Y$ is a $k$-scheme, we call crystal on $Y / W_{n}$ (resp. $Y / W$ ) a crystal in $\mathcal{O}_{Y / W_{n}}$-modules (resp. $\mathcal{O}_{Y / W}$-modules) on the usual crystalline site $\operatorname{Crys}\left(Y / W_{n}\right)(\operatorname{resp} . \operatorname{Crys}(Y / W))$ [7, Def. 6.1].

3) From section 3 till the end of the article, we assume for simplicity that all schemes and formal schemes under consideration are quasi-compact and separated.

\section{Tor-independence properties of the de Rham-Witt complex}

Let $Y$ be a smooth $k$-scheme. We prove in this section Tor-independence results between the sheaves of de Rham-Witt differential forms $W_{n} \Omega_{Y}^{j}$ and the evaluation on the Witt thickenings $\left(Y, W_{n} Y\right)$ of a crystal on $Y / W_{n}$ that is flat over $W_{n}$ (see Definition 2.3).

2.1. Let $\mathcal{E}$ be a crystal on $Y / W_{n}$ for some $n \geq 1$ (resp. a crystal on $Y / W)$. Recall that:

a) For each divided powers thickening $U \hookrightarrow T$, where $U \subset Y$ is an open subset and $T$ a $W_{n}$-scheme (resp. a $W_{n}$-scheme for some $n$ ), $\mathcal{E}$ defines an $\mathcal{O}_{T}$-module $\mathcal{E}_{T}$, which we call the evaluation of $\mathcal{E}$ on $T$.

b) For each morphism of thickenings $\left(U^{\prime}, T^{\prime}\right) \rightarrow(U, T)$, defined by a $W_{n}$-PD-morphism $v: T^{\prime} \rightarrow T$, the transition morphism

$$
v^{*} \mathcal{E}_{T} \rightarrow \mathcal{E}_{T^{\prime}},
$$

defined by the structure of $\mathcal{E}$ as a sheaf of $\mathcal{O}_{Y / W_{n}}$-modules (resp. $\mathcal{O}_{Y / W^{-}}$ modules) on the crystalline site, is an isomorphism. 
We will consider more specifically two families of thickenings. The first one is the family of thickenings $\left(U, U_{n}\right)$, where $U \subset Y$ is an open subset and $U_{n}$ is a smooth lifting of $U$ over $W_{n}$ (which always exist when $U$ is affine). When $U=Y$ and a smooth lifting $Y_{n}$ has been given, we will simply denote by $\mathcal{E}_{n}$ the $\mathcal{O}_{Y_{n}}$-module $\mathcal{E}_{Y_{n}}$. From the crystal structure of $\mathcal{E}, \mathcal{E}_{n}$ inherits an integrable connection $\nabla_{n}: \mathcal{E}_{n} \rightarrow \mathcal{E}_{n} \otimes \Omega_{Y_{n} / W_{n}}^{1}$, which is quasinilpotent [7, Def. 4.10]. This construction is functorial in $\mathcal{E}$, and defines an equivalence between the category of crystals on $Y / W_{n}$ and the category of $\mathcal{O}_{Y_{n}}$-modules endowed with an integrable and quasi-nilpotent connection relative to $W_{n}$.

If $\mathcal{E}$ is a crystal on $Y / W$ and $Y$ is lifted as a smooth formal scheme $Y$ over $W$, with reduction $Y_{n}$ over $W_{n}, \mathcal{E}$ can be viewed as a compatible family of crystals on $Y / W_{n}$ for all $n$. Thus, $\mathcal{E}$ defines for all $n$ an $\mathcal{O}_{Y_{n}}$-module $\mathcal{E}_{n}$ endowed with an integrable connection $\nabla_{n}$ such that $\mathcal{E}_{n} \simeq \mathcal{E}_{n+1} / p^{n} \mathcal{E}_{n+1}$ as a module with connection. Then the $\mathcal{O}_{Y}$-module $\widehat{\mathcal{E}}=\lim _{\overleftarrow{n}} \mathcal{E}_{n}$ has a connection $\nabla=\lim _{\overleftarrow{n}} \nabla_{n}$, and one gets in this way an equivalence of categories between the category of crystals on $Y / W$ and the category of $p$-adically separated and complete $\mathcal{O}_{Y}$-modules endowed with an integrable and topologically quasi-nilpotent connection.

The second family of thickenings we are going to use is provided by the immersions $Y \hookrightarrow W_{n} Y:=\left(|Y|, W_{n} \mathcal{O}_{Y}\right)$, which are divided powers thickenings thanks to the canonical divided powers of the ideal $V W_{n-1} \mathcal{O}_{Y}$ (defined by $(V x)^{[i]}=\left(p^{i-1} / i !\right) V\left(x^{i}\right)$ for all $\left.i \geq 1\right)$. Thus, evaluating $\mathcal{E}$ on $\left(Y, W_{n} Y\right)$ defines a $W_{n} \mathcal{O}_{Y}$-module, which will be denoted by $\mathcal{E}_{n}^{W}$. For $n^{\prime} \leq n$, the closed immersion $W_{n^{\prime}} Y \hookrightarrow W_{n} Y$ defines a morphism of PD-thickenings of $Y$, hence the crystal structure of $\mathcal{E}$ provides a homomorphism $\mathcal{E}_{n}^{W} \rightarrow \mathcal{E}_{n^{\prime}}^{W}$, the linear factorization of which is an isomorphism

$$
W_{n^{\prime}} \mathcal{O}_{Y} \otimes_{W_{n} \mathcal{O}_{Y}} \mathcal{E}_{n}^{W} \stackrel{\sim}{\longrightarrow} \mathcal{E}_{n^{\prime}}^{W} .
$$

If $\mathcal{E}$ is a crystal on $Y / W$, these homomorphisms turn the family of $W_{n} \mathcal{O}_{Y^{-}}$ modules $\left(\mathcal{E}_{n}^{W}\right)_{n \geq 1}$ into an inverse system of $W_{n} \mathcal{O}_{Y}$-modules.

2.2. Let $\sigma: W \rightarrow W$ be the Frobenius automorphism of $W$. We now assume that we are given a smooth formal scheme $Y$ lifting $Y$ over $W$, with reduction $Y_{n}$ over $W_{n}$. We assume in addition that $Y$ is endowed with a $\sigma$ semi-linear morphism $F: \mathbb{Y} \rightarrow Y$ lifting the absolute Frobenius endomorphism of $Y$. As $\mathcal{O}_{Y}$ is $p$-torsion free, the homomorphism $F: \mathcal{O}_{Y} \rightarrow \mathcal{O}_{Y}$ defines a section $s_{F}: \mathcal{O}_{Y} \rightarrow W \mathcal{O}_{Y}$ of the reduction homomorphism 
$W \mathcal{O}_{Y} \rightarrow \mathcal{O}_{Y}$, characterized by the fact that $w_{i}\left(s_{F}(x)\right)=F^{i}(x)$ for any $x \in \mathcal{O}_{Y}$ and any ghost component $w_{i}[14,0,1.3]$. Composing $s_{F}$ with the reduction homomorphisms and factorizing, we get for all $n \geq 1$ a homomorphism

$$
t_{F}: \mathcal{O}_{Y_{n}} \rightarrow W_{n} \mathcal{O}_{Y}
$$

These homomorphisms are compatible with the reduction maps when $n$ varies, and functorial in an obvious way with respect to the couple $(\mathbb{Y}, F)$.

The morphism $t_{F}$ is a PD-morphism, because the canonical divided powers of $V W_{n-1} \mathcal{O}_{Y}$ extend the natural divided powers of $(p)$. Therefore, it defines a morphism of thickenings of $Y$, and, for any crystal $\mathcal{E}$ on $Y / W_{n}$, we get a canonical isomorphism

$$
W_{n} \mathcal{O}_{Y} \otimes_{\mathcal{O}_{Y_{n}}} \mathcal{E}_{n} \stackrel{\sim}{\longrightarrow} \mathcal{E}_{n}^{W},
$$

where the scalar extension is taken by means of the homomorphism $t_{F}$.

We will need the following condition on $\mathcal{E}$ :

DeFinition 2.3. Let $Y$ be a smooth $k$-scheme.

(i) If $\mathcal{E}$ is a crystal on $Y / W_{n}$, we say that $\mathcal{E}$ is flat over $W_{n}$ if, for any smooth lifting $U_{n}$ over $W_{n}$ of an open subset $U \subset Y$, the evaluation $\mathcal{E}_{U_{n}}$ of $\mathcal{E}$ on $U_{n}$ is flat over $W_{n}$.

(ii) If $\mathcal{E}$ is a crystal on $Y / W$, we say that $\mathcal{E}$ is flat over $W$ if, for all $n$, the induced crystal on $Y / W_{n}$ is flat over $W_{n}$.

For any open subset $U \subset Y$, two smooth liftings $U_{n}, U_{n}^{\prime}$ of $U$ over $W_{n}$ are locally isomorphic, and such local isomorphisms extend canonically to the evaluations on $U_{n}$ and $U_{n}^{\prime}$ of a crystal. Therefore, $\mathcal{E}$ is flat over $W_{n}$ if and only if there exists an open covering $U_{\alpha}$ of $Y$ and, for all $\alpha$, a smooth lifting $U_{\alpha, n}$ of $U_{\alpha}$ over $W_{n}$ such that the evaluation $\mathcal{E}_{\alpha, n}$ of $\mathcal{E}$ on $U_{\alpha, n}$ is flat over $W_{n}$.

Similarly, when $\mathcal{E}$ is a crystal on $Y / W, \mathcal{E}$ is flat over $W$ if and only if there exists an open covering $U_{\alpha}$ of $Y$ and, for all $\alpha$, a lifting $U_{\alpha}$ of $U_{\alpha}$ as a smooth formal scheme over $W$ such that the corresponding Zariski sheaf $\widehat{\mathcal{E}}_{\alpha}=\lim _{\overleftarrow{n}} \mathcal{E}_{\alpha, n}$ on $\cup_{\alpha}$ is $p$-torsion free.

If $\mathcal{E}$ is flat over $W_{n}$ (resp. $W$ ), then its restriction to $\operatorname{Crys}\left(Y / W_{i}\right)$ is flat over $W_{i}$ for any $i \leq n$ (resp. any $i$ ). If $\mathcal{E}$ is flat as an $\mathcal{O}_{Y / W_{n}}$-module (resp. as an $\mathcal{O}_{Y / W}$-module), then $\mathcal{E}$ is flat over $W_{n}$ (resp. over $W$ ).

We now begin our study of the Tor-independence properties between the sheaves $W_{n} \Omega_{Y}^{j}$ and the evaluation of a crystal that is flat over $W_{n}$. We recall first from [14, I, 3.1] that, for $i \in Z$, the $i$-th step of the canonical 
filtration of $W_{n} \Omega_{Y}^{j}$ (resp. $W \Omega_{Y}^{j}$ ) is the sub- $W_{n} \mathcal{O}_{Y}$-module (resp. sub- $W \mathcal{O}_{Y^{-}}$ module) defined by

$$
\begin{gathered}
\operatorname{Fil}^{i} W_{n} \Omega_{Y}^{j}= \begin{cases}W_{n} \Omega_{Y}^{j} & \text { if } i \leq 0, \\
\operatorname{Ker}\left(W_{n} \Omega_{Y}^{j} \rightarrow W_{i} \Omega_{Y}^{j}\right) & \text { if } 1 \leq i \leq n, \\
0 & \text { if } i>n\end{cases} \\
\left(\text { resp. Fil } W \Omega_{Y}^{j}=\left\{\begin{array}{ll}
W \Omega_{Y}^{j} & \text { if } i \leq 0, \\
\operatorname{Ker}\left(W \Omega_{Y}^{j} \rightarrow W_{i} \Omega_{Y}^{j}\right) & \text { if } i \geq 1
\end{array}\right) .\right.
\end{gathered}
$$

We denote

$$
\begin{aligned}
\operatorname{gr}^{i} W_{n} \Omega_{Y}^{j} & =\mathrm{Fil}^{i} W_{n} \Omega_{Y}^{j} / \mathrm{Fil}^{i+1} W_{n} \Omega_{Y}^{j}, \\
\operatorname{gr}^{i} W \Omega_{Y}^{j} & =\mathrm{Fil}^{i} W \Omega_{Y}^{j} / \mathrm{Fil}^{i+1} W \Omega_{Y}^{j} .
\end{aligned}
$$

By construction, the canonical homomorphism

$$
\operatorname{gr}^{i} W \Omega_{Y}^{j} \longrightarrow \operatorname{gr}^{i} W_{n} \Omega_{Y}^{j}
$$

is an isomorphism for $i<n$. Note that, under the assumptions of 2.2, the homomorphism $t_{F}$ defined in (2.2.1) endows the sheaves $\mathrm{Fil}^{i} W_{r} \Omega_{Y}^{j}$ and $\mathrm{gr}^{i} W_{r} \Omega_{Y}^{j}$ with an $\mathcal{O}_{Y_{n}}$-module structure for $1 \leq r \leq n$ and all $i, j$.

The following lemma allows to relate the reduction modulo $p$ of $t_{F}$ with the usual Frobenius endomorphism.

Lemma 2.4. Let $A$ be a commutative ring without p-torsion, $F: A \rightarrow A$ a ring homomorphism lifting the absolute Frobenius endomrphism of $A / p A$, and $s_{F}: A \rightarrow W(A)$ the ring homomorphism such that $w_{i}\left(s_{F}(x)\right)=F^{i}(x)$ for all $x \in A$ and all $i \geq 0[14,0,1.3]$. Let $n \geq 1$ be an integer, $A_{n}=A / p^{n} A, t_{F}: A_{n} \rightarrow W_{n}\left(A_{1}\right)$ the factorization of $s_{F}$ as in (2.2.1), and $\bar{t}_{F}: A_{1} \rightarrow W_{n}\left(A_{1}\right) / p W_{n}\left(A_{1}\right)$ the reduction modulo $p$ of $t_{F}$. Denote by

$$
\bar{F}: A_{1} \simeq W_{n}\left(A_{1}\right) / V W_{n-1}\left(A_{1}\right) \rightarrow W_{n}\left(A_{1}\right) / p W_{n}\left(A_{1}\right)
$$

the homomorphism induced by the action of $F$ on $W_{n}\left(A_{1}\right) / p W_{n}\left(A_{1}\right)$. Then $\bar{F}$ is equal to the composition

$$
A_{1} \stackrel{F}{\longrightarrow} A_{1} \stackrel{\bar{t}_{F}}{\longrightarrow} W_{n}\left(A_{1}\right) / p W_{n}\left(A_{1}\right),
$$

where the first homomorphism $F$ is the absolute Frobenius endomorphism of $A_{1}$. 
Proof. It suffices to prove the equality of the morphisms obtained by composing $\bar{F}$ and $\bar{t}_{F} \circ F$ with the surjection $A \rightarrow A_{1}$. By construction, there is a commutative diagram

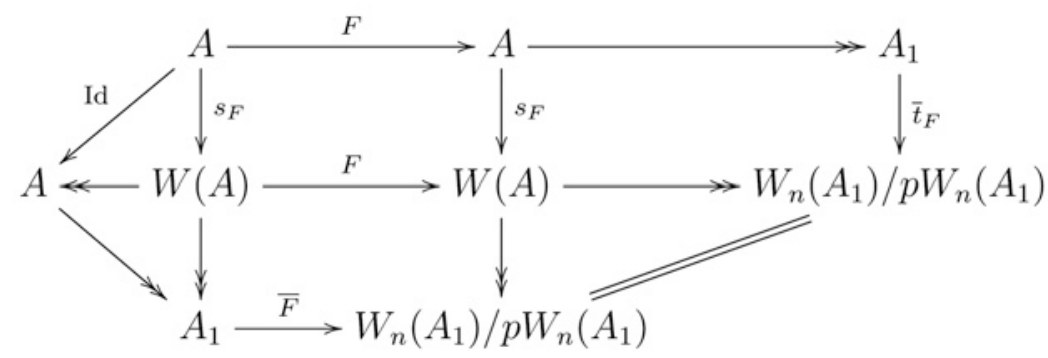

in which the unlabelled arrows are the canonical surjections. Note that the commutativity of the left upper square (in which the upper $F$ is the given homomorphism and the lower one the canonical Frobenius endomorphism of $W(A)$ ) is a consequence of the definition of $s_{F}$ [14, 0 , 1.3]. The lemma follows from the equality of the exterior paths of the diagram.

LEMMA 2.5. Let $Y$ be a smooth $k$-scheme, such that there exists a smooth formal scheme $Y$ over $W$ lifting $Y$ and a semi-linear endomorphism $F$ of $Y$ lifting the absolute Frobenius endomorphism of $Y$. For some $n \geq 1$, let $\mathcal{E}$ be a crystal on $Y / W_{n}$, flat over $W_{n}$. Let $Y_{n}$ be the reduction of $Y$ over $W_{n}$ and $\mathcal{E}_{n}$ the evaluation of $\mathcal{E}$ on $Y_{n}$. Then, for the $\mathcal{O}_{Y_{n}}$-module structure defined on $W_{n} \mathcal{O}_{Y}$-modules by $t_{F}$,

$$
\mathcal{T}_{0} r_{q}^{\mathcal{O}_{Y_{n}}}\left(\mathcal{E}_{n}, \operatorname{Fil}^{i} W_{r} \Omega_{Y}^{j}\right)=\mathcal{T}_{o} r_{q}^{\mathcal{O}_{Y_{n}}}\left(\mathcal{E}_{n}, \operatorname{gr}^{i} W_{r} \Omega_{Y}^{j}\right)=0
$$

for $q \geq 1,1 \leq r \leq n$, and all $i, j$.

In particular, when $W_{n} \mathcal{O}_{Y}$ is viewed as an $\mathcal{O}_{Y_{n}}$-algebra through $t_{F}, \mathcal{E}_{n}$ and $W_{n} \mathcal{O}_{Y}$ are Tor-independent over $\mathcal{O}_{Y_{n}}$.

Proof. The canonical filtration of $W_{r} \Omega_{Y}^{i}$ is discrete and codiscrete, so it suffices to prove the vanishing of $\mathcal{T}_{0} r_{q}^{\mathcal{O}_{Y_{n}}}\left(\mathcal{E}_{n}, \operatorname{gr}^{i} W_{r} \Omega_{Y}^{j}\right)$. Moreover, we may assume that $r=n$, since $\mathrm{gr}^{i} W_{r} \Omega_{Y}^{j}=0$ for $i<0$ or $i \geq r$, and the natural map $\operatorname{gr}^{i} W_{n} \Omega_{Y}^{j} \rightarrow \operatorname{gr}^{i} W_{r} \Omega_{Y}^{j}$ is an isomorphism for $0 \leq i<r \leq n$.

As $\operatorname{gr}^{i} W_{n} \Omega_{Y}^{j}$ is annihilated by $p$, its $\mathcal{O}_{Y_{n}}$-module structure is also given by the composition $\mathcal{O}_{Y_{n}} \rightarrow \mathcal{O}_{Y} \stackrel{\bar{t}_{F}}{\rightarrow} W_{n} \mathcal{O}_{Y} / p W_{n} \mathcal{O}_{Y}$, where $\bar{t}_{F}$ is the reduction 
of $t_{F} \bmod p$. Therefore, we obtain isomorphisms

$$
\mathcal{E}_{n} \underset{\mathcal{O}_{Y_{n}}}{\stackrel{\mathrm{L}}{\otimes}} \operatorname{gr}^{i} W_{n} \Omega_{Y}^{j} \simeq\left(\mathcal{E}_{n} \underset{\mathcal{O}_{Y_{n}}}{\stackrel{\mathrm{L}}{\otimes}} \mathcal{O}_{Y}\right) \stackrel{\stackrel{\mathrm{L}}{\otimes}}{\mathcal{O}_{Y}} \operatorname{gr}^{i} W_{n} \Omega_{Y}^{j}
$$

Because $\mathcal{E}_{n}$ and $\mathcal{O}_{Y_{n}}$ are flat relatively to $W_{n}$, we have

$$
\mathcal{E}_{n} \underset{\mathcal{O}_{Y_{n}}}{\stackrel{\mathrm{L}}{\otimes}} \mathcal{O}_{Y} \simeq \mathcal{E}_{n} \underset{\mathcal{O}_{Y_{n}}}{\stackrel{\mathrm{L}}{\otimes}}\left(\mathcal{O}_{Y_{n}} \underset{W_{n}}{\stackrel{\mathrm{L}}{\otimes}} k\right) \simeq \mathcal{E}_{n} \underset{W_{n}}{\stackrel{\mathrm{L}}{\otimes}} k \stackrel{\sim}{\longrightarrow} \mathcal{E}_{1},
$$

so it suffices to prove that

$$
\mathcal{T o r}_{q}^{\mathcal{O}_{Y}}\left(\mathcal{E}_{1}, \operatorname{gr}^{i} W_{n} \Omega_{Y}^{j}\right)=0
$$

for $q \geq 1$ and all $i, j$.

Since $\mathcal{E}_{1}$ is the evaluation on $Y$ of a crystal, its connection $\nabla_{1}$ is quasinilpotent [7, Def. 4.10]. Let $\mathcal{T}_{Y}=\Omega_{Y}^{1 \vee}$ be the sheaf of $k$-derivations on $Y$ and $\psi: \mathcal{T}_{Y} \rightarrow \mathcal{E} n d_{\mathcal{O}_{Y}}\left(\mathcal{E}_{1}\right)$ the $p$-curvature of $\nabla_{1}[15,5.0]$. We define an increasing filtration of $\mathcal{E}_{1}$ by horizontal submodules $\mathcal{E}_{1}^{m}, m \geq 0$, by setting, for any affine open subset $U \subset Y$,

(2.5.2) $\quad \Gamma\left(U, \mathcal{E}_{1}^{m}\right)=$

$$
\left\{x \in \Gamma(U, \mathcal{E}) \mid \forall D_{1}, \ldots, D_{m} \in \Gamma\left(U, \mathcal{T}_{Y}\right), \psi\left(D_{1}\right) \cdots \psi\left(D_{m}\right)(x)=0\right\}
$$

$[15,5.5]$. As $\nabla_{1}$ is quasi-nilpotent, this filtration is exhaustive, and it suffices to prove that, for $q \geq 1, m \geq 0$, and all $i, j$,

$$
\mathcal{T}_{0} r_{q}^{\mathcal{O}_{Y}}\left(\mathcal{E}_{1}^{m}, \operatorname{gr}^{i} W_{n} \Omega_{Y}^{j}\right)=0 .
$$

By construction, $\mathcal{E}_{1}^{0}=0$ and, for $m \geq 1$, each quotient $\mathcal{E}_{1}^{m} / \mathcal{E}_{1}^{m-1}$ has $p$-curvature 0 . Therefore, for all $m$, there is an $\mathcal{O}_{Y}$-module $\mathcal{F}^{m}$ such that $\mathcal{E}_{1}^{m} / \mathcal{E}_{1}^{m-1} \simeq F^{*} \mathcal{F}^{m}$. Since $F$ is flat, it is enough to prove that, for all $q \geq 1$, $m \geq 0$, and all $i, j$,

$$
\mathcal{T}_{0} r_{q}^{\mathcal{O}_{Y}}\left(\mathcal{F}^{m}, \operatorname{gr}^{i} W_{n} \Omega_{Y}^{j}\right)=0,
$$

$\operatorname{gr}^{i} W_{n} \Omega_{Y}^{j}$ being now viewed as an $\mathcal{O}_{Y}$-module thanks to the composition

$$
\mathcal{O}_{Y} \stackrel{F}{\longrightarrow} \mathcal{O}_{Y} \stackrel{\bar{t}_{F}}{\longrightarrow} W_{n} \mathcal{O}_{Y} / p W_{n} \mathcal{O}_{Y} .
$$

By Lemma 2.4, this homomorphism is the factorization

$$
\bar{F}: \mathcal{O}_{Y} \longrightarrow W_{n} \mathcal{O}_{Y} / p W_{n} \mathcal{O}_{Y}
$$

of the Frobenius action on $W_{n} \mathcal{O}_{Y} / p W_{n} \mathcal{O}_{Y} \cdot \operatorname{As~gr}^{i} W_{n} \Omega_{Y}^{j}$ is a locally free $\mathcal{O}_{Y^{-}}$ module for this structure [14, I, 3.9], the lemma follows. 
THEOREM 2.6. Let $Y$ be a smooth $k$-scheme. For some $n \geq 1$, let $\mathcal{E}$ be a crystal on $Y / W_{n}$, flat over $W_{n}$, and let $\mathcal{E}_{n}^{W}$ be the evaluation of $\mathcal{E}$ on the $P D$ thickening $\left(Y, W_{n} Y\right)$. Then

$$
\mathcal{T}_{o} r_{q}^{W_{n} \mathcal{O}_{Y}}\left(\mathcal{E}_{n}^{W}, \operatorname{Fil}^{i} W_{r} \Omega_{Y}^{j}\right)=\mathcal{T}_{o r}{ }_{q}^{W_{n} \mathcal{O}_{Y}}\left(\mathcal{E}_{n}^{W}, \operatorname{gr}^{i} W_{r} \Omega_{Y}^{j}\right)=0
$$

for $q \geq 1,1 \leq r \leq n$, and all $i, j$.

Proof. The statement is local on $Y$, hence we may assume that $Y$ is affine and has a smooth formal lifting $Y$ over $W$ endowed with a lifting $F$ of the absolute Frobenius endomorphism of $Y$. Let $Y_{n}$ be the reduction of $Y$ on $W_{n}$ and $\mathcal{E}_{n}$ the evaluation of $\mathcal{E}$ on $Y_{n}$. By (2.2.2), $t_{F}$ provides an isomorphism

$$
\mathcal{E}_{n} \otimes_{\mathcal{O}_{Y_{n}}} W_{n} \mathcal{O}_{Y} \stackrel{\sim}{\longrightarrow} \mathcal{E}_{n}^{W} .
$$

Applying (2.5.1) for $i=j=0$ and $r=n$, we obtain in $D^{-}\left(W_{n} \mathcal{O}_{Y}\right)$ an isomorphism

$$
\mathcal{E}_{n} \underset{\mathcal{O}_{Y_{n}}}{\stackrel{\mathrm{L}}{\otimes}} W_{n} \mathcal{O}_{Y} \stackrel{\sim}{\longrightarrow} \mathcal{E}_{n}^{W} .
$$

On the other hand, the transitivity isomorphism for the derived extension of scalars yields

$$
\begin{aligned}
& \mathcal{E}_{n} \underset{\mathcal{O}_{Y_{n}}}{\stackrel{\mathrm{L}}{\otimes}} \mathrm{Fil}^{i} W_{r} \Omega_{Y}^{j} \stackrel{\sim}{\longrightarrow}\left(\mathcal{E}_{n} \underset{\mathcal{O}_{Y_{n}}}{\stackrel{\mathrm{L}}{\otimes}} W_{n} \mathcal{O}_{Y}\right) \underset{W_{n} \mathcal{O}_{Y}}{\stackrel{\mathrm{L}}{\otimes}} \operatorname{Fil}^{i} W_{r} \Omega_{Y}^{j} \\
& \stackrel{\sim}{\longrightarrow} \mathcal{E}_{n}^{W} \underset{W_{n} \mathcal{O}_{Y}}{\stackrel{\mathrm{L}}{\otimes}} \mathrm{Fil}^{i} W_{r} \Omega_{Y}^{j} .
\end{aligned}
$$

By (2.5.1), the left hand side is acyclic in degrees $\neq 0$. The first vanishing of (2.6.1) follows, and the second one is obtained by the same argument.

We define the canonical filtration of $\mathcal{E}_{n}^{W} \otimes_{W_{n} \mathcal{O}_{Y}} W_{n} \Omega_{Y}^{j}$ by

$$
\begin{aligned}
& \operatorname{Fil}^{i}\left(\mathcal{E}_{n}^{W} \otimes_{W_{n} \mathcal{O}_{Y}} W_{n} \Omega_{Y}^{j}\right)= \\
& \qquad \begin{cases}\mathcal{E}_{n}^{W} \otimes_{W_{n} \mathcal{O}_{Y}} W_{n} \Omega_{Y}^{j} & \text { if } i \leq 0, \\
\operatorname{Ker}\left(\mathcal{E}_{n}^{W} \otimes_{W_{n} \mathcal{O}_{Y}} W_{n} \Omega_{Y}^{j} \rightarrow \mathcal{E}_{i}^{W} \otimes_{W_{i} \mathcal{O}_{Y}} W_{i} \Omega_{Y}^{j}\right) & \text { if } 1 \leq i \leq n, \\
0 & \text { if } i>n .\end{cases}
\end{aligned}
$$

Using (2.1.2), the theorem implies:

COROLLARY 2.7. Under the assumptions of the theorem, the natural map

$$
\mathcal{E}_{n}^{W} \otimes_{W_{n} \mathcal{O}_{Y}} \operatorname{Fil}^{i} W_{n} \Omega_{Y}^{j} \longrightarrow \operatorname{Fil}^{i}\left(\mathcal{E}_{n}^{W} \otimes_{W_{n} \mathcal{O}_{Y}} W_{n} \Omega_{Y}^{j}\right)
$$

is an isomorphism for all $i$. 
2.8. Let $Y$ be a smooth $k$-scheme, and assume that $n \geq 2$. If $\mathcal{E}$ is a crystal on $Y / W_{n}$, multiplication by $p$ on $\mathcal{E}_{n}^{W} \otimes W_{n} \Omega_{Y}^{j}$ vanishes on the image of $\mathcal{E}_{n}^{W} \otimes \mathrm{Fil}^{n-1} W_{n} \Omega_{Y}^{j}$. Factorizing and taking the isomorphism (2.1.2) into account, one gets a canonical homomorphism

$$
\underline{p}: \mathcal{E}_{n-1}^{W} \otimes_{W_{n-1} \mathcal{O}_{Y}} W_{n-1} \Omega_{Y}^{j} \rightarrow \mathcal{E}_{n}^{W} \otimes_{W_{n} \mathcal{O}_{Y}} W_{n} \Omega_{Y}^{j} .
$$

We recall that, when $\mathcal{E}=\mathcal{O}_{Y / W_{n}}$, the morphism $p$ is an injection

$$
\underline{p}: W_{n-1} \Omega_{Y}^{j} \hookrightarrow W_{n} \Omega_{Y}^{j}
$$

for all $j$ [14, Prop. 3.4]. It follows that, for $i \geq 1$, there is an exact sequence

$$
0 \rightarrow W_{n-1} \Omega_{Y}^{j} / p^{i-1} W_{n-1} \Omega_{Y}^{j} \stackrel{p}{\longrightarrow} W_{n} \Omega_{Y}^{j} / p^{i} W_{n} \Omega_{Y}^{j} \rightarrow W_{n} \Omega_{Y}^{j} / p W_{n} \Omega_{Y}^{j} \rightarrow 0 .
$$

We will show later that, when $\mathcal{E}$ is flat over $W_{n}$, the homomorphism (2.8.1) is still injective (see Corollary 2.11). This is a consequence of another Tor-independence property, which we prove next.

Lemma 2.9. Let $Y$ be a smooth $k$-scheme. For $n \geq 1$ and all $j$, the sheaf $W_{n} \Omega_{Y}^{j} / p W_{n} \Omega_{Y}^{j}$ is a locally free $\mathcal{O}_{Y}$-module of finite rank for the structure defined by the homomorphism $\bar{F}: \mathcal{O}_{Y} \rightarrow W_{n} \mathcal{O}_{Y} / p W_{n} \mathcal{O}_{Y}$ (2.4.1).

Proof. We proceed by induction on $n$, the claim being clear for $n=1$. We set $W_{n} \Omega_{Y}^{j}=0$ for $n=0$. For $n \geq 1$, the commutative diagram with exact rows

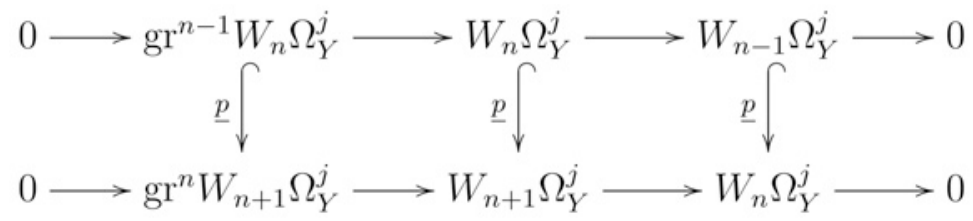

yields an exact sequence

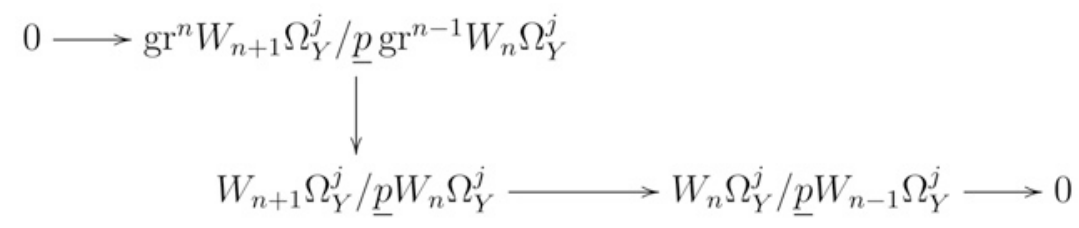

in which the morphisms are $\mathcal{O}_{Y}$-linear for the module structure defined by $\bar{F}$. As $\underline{p} W_{n-1} \Omega_{Y}^{j}=p W_{n} \Omega_{Y}^{j}$ for all $n \geq 1$, the induction hypothesis reduces to 
proving that $\mathrm{gr}^{n} W_{n+1} \Omega_{Y}^{j} / p \mathrm{gr}^{n-1} W_{n} \Omega_{Y}^{j}$ is a locally free finitely generated $\mathcal{O}_{Y}$-module for the structure defined by $\bar{F}$. By [14, I, (3.10.4)], there is a commutative diagram

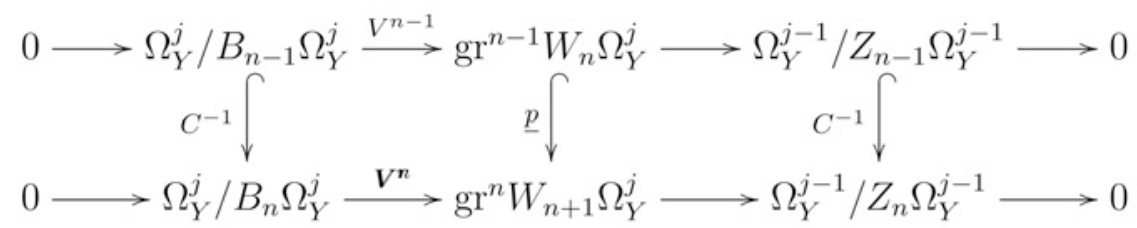

in which the exterior columns are defined by the inverse Cartier operator $C^{-1}$ and we set $B_{0} \Omega_{Y}^{j}=0, Z_{0} \Omega_{Y}^{j}=\Omega_{Y}^{j}$. All maps in this diagram become $\mathcal{O}_{Y}$-linear maps if we endow the terms of the middle column with the structure defined by $\bar{F}$, the exterior terms of the upper row with the structure defined by $F^{n}$, and the exterior terms of the lower row with the structure defined by $F^{n+1}$ (see [14, I, Cor. 3.9]). Therefore, the corresponding cokernel sequence, which can be written as

$$
0 \rightarrow B \Omega_{Y}^{j+1} \rightarrow \mathrm{gr}^{n} W_{n+1} \Omega_{Y}^{j} / \underline{p} \mathrm{gr}^{n-1} W_{n} \Omega_{Y}^{j} \rightarrow B \Omega_{Y}^{j} \rightarrow 0,
$$

is an $\mathcal{O}_{Y^{-}}$-linear exact sequence when $B \Omega_{Y}^{j}$ and $B \Omega_{Y}^{j+1}$ are viewed as $\mathcal{O}_{Y^{-}}$ modules thanks to $F^{n+1}$. By [14, 0, Prop. 2.2.8], they are then locally free of finite rank over $\mathcal{O}_{Y}$, which ends the proof.

THEOREM 2.10. Let $Y$ be a smooth $k$-scheme, and let $\mathcal{E}$ be a crystal on $Y / W_{n}$, flat over $W_{n}$. Let $\mathcal{E}_{n}^{W}$ be the evaluation of $\mathcal{E}$ on $\left(Y, W_{n} Y\right)$. Then

$$
\mathcal{T}_{0} r_{q}^{W_{n} \mathcal{O}_{Y}}\left(\mathcal{E}_{n}^{W}, W_{r} \Omega_{Y}^{j} / p^{i} W_{r} \Omega_{Y}^{j}\right)=\mathcal{T}_{0} r_{q}^{W_{n} \mathcal{O}_{Y}}\left(\mathcal{E}_{n}^{W}, p^{i} W_{r} \Omega_{Y}^{j}\right)=0
$$

for $q \geq 1,1 \leq r \leq n, i \geq 0$, and all $j$.

Proof. Thanks to Theorem 2.6, it suffices to prove the vanishing of the left hand side.

The statement is trivial for $i=0$. Let us prove it first for $i=1$. We may assume that $Y$ is affine, and we can choose a smooth formal scheme $Y$ lifting $Y$ over $W$, together with a lifting $F$ of the absolute Frobenius endomorphism of $Y$. Then the isomorphism (2.6.2) and the transitivity of the derived extension of scalars show as in the proof of Theorem 2.6 that it is equivalent to prove the relation

$$
\mathcal{T} r_{q}^{\mathcal{O}_{Y_{n}}}\left(\mathcal{E}_{n}, W_{r} \Omega_{Y}^{j} / p W_{r} \Omega_{Y}^{j}\right)=0,
$$

where $W_{r} \Omega_{Y}^{j} / p W_{r} \Omega_{Y}^{j}$ is viewed as an $\mathcal{O}_{Y_{n}}$-module thanks to $t_{F}$. As this 
module is annihilated by $p$, the flatness of $\mathcal{E}_{n}$ relative to $W_{n}$ implies as in the proof of Lemma 2.5 that it is equivalent to prove that

$$
\mathcal{T}^{\mathcal{O}_{q}^{\mathcal{O}_{Y}}}\left(\mathcal{E}_{1}, W_{r} \Omega_{Y}^{j} / p W_{r} \Omega_{Y}^{j}\right)=0
$$

where $\mathcal{E}_{1}=\mathcal{E}_{n} / p \mathcal{E}_{n}$ and $W_{r} \Omega_{Y}^{j} / p W_{r} \Omega_{Y}^{j}$ is viewed as an $\mathcal{O}_{Y}$-module thanks to the reduction $\bar{t}_{F}: \mathcal{O}_{Y} \rightarrow W_{r} \mathcal{O}_{Y} / p W_{r} \mathcal{O}_{Y}$ of $t_{F}$. Repeating again the proof of Lemma 2.5, we can use the $p$-curvature filtration of $\mathcal{E}_{1}$ to reduce (2.10.3) to the relation

$$
\mathcal{T} r_{q}^{\mathcal{O}_{Y}}\left(\mathcal{F}, W_{r} \Omega_{Y}^{j} / p W_{r} \Omega_{Y}^{j}\right)=0,
$$

where $\mathcal{F}$ is an $\mathcal{O}_{Y}$-module and $W_{r} \Omega_{Y}^{j} / p W_{r} \Omega_{Y}^{j}$ is viewed as an $\mathcal{O}_{Y}$-module through the composition $\bar{t}_{F} \circ F$. As the latter is equal to $\bar{F}$ by Lemma 2.4, relation (2.10.4) is then a consequence of Lemma 2.9.

This proves that, for $i=1, \mathcal{T}_{o} r_{q}^{W_{n} \mathcal{O}_{Y}}\left(\mathcal{E}_{n}^{W}, W_{r} \Omega_{Y}^{j} / p^{i} W_{r} \Omega_{Y}^{j}\right)$ vanishes for $q \geq 1,1 \leq r \leq n$, and all $j$. Moreover, by Theorem 2.6, it also vanishes when $r=1$ for $q \geq 1, i \geq 0$, and all $j$. Using the exact sequences (2.8.2) and the previous result for $i=1$, one can then argue by induction on $r$ to prove that the same vanishing holds for $1 \leq r \leq n$ and all $i \geq 0$.

COROLlary 2.11. Let $Y$ be a smooth $k$-scheme, and let $\mathcal{E}$ be a crystal on $Y / W_{n}$ for some $n \geq 2$. If $\mathcal{E}$ is flat over $W_{n}$, the homomorphism (2.8.1) is an injection

$$
\underline{p}: \mathcal{E}_{n-1}^{W} \otimes_{W_{n-1} \mathcal{O}_{Y}} W_{n-1} \Omega_{Y}^{j} \hookrightarrow \mathcal{E}_{n}^{W} \otimes_{W_{n} \mathcal{O}_{Y}} W_{n} \Omega_{Y}^{j} .
$$

Proof. Since $\underline{p}: W_{n-1} \Omega_{Y}^{j} \rightarrow W_{n} \Omega_{Y}^{j}$ is injective, it follows from the theorem that

$$
\mathrm{Id} \otimes \underline{p}: \mathcal{E}_{n}^{W} \otimes_{W_{n} \mathcal{O}_{Y}} W_{n-1} \Omega_{Y}^{j} \rightarrow \mathcal{E}_{n}^{W} \otimes{ }_{W_{n} \mathcal{O}_{Y}} W_{n} \Omega_{Y}^{j}
$$

is injective too. Using (2.1.2), we get an isomorphism

$$
\mathcal{E}_{n}^{W} \otimes_{W_{n} \mathcal{O}_{Y}} W_{n-1} \Omega_{Y}^{j} \stackrel{\sim}{\longrightarrow} \mathcal{E}_{n-1}^{W} \otimes_{W_{n-1} \mathcal{O}_{Y}} W_{n-1} \Omega_{Y}^{j},
$$

which identifies Id $\otimes p$ to the homomorphism $p$ defined by (2.8.1) and completes the proof.

2.12. We now assume that $\mathcal{E}$ is a crystal on $Y / W$, flat over $W$, and we consider the inverse system of $W_{n} \mathcal{O}_{Y}$-modules $\mathcal{E}_{n}^{W}$ defined in 2.1 by taking the evaluation of $\mathcal{E}$ at all thickenings $\left(Y, W_{n} Y\right)$ when $n$ varies. We also assume that $\mathcal{E}$ is a quasi-coherent crystal, i.e., that, for any PD-thickening 
$(U, T)$ in $\operatorname{Crys}(Y / W)$, the evaluation $\mathcal{E}_{T}$ of $\mathcal{E}$ on $T$ is a quasi-coherent $\mathcal{O}_{T^{-}}$ module. It is equivalent to ask that, for any $n \geq 1$, this condition be verified for thickenings of the form $\left(U_{\alpha}, U_{\alpha, n}\right)$, where $U_{\alpha}$ varies in an open covering of $Y$ and $U_{\alpha, n}$ is a smooth lifting of $U_{\alpha}$ on $W_{n}$. Thanks to [14, I, Prop. 1.13.1], the $W_{n} \mathcal{O}_{Y}$-module $\mathcal{E}_{n}^{W} \otimes_{W_{n} \mathcal{O}_{Y}} W_{n} \Omega_{Y}^{j}$ is then quasi-coherent for all $n \geq 1$ and all $j$.

For all $j$, we define

$$
\widehat{\mathcal{E}}^{W} \widehat{\otimes}_{W \mathcal{O}_{Y}} W \Omega_{Y}^{j}:=\lim _{\overleftarrow{n}}\left(\mathcal{E}_{n}^{W} \otimes_{W_{n} \mathcal{O}_{Y}} W_{n} \Omega_{Y}^{j}\right)
$$

By construction, we have projections

$$
\widehat{\mathcal{E}}^{W} \widehat{\otimes}_{W \mathcal{O}_{Y}} W \Omega_{Y}^{j} \rightarrow \mathcal{E}_{i}^{W} \otimes_{W_{i} \mathcal{O}_{Y}} W_{i} \Omega_{Y}^{j}
$$

for each $i \geq 1$, and, since the inverse system has surjective transition maps and quasi-coherent terms, the Mittag-Leffler criterion implies that these projections are surjective. We define the canonical filtration of $\widehat{\mathcal{E}}^{W} \widehat{\otimes}_{W \mathcal{O}_{Y}} W \Omega_{Y}^{j}$ by

$\operatorname{Fil}^{i}\left(\widehat{\mathcal{E}}^{W} \widehat{\otimes}_{W \mathcal{O}_{Y}} W \Omega_{Y}^{j}\right)= \begin{cases}\widehat{\mathcal{E}} \widehat{\otimes}_{W \mathcal{O}_{Y}} W \Omega_{Y}^{j} & \text { if } i \leq 0, \\ \operatorname{Ker}\left(\widehat{\mathcal{E}}^{W} \widehat{\otimes}_{W \mathcal{O}_{Y}} W \Omega_{Y}^{j} \rightarrow \mathcal{E}_{i}^{W} \otimes_{W_{i} \mathcal{O}_{Y}} W_{i} \Omega_{Y}^{j}\right) & \text { if } i \geq 1 .\end{cases}$

Note that, for $i \geq 1$,

$$
\operatorname{Fil}^{i}\left(\widehat{\mathcal{E}}^{W} \widehat{\otimes}_{W \mathcal{O}_{Y}} W \Omega_{Y}^{j}\right) \stackrel{\sim}{\longrightarrow} \lim _{\overleftarrow{n}} \operatorname{Ker}\left(\mathcal{E}_{n}^{W} \otimes{ }_{W_{n} \mathcal{O}_{Y}} W_{n} \Omega_{Y}^{j} \rightarrow \mathcal{E}_{i}^{W} \otimes W_{i} \mathcal{O}_{Y} W_{i} \Omega_{Y}^{j}\right),
$$

hence the Mittage-Leffler criterion implies that, for any affine open subset $U \subset Y$,

$$
H^{q}\left(U, \operatorname{Fil}^{i}\left(\widehat{\mathcal{E}}^{W} \widehat{\otimes}_{W \mathcal{O}_{Y}} W \Omega_{Y}^{j}\right)\right)=0
$$

for $q \geq 1$ and all $i, j$.

For each affine open subset $U \subset Y$, the canonical filtration endows $\Gamma\left(U, \widehat{\mathcal{E}}^{W} \widehat{\otimes}_{W \mathcal{O}_{Y}} W \Omega_{Y}^{j}\right)$ with a topology, which will be called the canonical topology. From (2.12.1), we deduce the isomorphism

(2.12.2) $\Gamma\left(U, \widehat{\mathcal{E}}^{W} \widehat{\otimes}_{W \mathcal{O}_{Y}} W \Omega_{Y}^{j}\right) / \mathrm{Fil}^{i} \Gamma\left(U, \widehat{\mathcal{E}}^{W} \widehat{\otimes}_{W \mathcal{O}_{Y}} W \Omega_{Y}^{j}\right) \stackrel{\sim}{\longrightarrow} \Gamma\left(U, \mathcal{E}_{i}^{W} \otimes_{W_{i} \mathcal{O}_{Y}} W_{i} \Omega_{Y}^{j}\right)$

for $i \geq 1$. It follows that $\Gamma\left(U, \widehat{\mathcal{E}}^{W} \widehat{\otimes}_{W \mathcal{O}_{Y}} W \Omega_{Y}^{j}\right)$ is separated and complete for the canonical topology.

Proposition 2.13. Let $Y$ be a smooth $k$-scheme, and let $\mathcal{E}$ be a crystal on $Y / W$. Assume that $\mathcal{E}$ is flat over $W$ and quasi-coherent. 
(i) For all $j$, multiplication by $p$ is injective on $\widehat{\mathcal{E}}^{W} \widehat{\otimes}_{W \mathcal{O}_{Y}} W \Omega_{Y}^{j}$.

(ii) If $U \subset Y$ is an affine open subset, then, for all $i \geq 0$ and all $j$, $p^{i} \Gamma\left(U, \widehat{\mathcal{E}}^{W} \widehat{\otimes}_{W \mathcal{O}_{Y}} W \Omega_{Y}^{j}\right)$ is closed in $\Gamma\left(U, \widehat{\mathcal{E}}^{W} \widehat{\otimes}_{W \mathcal{O}_{Y}} W \Omega_{Y}^{j}\right)$ for the canonical topology, and $\Gamma\left(U, \widehat{\mathcal{E}}^{W} \widehat{\otimes}_{W \mathcal{O}_{Y}} W \Omega_{Y}^{j}\right)$ is separated and complete for the $p$ adic topology.

Proof. As multiplication by $p$ on $\widehat{\mathcal{E}}^{W} \widehat{\otimes}_{W \mathcal{O}_{Y}} W \Omega_{Y}^{j}$ is the inverse limit of the maps $p$ defined in (2.8.1), assertion (i) results from Corollary 2.11.

Let $U$ be an affine open subset of $Y$, and let $i \in \mathbb{N}$. Using assertion (i) and Corollary 2.11, we can write a commutative diagram with exact columns

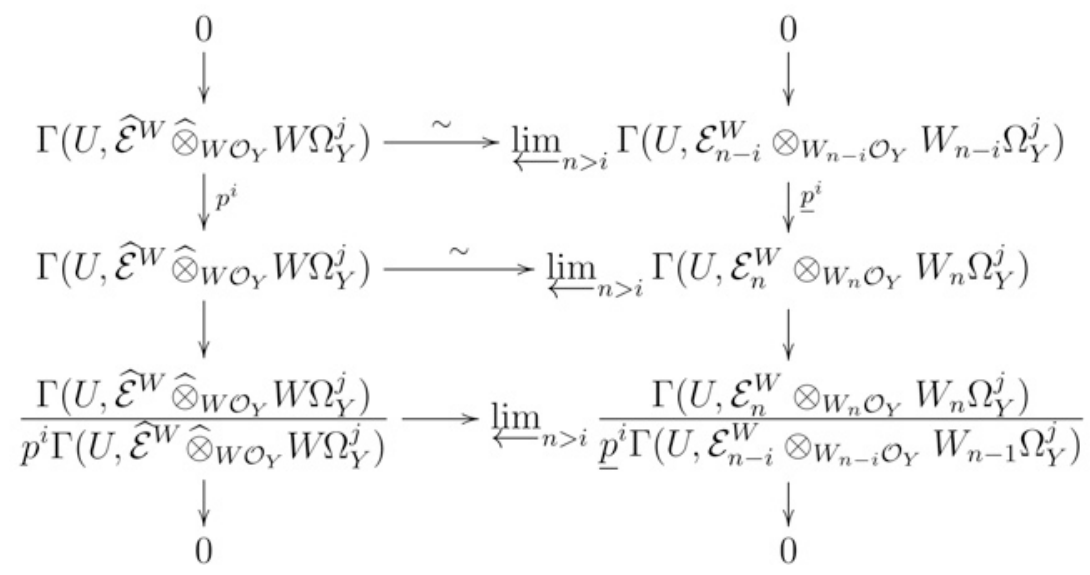

in which the surjectivity in the right hand side column results from the surjectivity of the transition maps in the inverse system $\left(\Gamma\left(U, \mathcal{E}_{n-i}^{W} \otimes_{W_{n-i} \mathcal{O}_{Y}} W_{n-i} \Omega_{Y}^{j}\right)\right)_{n>i}$. It follows that the bottom horizontal arrow is an isomorphism. The quasi-coherence assumption on $\mathcal{E}$ implies that

$$
\left.\underline{p}^{i} \Gamma\left(U, \mathcal{E}_{n-i}^{W} \otimes W_{n-i} \mathcal{O}_{Y} W_{n-1} \Omega_{Y}^{j}\right)\right)=p^{i} \Gamma\left(U, \mathcal{E}_{n}^{W} \otimes_{W_{n} \mathcal{O}_{Y}} W_{n} \Omega_{Y}^{j}\right)
$$

and that the map (2.12.2)

$$
\Gamma\left(U, \widehat{\mathcal{E}}^{W} \widehat{\otimes}_{W \mathcal{O}_{Y}} W \Omega_{Y}^{j}\right) \rightarrow \Gamma\left(U, \mathcal{E}_{n}^{W} \otimes W_{n} \mathcal{O}_{Y} W_{n} \Omega_{Y}^{j}\right)
$$

is surjective. Therefore the quotient in the right hand side column can be rewritten as

$$
\lim _{n>i} \frac{\Gamma\left(U, \widehat{\mathcal{E}}^{W} \widehat{\otimes}_{W \mathcal{O}_{Y}} W \Omega_{Y}^{j}\right)}{p^{i} \Gamma\left(U, \widehat{\mathcal{E}}^{W} \widehat{\otimes}_{W \mathcal{O}_{Y}} W \Omega_{Y}^{j}\right)+\mathrm{Fil}^{n} \Gamma\left(U, \widehat{\mathcal{E}}^{W} \widehat{\otimes}_{W \mathcal{O}_{Y}} W \Omega_{Y}^{j}\right)} .
$$


This proves the first part of assertion (ii). As $\Gamma\left(U, \widehat{\mathcal{E}}^{W} \widehat{\otimes}_{W \mathcal{O}_{Y}} W \Omega_{Y}^{j}\right)$ is separated and complete for the canonical topology, which is coarser than the $p$-adic topology, the second part of assertion (ii) follows by [9, Ch. III, $\S 3, \mathrm{n}^{0}$ 5, Cor. 2 to Prop. 10].

\section{De Rham-Witt cohomology with coefficients}

In this section, we extend the classical comparison theorem between the crystalline and de Rham-Witt cohomologies of a smooth $k$-scheme $Y$ to the case of cohomologies with coefficients in a crystal on $Y / W_{n}$ that is flat over $W_{n}$.

As indicated in our general conventions, we now assume until the end of the article that all schemes are quasi-compact and separated. We first recall the construction of the comparison morphism in the case of constant coefficients [14, II, 1], starting at the level of complexes of Zariski sheaves on $Y$.

3.1. Let $\mathbb{P}$ be a smooth formal scheme over $W$, with reduction $P_{n}$ over $W_{n}$ and special fibre $P=P_{1}$. Let $X \subset P$ be a closed subscheme. We denote by $\mathcal{J}_{n}$ (resp. $\mathcal{J}$ ) the ideal of $X$ in $P_{n}$ (resp. $\mathbb{P}$ ) and by $\mathcal{P}_{n}$ (resp. $\widehat{\mathcal{P}}$ ) the divided power envelope of $\mathcal{J}_{n}$ (resp. the $p$-adic completion of the divided power envelope of $\mathcal{J}$ ). In these constructions, we impose that all divided powers be compatible with the natural divided powers of $p$, which implies that $\mathcal{P}_{n} \simeq \mathcal{P}_{n+1} / p^{n} \mathcal{P}_{n+1}$ for all $n$ and $\widehat{\mathcal{P}}=\lim _{\overleftarrow{n}} \mathcal{P}_{n}$. Divided power envelopes have a natural connection, which allows to define the de Rham complexes $\mathcal{P}_{n} \otimes \Omega_{P_{n}}^{\bullet}$ and $\widehat{\mathcal{P}} \otimes \Omega_{\mathrm{P}}^{\bullet}$; these complexes are supported in $X$. Let $u_{X / W_{n}}$ (resp. $u_{X / W}$ ) be the projection from the crystalline topos of $X$ relative to $W_{n}$ (resp. $W$ ) to the Zariski topos of $X$. In its local form, the comparison theorem between crystalline and de Rham cohomologies [7, (7.1.2)] provides functorial isomorphisms

$$
\begin{aligned}
\mathbb{R} u_{X / W_{n} *} \mathcal{O}_{X / W_{n}} & \simeq \mathcal{P}_{n} \otimes \Omega_{P_{n} / W_{n}}^{\bullet}, \\
\mathbb{R} u_{X / W *} \mathcal{O}_{X / W} & \simeq \widehat{\mathcal{P}} \otimes \Omega_{\mathbb{P} / W}^{\bullet} .
\end{aligned}
$$

in $D^{\mathrm{b}}\left(X, W_{n}\right)$ and $D^{\mathrm{b}}(X, W)$ (note that the tensor product does not need to be completed, since $\Omega_{\mathrm{P} / W}^{\bullet}$ is a locally free finitely generated $\mathcal{O}_{\mathrm{P}}$-module). Taking sections on $X$, one gets in $D^{\mathrm{b}}\left(W_{n}\right)$ and $D^{\mathrm{b}}(W)$ the global comparison isomorphisms

$$
\begin{aligned}
\mathbb{R} \Gamma_{\text {crys }}\left(X / W_{n}, \mathcal{O}_{X / W_{n}}\right) & \simeq \mathbb{R} \Gamma\left(X, \mathcal{P}_{n} \otimes \Omega_{P_{n} / W_{n}}^{\bullet}\right), \\
\mathbb{R} \Gamma_{\text {crys }}\left(X / W, \mathcal{O}_{X / W}\right) & \simeq \mathbb{R} \Gamma\left(X, \widehat{\mathcal{P}} \otimes \Omega_{\mathrm{P} / W}^{\bullet}\right) .
\end{aligned}
$$


These isomorphisms can be generalized to the case where the datum of the embedding $X \hookrightarrow \mathbb{P}$ is replaced by the data of an affine open covering $\mathfrak{U}=\left(U_{\alpha}\right)$ of $X$ and of closed embeddings $U_{\alpha} \hookrightarrow \mathbb{P}_{\alpha}$ into smooth formal schemes $[14,0,3.2 .6]$. For each multi-index $\underline{\alpha}=\left(\alpha_{0}, \ldots, \alpha_{i}\right)$, let $U_{\underline{\alpha}}=U_{\alpha_{0}} \cap \cdots \cap U_{\alpha_{i}}, \mathbb{P}_{\underline{\alpha}}=\mathbb{P}_{\alpha_{0}} \times_{W} \cdots \times_{W} \mathbb{P}_{\alpha_{i}}$, let $\mathcal{J}_{\underline{\alpha}} \subset \mathcal{O}_{\mathbb{P}_{\alpha}}$ be the ideal defining the diagonal embeddings $U_{\alpha} \hookrightarrow \mathbb{P}_{\alpha}, \widehat{\mathcal{P}}_{\alpha}$ its completed divided power envelope, $P_{\underline{\alpha}, n}, \mathcal{J}_{\underline{\alpha}, n}, \mathcal{P}_{\underline{\alpha}, n}$ the reductions mod $p^{n}$ of $\mathbb{P}_{\underline{\alpha}}, \mathcal{J}_{\underline{\alpha}}, \mathcal{P}_{\underline{\alpha}}$, and let $j_{\alpha}: U_{\alpha} \hookrightarrow X$ be the inclusion. One can define Čech double complexes $j_{\bullet *}\left(\mathcal{P}_{\bullet}, n \otimes \Omega_{P_{\bullet}, n}^{\bullet}\right)\left(\right.$ resp. $\left.j_{\bullet *}\left(\widehat{\mathcal{P}}_{\bullet} \otimes \Omega_{\mathrm{P}_{\bullet}}^{\bullet}\right)\right)$ with general term

$$
\prod_{\underline{\alpha}=\left(\alpha_{0}, \ldots, \alpha_{i}\right)} j_{\underline{\alpha} *}\left(\mathcal{P}_{\underline{\alpha}, n} \otimes \Omega_{P_{\underline{\alpha}, n}}^{j}\right) \quad\left(\text { resp. } \quad \prod_{\underline{\alpha}=\left(\alpha_{0}, \ldots, \alpha_{i}\right)} j_{\underline{\alpha} *}\left(\widehat{\mathcal{P}}_{\underline{\alpha}} \otimes \Omega_{\mathbb{P}_{\underline{\alpha}}}^{j}\right)\right)
$$

in bidegree $(j, i)$. If one uses the subscript " $\mathrm{t}$ " to denote the total complex associated to a multicomplex, one can then generalize the isomorphisms (3.1.1) and (3.1.2) as

$$
\begin{aligned}
\mathbb{R} u_{X / W_{n} *} \mathcal{O}_{X / W_{n}} & \simeq\left(j _ { \bullet * } \left(\mathcal{P}_{\bullet}, n\right.\right. \\
\mathbb{R} u_{X / W *} \mathcal{O}_{X / W} & \simeq\left(j_{\bullet *}\left(\widehat{\mathcal{P}} \bullet \Omega_{P_{\bullet}, n}^{\bullet}\right)\right)_{\mathrm{t}}, \\
\mathrm{P}_{\bullet} & ))_{\mathrm{t}},
\end{aligned}
$$

and one gets similar generalizations of (3.1.3) and (3.1.4).

Definition 3.2. Let $\mathbb{P}$ be a smooth formal scheme over $W$, endowed with a $\sigma$-semilinear morphism $F: \mathbb{P} \rightarrow \mathbb{P}$ lifting the absolute Frobenius endomorphism of its special fibre $P$, and let $X \subset P$ be a closed subscheme. For any $n \geq 1$, let $t_{F}: \mathcal{O}_{P_{n}} \rightarrow W_{n} \mathcal{O}_{P}$ be the homomorphism (2.2.1) defined by $F$. Then the composition

$$
\mathcal{O}_{P_{n}} \stackrel{t_{F}}{\longrightarrow} W_{n} \mathcal{O}_{P} \rightarrow W_{n} \mathcal{O}_{X}
$$

maps $\mathcal{J}_{n}$ to $V W_{n-1} \mathcal{O}_{X} \subset W_{n} \mathcal{O}_{X}$. Using the natural divided powers of the ideal $V W_{n-1} \mathcal{O}_{X}$ (which are compatible with the divided powers of $p$ ), the universal property of divided power envelopes provides a unique factorization of this composition through a homomorphism denoted

$$
h_{F}: \mathcal{P}_{n} \rightarrow W_{n} \mathcal{O}_{X},
$$

which commutes with the divided powers.

3.3. Let $Y$ be a smooth $k$-scheme, embedded through a closed immersion $Y \hookrightarrow P$ into the special fibre of a smooth formal scheme $\mathbb{P}$ over $W$. Assume that $\mathbb{P}$ is endowed with a Frobenius lifting $F$ as in 3.2, and keep for $Y$ the notation introduced for $X$ in 3.1-3.2. Then the homomorphism (3.2.1) 
extends as a morphism of complexes

$$
\Omega_{P_{n}}^{\bullet} \rightarrow W_{n} \Omega_{P}^{\bullet} \rightarrow W_{n} \Omega_{Y}^{\bullet} .
$$

Thanks to the structure of graded $\mathcal{P}_{n}$-algebra defined by $h_{F}$ on $W_{n} \Omega_{Y}^{\circ}$, this morphism defines by extension of scalars from $\mathcal{O}_{P_{n}}$ to $\mathcal{P}_{n}$ an $h_{F}$-semi-linear morphism

$$
\mathcal{P}_{n} \otimes \Omega_{P_{n}}^{\bullet} \rightarrow W_{n} \Omega_{Y}^{\bullet},
$$

which is still a morphism of complexes. When $n$ varies, these morphisms are compatible, and their inverse limit gives a morphism of complexes

$$
\widehat{\mathcal{P}} \otimes \Omega_{\mathrm{P}}^{\bullet} \rightarrow W \Omega_{Y}^{\bullet} .
$$

The morphisms (3.3.2) and (3.3.3) are functorial with respect to the triple $(Y, \mathbb{P}, F)$ in the following sense. Let $\mathbb{P}^{\prime}$ be a second smooth formal $W$ scheme, with special fibre $P^{\prime}$, let $Y^{\prime} \subset P^{\prime}$ be a smooth closed subscheme, and let $F^{\prime}: \mathbb{P}^{\prime} \rightarrow \mathbb{P}^{\prime}$ be a lifting of the Frobenius endomorphism of $P^{\prime}$. If $u: \mathbb{P}^{\prime} \rightarrow \mathbb{P}$ is a $W$-morphism commuting with $F$ and $F^{\prime}$, and inducing a $k$ morphism $f: Y^{\prime} \rightarrow Y$, then we get commutative diagrams

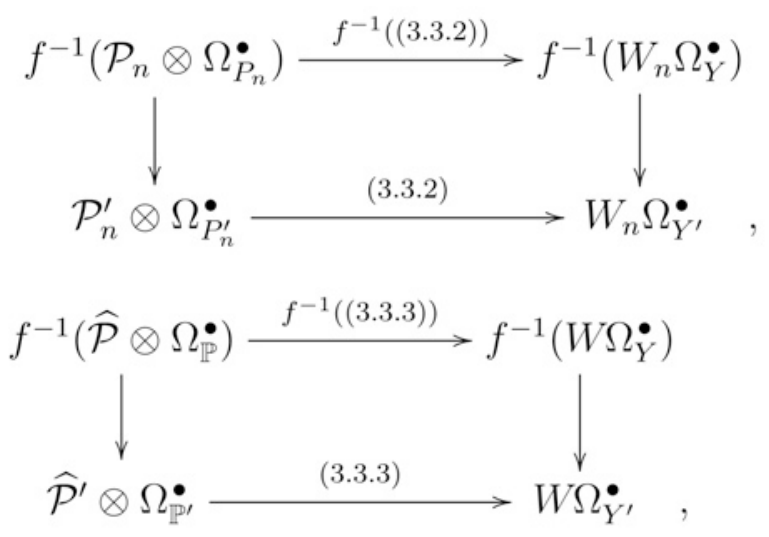

where the vertical maps are the functoriality morphisms.

The morphisms (3.3.2) and (3.3.3) are quasi-isomorphisms, and, via (3.1.1) and (3.1.2), they define the comparison isomorphism between crystalline and de Rham-Witt cohomologies [14, II, 1.4]. In the derived category $D^{\mathrm{b}}\left(Y, W_{n}\right)$ (resp. $\left.D^{\mathrm{b}}(Y, W)\right)$ of sheaves of $W_{n}$-modules (resp. $W$-modules) on $Y$, this comparison isomorphism does not depend on the choice of $(\mathbb{P}, F)$ (by the standard argument comparing two embeddings $Y \hookrightarrow(\mathbb{P}, F)$ and $Y \hookrightarrow\left(\mathbb{P}^{\prime}, F^{\prime}\right)$ to the diagonal embedding into $\left(\mathbb{P} \times_{W} \mathbb{P}^{\prime}, F \times F^{\prime}\right)$ via the projection maps). 
If $Y$ is quasi-projective, one can always find such an embedding ( $\mathbb{P}, F)$, since it suffices to choose for $\mathrm{P}$ an open subscheme of a projective space, endowed with the morphism induced by some lifting of the Frobenius endomorphism of the projective space. In the general case, one can choose an affine open covering $\mathfrak{U}=\left(U_{\alpha}\right)$ of $Y$ and closed immersions $U_{\alpha} \hookrightarrow \mathbb{P}_{\alpha}$ into smooth formal schemes endowed with a lifting of Frobenius. As in 3.1, one can then identify $\mathbb{R} u_{Y / W_{n} *} \mathcal{O}_{Y / W_{n}}$ with the total complex associated to the Cech double complex $j_{\bullet *}\left(\mathcal{P}_{\bullet, n} \otimes \Omega_{P_{\bullet}, n}^{\bullet}\right)\left(\operatorname{resp} . \mathbb{R} u_{Y / W *} \mathcal{O}_{Y / W}, j_{\bullet *}\left(\widehat{\mathcal{P}}_{\bullet} \otimes \Omega_{\mathrm{P}_{\bullet}}^{\bullet}\right)\right)$. On the other hand, the total complex associated to the Cech double complex $\check{C}^{\bullet}\left(\mathfrak{H}, W_{n} \Omega_{Y}^{\bullet}\right)$ (resp. $\left.\check{C}^{\bullet}\left(\mathfrak{H}, W \Omega_{Y}^{\bullet}\right)\right)$ provides a resolution of $W_{n} \Omega_{Y}^{\bullet}$ (resp. $W \Omega_{Y}^{\bullet}$ ). Endowing each $\mathbb{P}_{\underline{\alpha}}$ with the product $F_{\alpha_{0}} \times \cdots \times F_{\alpha_{i}}$ and using the functoriality (3.3.4) (resp. (3.3.5)), one gets between these double complexes a morphism that is defined on each intersection $U_{\underline{\alpha}}$ by the corresponding morphism (3.3.2) (resp. (3.3.3)). Since each of these is a quasi-isomorphism, so is the morphism induced between the associated total complexes. In the derived categories $D\left(Y, W_{n}\right)$ and $D(Y, W)$, this provides isomorphisms

$$
\begin{aligned}
\mathbb{R} u_{Y / W_{n} *} \mathcal{O}_{Y / W_{n}} & \stackrel{\sim}{\longrightarrow} W_{n} \Omega_{Y}^{\bullet}, \\
\mathbb{R} u_{Y / W *} \mathcal{O}_{Y / W} & \stackrel{\sim}{\longrightarrow} \Omega_{Y}^{\bullet},
\end{aligned}
$$

which extend the comparison isomorphisms (3.3.2) and (3.3.3) to the general case and do not depend on the choices (see [14, II, 1.1] for details).

3.4. Let us keep the hypothesis of the existence of an embedding $Y \hookrightarrow \mathbb{P}$ of $Y$ into a smooth formal scheme, and let $\mathcal{E}$ be a crystal on $Y / W_{n}$ for some $n \geq 1$ (resp. a crystal on $Y / W$ ).

We now denote by $\mathcal{E}_{n}$ the evaluation of $\mathcal{E}$ on the thickening $\left(Y, \operatorname{Spec}\left(\mathcal{P}_{n}\right)\right)$. By construction, $\mathcal{E}_{n}$ is a $\mathcal{P}_{n}$-module, and, by restriction of scalars, it can also be viewed as an $\mathcal{O}_{P_{n}}$-module. As such, it inherits from the crystal structure of $\mathcal{E}$ an integrable connection $\nabla_{n}: \mathcal{E}_{n} \rightarrow \mathcal{E}_{n} \otimes \Omega_{P_{n} / W_{n}}^{1}$, compatible with the natural connection on $\mathcal{P}_{n}$. This connection allows to define the de Rham complex $\mathcal{E}_{n} \otimes \Omega_{P_{n} / W_{n}}^{\bullet}$. When $\mathcal{E}$ is a crystal on $Y / W$, it can be viewed as a compatible family of crystals on $Y / W_{n}$ for all $n$. Thus, $\mathcal{E}$ defines for all $n$ a $\mathcal{P}_{n}$-module $\mathcal{E}_{n}$ endowed with an integrable connection $\nabla_{n}$, such that $\mathcal{E}_{n} \simeq \mathcal{E}_{n+1} / p^{n} \mathcal{E}_{n+1}$ as a module with connection. Then the $\widehat{\mathcal{P}}$-module $\widehat{\mathcal{E}}=\lim _{\overleftarrow{n}} \mathcal{E}_{n}$ has a connection $\nabla=\lim _{\overleftarrow{n}} \nabla_{n}$, and we get similarly the de Rham complex $\widehat{\mathcal{E}} \otimes \Omega_{\mathrm{P} / W}^{\bullet}$. We recall that the datum of $\left(\mathcal{E}_{n}, \nabla_{n}\right)(\operatorname{resp} .(\widehat{\mathcal{E}}, \nabla))$ is equivalent to the datum of the crystal $\mathcal{E}$. 
As in the case of cohomology with constant coefficients, the comparison theorem between crystalline and de Rham cohomologies yields functorial isomorphisms [7, Th. 7.1]

$$
\begin{aligned}
\mathbb{R} u_{Y / W_{n} *} \mathcal{E} & \simeq \mathcal{E}_{n} \otimes \Omega_{P_{n} / W_{n}}^{\bullet}, \\
\mathbb{R} \Gamma_{\text {crys }}\left(Y / W_{n}, \mathcal{E}\right) & \simeq \mathbb{R} \Gamma\left(Y, \mathcal{E}_{n} \otimes \Omega_{P_{n} / W_{n}}^{\bullet}\right),
\end{aligned}
$$

and, when $\mathcal{E}$ is a quasi-coherent crystal on $Y / W$ [7, Th. 7.23],

$$
\begin{gathered}
\mathbb{R} u_{Y / W *} \mathcal{E} \simeq \widehat{\mathcal{E}} \otimes \Omega_{\mathrm{P} / W}^{\bullet}, \\
\mathbb{R} \Gamma_{\text {crys }}(Y / W, \mathcal{E}) \simeq \mathbb{R} \Gamma\left(Y, \widehat{\mathcal{E}} \otimes \Omega_{\mathrm{P} / W}^{\bullet}\right) .
\end{gathered}
$$

If $\mathfrak{U}=\left(U_{\alpha}\right)$ is an affine open covering of $Y$ and $\left(U_{\alpha} \hookrightarrow \mathbb{P}_{\alpha}\right)$ a family of closed immersions into smooth formal schemes over $W$, these isomorphisms can be generalized when $\mathcal{E}$ is quasi-coherent by replacing $\mathcal{E}_{n} \otimes \Omega_{P_{n} / W_{n}}^{\bullet}$ and $\widehat{\mathcal{E}} \otimes \Omega_{\mathrm{P} / W}^{\bullet}$ with the total complexes $\left(j_{\bullet *}\left(\mathcal{E}_{P_{\bullet}, n} \otimes \Omega_{P_{\bullet}, n}^{\bullet} / W_{n}\right)\right)_{\mathrm{t}}$ and $\left(j_{\bullet *}\left(\widehat{\mathcal{E}}_{\mathrm{P}_{\bullet}} \otimes \Omega_{\mathrm{P}_{\bullet} / W}^{\bullet}\right)\right)_{\mathrm{t}}$ defined as in 3.1. When $\mathcal{E}$ is a non necessarily quasi-coherent crystal relative to $W_{n}$, a similar expression can be given using cohomological descent [14, 0, 3.2.6].

3.5. We keep the notation $\mathcal{E}_{n}^{W}$ used in 2.1 for the evaluation of $\mathcal{E}$ on the thickening $\left(Y, W_{n} Y\right)$. As shown by Étesse [11, II, 2], the crystal structure of $\mathcal{E}$ also provides a differential $\nabla_{n}^{W}: \mathcal{E}_{n}^{W} \rightarrow \mathcal{E}_{n}^{W} \otimes_{W_{n} \mathcal{O}_{Y}} W_{n} \Omega_{Y}^{1}$, which extends so as to define a complex $\mathcal{E}_{n}^{W} \otimes_{W_{n} \mathcal{O}_{Y}} W_{n} \Omega_{Y}^{\circ}$.

Let us assume again that $\mathbb{P}$ is endowed with a lifting $F$ of the absolute Frobenius endomorphism of $P$. Then the PD-morphism $h_{F}: \mathcal{P}_{n} \rightarrow W_{n} \mathcal{O}_{Y}$ defined in (3.2.2) corresponds to a morphism of thickenings $W_{n} Y \rightarrow \operatorname{Spec}\left(\mathcal{P}_{n}\right)$, and the crystal structure of $\mathcal{E}$ provides a natural $h_{F}$-semi-linear map

$$
h_{\mathcal{E}, F}: \mathcal{E}_{n} \longrightarrow \mathcal{E}_{n}^{W},
$$

the linear factorization of which gives an isomorphism

$$
1 \otimes h_{\mathcal{E}, F}: W_{n} \mathcal{O}_{Y} \otimes_{\mathcal{P}_{n}} \mathcal{E}_{n} \stackrel{\sim}{\longrightarrow} \mathcal{E}_{n}^{W}
$$

(generalizing (2.2.2), where $Y=P$ ). We can then take the tensor product over $h_{F}: \mathcal{P}_{n} \rightarrow W_{n} \mathcal{O}_{Y}$ of the morphism $h_{\mathcal{E}, F}$ with the semi-linear morphism of graded modules $\mathcal{P}_{n} \otimes \Omega_{P_{n}}^{\bullet} \rightarrow W_{n} \Omega_{Y}^{\bullet}$ defined in (3.3.2), and this defines an $h_{F}$-semi-linear morphism of graded modules

$$
\mathcal{E}_{n} \otimes_{\mathcal{O}_{P_{n}}} \Omega_{P_{n}}^{\bullet} \stackrel{\sim}{\longrightarrow} \mathcal{E}_{n} \otimes_{\mathcal{P}_{n}}\left(\mathcal{P}_{n} \otimes_{\mathcal{O}_{P_{n}}} \Omega_{P_{n}}^{\bullet}\right) \longrightarrow \mathcal{E}_{n}^{W} \otimes_{W_{n} \mathcal{O}_{Y}} W_{n} \Omega_{Y}^{\bullet} .
$$

It is easy to deduce from the constructions of $\nabla_{n}$ and $\nabla_{n}^{W}$ that this morphism is actually a morphism of complexes. When $\mathcal{E}$ is a crystal on $Y / W$, 
the inverse limit of these morphisms when $n$ varies defines a morphism

$$
\widehat{\mathcal{E}} \otimes_{\mathcal{O}_{\mathrm{P}}} \Omega_{\mathrm{P}}^{\bullet} \longrightarrow \widehat{\mathcal{E}}^{W} \widehat{\otimes}_{W \mathcal{O}_{Y}} W \Omega_{Y}^{\bullet},
$$

where the completed tensor product is defined as in 2.12 by

$$
\widehat{\mathcal{E}}^{W} \widehat{\otimes}_{W \mathcal{O}_{Y}} W \Omega_{Y}^{\bullet}=\lim _{\overleftarrow{n}}\left(\mathcal{E}_{n}^{W} \otimes_{W_{n} \mathcal{O}_{Y}} W_{n} \Omega_{Y}^{\bullet}\right)
$$

As in the constant case, these morphisms are functorial with respect to $(Y, P, F)$. Keeping the same notation and hypotheses than in 3.3, let $\mathcal{E}^{\prime}=f_{\text {crys }}^{*}(\mathcal{E})$ be the inverse image of $\mathcal{E}$, which is the crystal on $Y^{\prime}$ corresponding to the inverse image connection $\left(\mathcal{E}_{n}^{\prime}, \nabla_{n}^{\prime}\right)=\left(\tilde{u}_{n}^{*}\left(\mathcal{E}_{n}\right), \tilde{u}_{n}^{*}\left(\nabla_{n}\right)\right)$ on $P_{n}^{\prime}, \tilde{u}_{n}$ denoting the morphism of ringed spaces $\left(P_{n}^{\prime}, \mathcal{P}_{n}^{\prime}\right) \rightarrow\left(P_{n}, \mathcal{P}_{n}\right)$ defined by $u$. Then the following square is commutative:

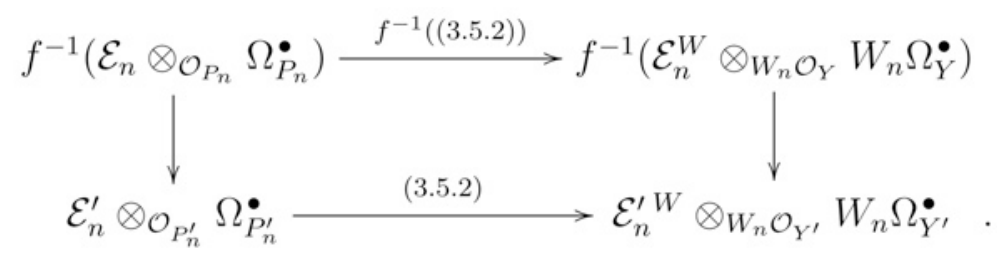

When $\mathcal{E}$ is a crystal on $Y / W$, there is a similar diagram based on (3.5.3).

When $f$ is the identity on $Y$, the right vertical arrow of diagram (3.5.4) is the identity, and the left vertical arrow is a quasi-isomorphism which corresponds in the derived category to the identity on crystalline cohomology, via the canonical identifications (3.4.1) between the crystalline cohomology of $\mathcal{E}$ and the de Rham cohomologies of $\mathcal{E}_{n}$ and $\mathcal{E}_{n}^{\prime}$ on $P_{n}$ and $P_{n}^{\prime}$. It follows that, in $D^{\mathrm{b}}\left(Y, W_{n}\right)$ and $D^{\mathrm{b}}(Y, W)$, the morphisms (3.5.2) and (3.5.3) do not depend on the choice of $(\mathbb{P}, F)$ when they are viewed as morphisms from crystalline cohomology to de Rham-Witt cohomology via (3.4.1) and (3.4.3).

Finally, using Čech complexes (or cohomological descent) as in the case of cohomology with constant coefficients, these functorialities also allow to extend to the general case (i.e., without assuming the existence of an embedding $Y \hookrightarrow(\mathbb{P}, F)$ as above) the construction of the morphisms (3.5.2) and (3.5.3) as morphisms

$$
\begin{gathered}
\mathbb{R} u_{Y / W_{n} *} \mathcal{E} \longrightarrow \mathcal{E}_{n}^{W} \otimes_{W_{n} \mathcal{O}_{Y}} W_{n} \Omega_{Y}^{\bullet}, \\
\mathbb{R} u_{Y / W *} \mathcal{E} \longrightarrow \widehat{\mathcal{E}}^{W} \widehat{\otimes}_{W \mathcal{O}_{Y}} W \Omega_{Y}^{\bullet}
\end{gathered}
$$

in the corresponding derived categories. Taking global sections, one ob- 
tains morphisms

$$
\begin{gathered}
\mathbb{R} \Gamma_{\text {crys }}\left(Y / W_{n}, \mathcal{E}\right) \longrightarrow \mathbb{R} \Gamma\left(Y, \mathcal{E}_{n}^{W} \otimes_{W_{n} \mathcal{O}_{Y}} W_{n} \Omega_{Y}^{\bullet}\right), \\
\mathbb{R} \Gamma_{\text {crys }}(Y / W, \mathcal{E}) \longrightarrow \mathbb{R} \Gamma\left(Y, \widehat{\mathcal{E}}^{W} \widehat{\otimes}_{W \mathcal{O}_{Y}} W \Omega_{Y}^{\bullet}\right)
\end{gathered}
$$

(as usual, we assume in (3.5.6) and (3.5.8) that $\mathcal{E}$ is quasi-coherent).

Our basic comparison theorem between crystalline and de Rham-Witt cohomologies with coefficients is then the following:

THEOREM 3.6. Let $k$ be a perfect field of characteristic $p$, and let $Y$ be a smooth $k$-scheme. For some $n \in \mathbb{N}$, let $\mathcal{E}$ be a crystal on $Y / W_{n}$. If $\mathcal{E}$ is flat over $W_{n}$, the comparison morphisms (3.5.5) and (3.5.7) are isomorphisms respectively in $D^{\mathrm{b}}\left(Y, W_{n}\right)$ and $D^{\mathrm{b}}\left(W_{n}\right)$.

This generalizes earlier results of Étesse [11, Théorème 2.1] and Langer-Zink [18, Theorem 3.8], where $\mathcal{E}$ is assumed to be flat over $\mathcal{O}_{Y / W_{n}}$.

The first assertion of the theorem implies the second one. To prove the first one, one can make further reductions. Indeed, taking an affine open covering $\left(U_{\alpha}\right)$ of $Y$, choosing closed immersions of each $U_{\alpha}$ in a smooth formal scheme $\mathbb{P}_{\alpha}$ endowed with a Frobenius lifting, and using the definition of (3.5.5) as a morphism of $D^{\mathrm{b}}\left(Y, W_{n}\right)$ thanks to Čech resolutions (or to cohomological descent), it suffices to prove that, on each intersection $U_{\underline{\alpha}}$, the corresponding morphism (3.5.2) of the category of complexes is a quasi-isomorphism. So we are reduced to proving that, when $Y$ can be embedded in a smooth formal scheme $\mathbb{P}$ endowed with a Frobenius lifting, the morphism of complexes (3.5.2) is a quasi-isomorphism. This is a local statement on $Y$. As this morphism does not depend in the derived category on the choice of $(\mathbb{P}, F)$, we may localize and assume that $Y$, together with its Frobenius endomorphism, can be lifted as a smooth formal scheme $Y$ over $W$, with reduction $Y_{n}$ over $W_{n}$. Thus we may assume that $\mathbb{P}=\mathbb{Y}$, and it suffices to prove the following local form of the theorem:

THEOREM 3.7. Let $k$ be a perfect field of characteristic $p$, and let $Y$ be a smooth $k$-scheme. Assume that there exists a smooth formal scheme $Y$ over $W$ lifting $Y$, with reduction $Y_{n}$ over $W_{n}$, and a semi-linear endomorphism $F$ of $Y$ lifting the absolute Frobenius endomorphism of $Y$. For some $n \in \mathbb{N}$, let $\mathcal{E}$ be a crystal on $Y / W_{n}$. If $\mathcal{E}$ is flat over $W_{n}$, the morphism of complexes

$$
\mathcal{E}_{n} \otimes_{\mathcal{O}_{Y_{n}}} \Omega_{Y_{n}}^{\bullet} \rightarrow \mathcal{E}_{n}^{W} \otimes_{W_{n} \mathcal{O}_{Y}} W_{n} \Omega_{Y}^{\bullet}
$$

defined by (3.5.2), is a quasi-isomorphism. 
PRoof. If we endow the complex $\mathcal{E} \otimes \Omega_{Y_{n}}^{\bullet}$ with the $p$-adic filtration and the complex $\mathcal{E}_{n}^{W} \otimes_{W_{n} \mathcal{O}_{Y}} W_{n} \Omega_{Y}^{\bullet}$ with the canonical filtration defined in (2.6.3), the morphism (3.5.2) becomes a morphism of filtered complexes, and we are reduced to proving that the morphisms

$$
p^{i} \mathcal{E} \otimes_{\mathcal{O}_{Y_{n}}} \Omega_{Y_{n}}^{\bullet} / p^{i+1} \mathcal{E} \otimes_{\mathcal{O}_{Y_{n}}} \Omega_{Y_{n}}^{\bullet} \rightarrow \operatorname{gr}^{i}\left(\mathcal{E}_{n}^{W} \otimes_{W_{n} \mathcal{O}_{Y}} W_{n} \Omega_{Y}^{\bullet}\right)
$$

are quasi-isomorphisms for all $i$.

As the morphism (3.5.2) is $h_{F^{-}}$-semi-linear, the morphism (3.7.1) is $t_{F^{-}}$ semi-linear. If we denote as before by $\bar{t}_{F}$ the reduction modulo $p$ of $t_{F}$, we can view $\operatorname{gr}^{i} W_{n} \Omega_{Y}^{\bullet}$ and $\operatorname{gr}^{i}\left(\mathcal{E}_{n}^{W} \otimes_{W_{n} \mathcal{O}_{Y}} W_{n} \Omega_{Y}^{\bullet}\right)$ as $\mathcal{O}_{Y}$-modules through $\bar{t}_{F}$. The morphism (3.7.2) is then $\mathcal{O}_{Y}$-linear.

Using (2.7.1), (2.2.2), and the fact that $\mathrm{gr}^{i} W_{n} \Omega_{Y}^{\bullet}$ is annihilated by $p$, we obtain natural linear isomorphisms of complexes

$$
\mathcal{E}_{1} \otimes_{\mathcal{O}_{Y}} \operatorname{gr}^{i} W_{n} \Omega_{Y}^{\bullet} \stackrel{\sim}{\longrightarrow} \operatorname{gr}^{i}\left(\mathcal{E}_{n}^{W} \otimes_{W_{n} \mathcal{O}_{Y}} W_{n} \Omega_{Y}^{\bullet}\right) .
$$

Multiplication by $p^{i}$ induces a linear morphism of complexes

$$
\underline{p}^{i}: \mathcal{E}_{1} \otimes_{\mathcal{O}_{Y}} \operatorname{gr}^{0} W_{n} \Omega_{Y}^{\bullet} \rightarrow \mathcal{E}_{1} \otimes_{\mathcal{O}_{Y}} \operatorname{gr}^{i} W_{n} \Omega_{Y}^{\bullet} .
$$

On the other hand, since $\mathcal{E}_{n}$ is flat over $W_{n}$, multiplication by $p^{i}$ induces a linear isomorphism of complexes

$$
\underline{p}^{i}: \mathcal{E}_{1} \otimes_{\mathcal{O}_{Y}} \Omega_{Y}^{\bullet} \stackrel{\sim}{\longrightarrow} p^{i} \mathcal{E} \otimes_{\mathcal{O}_{Y_{n}}} \Omega_{Y_{n}}^{\bullet} / p^{i+1} \mathcal{E} \otimes_{\mathcal{O}_{Y_{n}}} \Omega_{Y_{n}}^{\bullet} .
$$

As in [14, II 1.4], these morphisms fit in commutative diagrams

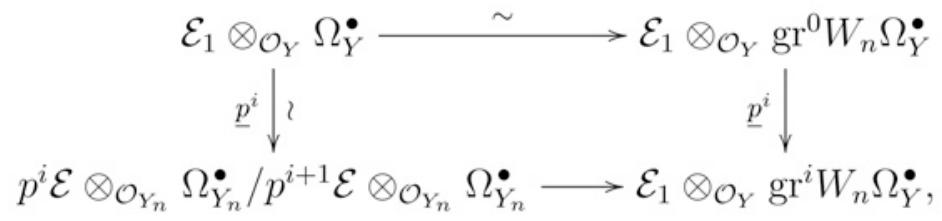

in which the upper horizontal isomorphism follows from the identification between $\operatorname{gr}^{0} W_{n} \Omega_{Y}^{\circ}$ and $\Omega_{Y}^{\circ}$. Hence it suffices to prove that the morphisms

$$
\underline{p}^{i}: \mathcal{E}_{1} \otimes_{\mathcal{O}_{Y}} \Omega_{Y}^{\bullet} \rightarrow \mathcal{E}_{1} \otimes_{\mathcal{O}_{Y}} \operatorname{gr}^{i} W_{n} \Omega_{Y}^{\bullet}
$$

are quasi-isomorphisms for all $i$.

As in the proof of Lemma 2.5, we can endow $\mathcal{E}_{1}$ with an exhaustive filtration by horizontal submodules $\mathcal{E}_{1}^{m}$ such that $\mathcal{E}_{1}^{m} / \mathcal{E}_{1}^{m-1}$ has $p$-curvature 0 . Since the filtration is exhaustive, it suffices to prove that the above 
morphism is a quasi-isomorphism when $\mathcal{E}_{1}$ is replaced by $\mathcal{E}_{1}^{m}$ for any $m$, and it is even sufficient to prove it for the quotients $\mathcal{E}_{1}^{m} / \mathcal{E}_{1}^{m-1}$. As their connections have $p$-curvature 0 , they are of the form $F^{*} \mathcal{F}^{m}$ for some $\mathcal{O}_{Y^{-}}$ modules $\mathcal{F}^{m}$, as $\mathcal{O}_{Y}$-modules with connection. So it is enough to check that the morphisms of complexes

$$
\underline{p}^{i}: \mathcal{F}^{m} \otimes_{\mathcal{O}_{Y}} F_{*} \Omega_{Y}^{\bullet} \rightarrow \mathcal{F}^{m} \otimes_{\mathcal{O}_{Y}} F_{*} \mathrm{gr}^{i} W_{n} \Omega_{Y}^{\bullet}
$$

are quasi-isomorphisms. From [14, I 3.14], we know that the morphisms $p^{i}: \Omega_{Y}^{\bullet} \rightarrow \operatorname{gr}^{i} W_{n} \Omega_{Y}^{\bullet}$ are quasi-isomorphisms, and both complexes are linear complexes of locally free finitely generated $\mathcal{O}_{Y}$-modules when $\mathcal{O}_{Y}$ acts by $F$ on $\Omega_{Y}^{\bullet}$ and by $\bar{F}: \mathcal{O}_{Y} \rightarrow W_{n} \mathcal{O}_{Y} / p W_{n} \mathcal{O}_{Y}$ on $\operatorname{gr}^{i} W_{n} \Omega_{Y}^{\bullet}$ [14, I, 3.9]. By Lemma $2.4, \bar{F}=\bar{t}_{F} \circ F$, so the $\mathcal{O}_{Y}$-action on $F_{*} g^{i} W_{n} \Omega_{Y}^{\bullet}$ in (3.7.3) is the one given by $\bar{F}$. Moreover, the connection on $F^{*} \mathcal{F}^{m}$ is simply $\operatorname{Id}_{\mathcal{F}^{m}} \otimes d$, where the tensor product is taken through $F$. Therefore these quasi-isomorphisms remain quasi-isomorphisms after tensorizing with $\mathcal{F}^{m}$.

COROLLARY 3.8. Let $k$ be a perfect field of characteristic $p$, and let $Y$ be a smooth $k$-scheme. Let $\mathcal{E}$ be a crystal on $Y / W$, flat over $W$ and such that the $\mathcal{O}_{Y}$-module $\mathcal{E}_{1}$ defined by $\mathcal{E}$ is quasi-coherent.

(i) If there exists a smooth formal scheme $Y$ over $W$ lifting $Y$ and $a$ semi-linear endomorphism $F: Y \rightarrow Y$ lifting the absolute Frobenius endomorphism of $Y$, then the morphism of complexes

$$
\widehat{\mathcal{E}} \otimes_{\mathcal{O}_{Y}} \Omega_{Y}^{\bullet} \rightarrow \widehat{\mathcal{E}}^{W} \widehat{\otimes}_{W \mathcal{O}_{Y}} W \Omega_{Y}^{\bullet},
$$

defined by (3.5.3), is a quasi-isomorphism.

(ii) In the general case, the comparison morphisms (3.5.6) and (3.5.8) are isomorphisms in $D^{\mathrm{b}}(Y, W)$ and $D^{\mathrm{b}}(W)$.

Proof. The quasi-coherence of $\mathcal{E}_{1}$ and the flatness of $\widehat{\mathcal{E}}$ over $W$ imply that $\mathcal{E}_{n} \simeq \widehat{\mathcal{E}} / p^{n} \widehat{\mathcal{E}}$ is quasi-coherent for all $n$. Then the terms of the two complexes of inverse systems $\mathcal{E}_{\bullet} \otimes \Omega_{Y}^{\bullet}$ and $\mathcal{E}_{\bullet}^{W} \otimes_{W_{\bullet} \mathcal{O}_{Y}} W_{\bullet} \Omega_{Y}^{\bullet}$ have vanishing cohomology on open affine subsets and surjective transition maps. Therefore, these are complexes with lim-acyclic terms, and we obtain equalities

$$
\begin{aligned}
& \mathbb{R} \lim _{\leftarrow}\left(\mathcal{E}_{\bullet} \otimes \Omega_{Y_{\bullet}}^{\bullet}\right)=\lim _{\leftarrow}\left(\mathcal{E}_{\bullet} \otimes \Omega_{Y_{\bullet}}^{\bullet}\right), \\
& \mathbb{R} \lim _{\leftarrow}\left(\mathcal{E}_{\bullet}^{W} \otimes_{W \cdot \mathcal{O}_{Y}} W_{\bullet} \Omega_{Y}^{\bullet}\right)=\lim _{\leftarrow}\left(\mathcal{E}_{\bullet}^{W} \otimes_{W \cdot \mathcal{O}_{Y}} W \cdot \Omega_{Y}^{\bullet}\right) .
\end{aligned}
$$


When $n$ varies, the quasi-isomorphisms (3.7.1) provide a quasi-isomorphism of complexes of inverse systems. Applying the $\mathbb{R} \lim$ functor, it follows that (3.8.1) is a quasi-isomorphism.

As in the proof of Theorem 3.6, assertion (ii) follows from assertion (i) by taking an affine covering of $Y$, choosing closed immersions of the open subsets of the covering into smooth formal schemes endowed with Frobenius liftings, and representing the morphism (3.5.3) in $D^{\mathrm{b}}(Y, W)$ thanks to the associated Čech resolutions.

3.9. As an example of application of Theorem 3.6, we now show that, for local complete intersections, crystalline cohomology can be computed using the de Rham-Witt complexes of smooth embeddings. We first recall a few facts about crystalline direct images by a closed immersion [7, Prop. 6.2].

Let $Y$ be a $k$-scheme, and let $i: X \hookrightarrow Y$ be a closed immersion defined by an ideal $\mathcal{I} \subset \mathcal{O}_{Y}$. For any PD-thickening $(U, T, \delta)$ of an open subset $U \subset Y$, we denote by $\mathcal{I}_{T}$ the ideal of $X \cap U$ in $T$. Let $i_{\text {crys } *}$ be the direct image functor from the category of sheaves on $\operatorname{Crys}\left(X / W_{n}\right)$ (resp. $\operatorname{Crys}(X / W))$ to the category of sheaves on $\operatorname{Crys}\left(Y / W_{n}\right)(\operatorname{resp}$. $\operatorname{Crys}(Y / W))$. Then:

a) The sheaf $i_{\text {crys } *} \mathcal{O}_{X / W_{n}}$ (resp. $\left.i_{\text {crys } *} \mathcal{O}_{X / W}\right)$ is a crystal in $\mathcal{O}_{Y / W_{n}}$-algebras (resp. $\mathcal{O}_{Y / W}$-algebras) supported in $X$. Its evaluation on a PDthickening $(U, T, \delta)$ is given by

$$
\left(i_{\text {crys } *} \mathcal{O}_{X / W_{n}}\right)_{T}=\mathcal{P}_{\delta}\left(\mathcal{I}_{T}\right)
$$

(resp. $\left.\left(i_{\text {crys } *} \mathcal{O}_{X / W}\right)_{T}\right)$, where $\mathcal{P}_{\delta}$ denotes the divided power envelope with compatibility with $\delta$.

b) For all $q \geq 1, R^{q} i_{\text {crys } *} \mathcal{O}_{X / W_{n}}=0\left(\operatorname{resp} . R^{q} i_{\text {crys } *} \mathcal{O}_{X / W}=0\right)$.

Theorem 3.10. Let $Y$ be a smooth $k$-scheme, and let $i: X \hookrightarrow Y$ be a regular immersion defined by an ideal $\mathcal{I} \subset \mathcal{O}_{Y}$. For each $n \geq 1$, set $W_{n} \mathcal{I}=\operatorname{Ker}\left(W_{n} \mathcal{O}_{Y} \rightarrow W_{n} \mathcal{O}_{X}\right)$. Let $\mathcal{P}_{n}^{W}$ be the PD-envelope of $W_{n} \mathcal{I}$ with compatibility with the canonical divided powers of $V W_{n-1} \mathcal{O}_{Y}$, and let $\widehat{\mathcal{P}}^{W}=\lim _{\overleftarrow{n}} \mathcal{P}_{n}^{W}$. There exists functorial isomorphisms

$$
\begin{aligned}
\mathbb{R} u_{X / W_{n} *} \mathcal{O}_{X / W_{n}} \stackrel{\sim}{\longrightarrow} \mathcal{P}_{n}^{W} \otimes_{W_{n} \mathcal{O}_{Y}} W_{n} \Omega_{Y}^{\bullet}, \\
\mathbb{R} u_{X / W *} \mathcal{O}_{X / W} \stackrel{\sim}{\longrightarrow} \widehat{\mathcal{P}}^{W} \widehat{\otimes}_{W \mathcal{O}_{Y}} W \Omega_{Y}^{\bullet},
\end{aligned}
$$


respectively in $D^{\mathrm{b}}\left(X, W_{n}\right)$ and $D^{\mathrm{b}}(X, W)$, and

$$
\begin{gathered}
\mathbb{R} \Gamma_{\text {crys }}\left(X / W_{n}, \mathcal{O}_{X / W_{n}}\right) \stackrel{\sim}{\longrightarrow} \Gamma\left(X, \mathcal{P}_{n}^{W} \otimes_{W_{n} \mathcal{O}_{Y}} W_{n} \Omega_{Y}^{\bullet}\right), \\
\mathbb{R} \Gamma_{\text {crys }}\left(X / W, \mathcal{O}_{X / W}\right) \stackrel{\sim}{\longrightarrow} \Gamma\left(X, \widehat{\mathcal{P}}^{W} \widehat{\otimes}_{W \mathcal{O}_{Y}} W \Omega_{Y}^{\bullet}\right),
\end{gathered}
$$

respectively in $D^{\mathrm{b}}\left(W_{n}\right)$ and $D^{\mathrm{b}}(W)$.

Proof. As $u_{X / W_{n}}=u_{Y / W_{n}} \circ i_{\text {crys }}$, we get from $\left.3.9 \mathrm{~b}\right)$ a canonical isomorphism

$$
\mathbb{R} u_{X / W_{n} *} \mathcal{O}_{X / W_{n}} \stackrel{\sim}{\longrightarrow} \mathbb{R} u_{Y / W_{n} *}\left(i_{\text {crys } *} \mathcal{O}_{X / W_{n}}\right) .
$$

To define (3.10.1), it suffices to check that the crystal $i_{\text {crys } *} \mathcal{O}_{X / W_{n}}$ is flat over $W_{n}$ and that its evaluation on $W_{n} Y$ is canonically isomorphic to $\mathcal{P}_{n}^{W}$ : Theorem 3.6 will then provide an isomorphism

$$
\mathbb{R} u_{Y / W_{n} *}\left(i_{\text {crys } *} \mathcal{O}_{X / W_{n}}\right) \stackrel{\sim}{\longrightarrow} \mathcal{P}_{n}^{W} \otimes_{W_{n} \mathcal{O}_{Y}} W_{n} \Omega_{Y}^{\bullet}
$$

and we will obtain (3.10.1) as the composition of (3.10.5) and (3.10.6).

To check the first claim, consider an affine open subset $U \subset Y$ on which $\mathcal{I}$ is defined by a regular sequence $\left(t_{1}, \ldots, t_{d}\right)$. Let $U_{n}$ be a smooth lifting of $U$ over $W_{n}$ and $\left.\tilde{t}_{1}, \ldots, \tilde{t}_{d}\right)$ a sequence of sections of $\mathcal{O}_{U_{n}}$ lifting $\left(t_{1}, \ldots, t_{d}\right)$. It is easy to check that $\left(\tilde{t}_{1}, \ldots, \tilde{t}_{d}\right)$ is a regular sequence in $\mathcal{O}_{U_{n}}$ and that $\mathcal{O}_{U_{n}} /\left(\tilde{t}_{1}, \ldots, \tilde{t}_{d}\right)$ is flat over $W_{n}$. The ideal of $X$ in $U_{n}$ is $\mathcal{I}_{n}=\left(p, \tilde{t}_{1}, \ldots, \tilde{t}_{d}\right)$, and, by (3.9.1), the evaluation of $i_{\text {crys } *} \mathcal{O}_{X / W}$ on $U_{n}$ is the divided power envelope $\mathcal{P}_{n}$ of $\mathcal{I}_{n}$ with compatibility with the divided powers of $(p)$. This divided power envelope is also the divided power envelope of the ideal $\left(\tilde{t}_{1}, \ldots, \tilde{t}_{d}\right)$ with compatibility with the divided powers of $(p)$, and, by $[6$, 2.3.3 and 2.3.4], it is flat over $W_{n}$. Therefore, the crystal $i_{\text {crys } *} \mathcal{O}_{X / W}$ is flat over $W_{n}$.

On the other hand, (3.9.1) shows that the evaluation of $i_{\text {crys } *} \mathcal{O}_{X / W_{n}}$ on $W_{n} Y$ is the PD-envelope $\mathcal{P}_{\text {can }}\left(\mathcal{I}_{W_{n} Y}\right)$, where the subscript can denotes compatibility with the canonical divided powers of $V W_{n-1} \mathcal{O}_{Y}$. As the ideal $\mathcal{I}_{W_{n} Y}$ can be written

$$
\mathcal{I}_{W_{n} Y}=W_{n} \mathcal{I}+V W_{n-1} \mathcal{O}_{Y}
$$

the second claim follows.

To define the isomorphism (3.10.2), one constructs the family of isomorphisms (3.10.1) for variable $n$ so as to obtain an isomorphism in the derived category of inverse systems. The quasi-coherence of PD-envelopes implies that, for all $n, \mathcal{P}_{n}^{W} \otimes_{W_{n} \mathcal{O}_{Y}} W_{n} \Omega_{Y}^{\bullet}$ is a quasi-coherent $W_{n} \mathcal{O}_{Y^{-}}$ 
module. Therefore, the inverse system with surjective transitions maps $\left(\mathcal{P}_{n}^{W} \otimes_{W_{n} \mathcal{O}_{Y}} W_{n} \Omega_{Y}^{\bullet}\right)_{n}$ is lim-acyclic, and applying the $\underset{\leftarrow}{\mathbb{R}} \lim _{\leftarrow}$ functor provides (3.10.2). Taking global sections, one gets (3.10.3) and (3.10.4).

\section{Rigid cohomology as a limit of crystalline cohomologies}

Our goal now is to apply the comparison theorems of the previous section to the computation of rigid cohomology with compact supports in terms of de Rham-Witt complexes, for a separated $k$-scheme of finite type. The key case is the case of a proper $k$-scheme, and our method is based on the interpretation of the rigid cohomology of such a scheme as a limit of suitable crystalline cohomologies (see [10, section 2] for a closely related point of view).

4.1. We briefly recall some basic constructions used in the definition of rigid cohomology for proper $k$-schemes (see [2], [4], [19] for details and proofs).

Let $\mathbb{P}$ be a smooth formal $W$-scheme, and let $\mathbb{P}_{K}$ denote its generic fibre in Raynaud's sense, which is a rigid analytic space over $K$. If $P$ is affine, defined by $\mathbb{P}=\operatorname{Spf} A$ where $A$ is topologically of finite type over $W$, then $\mathbb{P}_{K}$ is the affinoid space $A_{K}$ defined by the Tate algebra $A_{K}$. In general, $\mathbb{P}_{K}$ is endowed with a specialisation morphism sp $: \mathbb{P}_{K} \rightarrow \mathbb{P}$, which is a continuous map such that $\mathrm{sp}^{-1}(\mathbb{U})=U_{K}$ for any open subset $U \subset P$. By construction, we have

$$
\operatorname{sp}_{*} \mathcal{O}_{\mathbb{P}_{K}}=\mathcal{O}_{\mathrm{P}, K}:=\mathcal{O}_{\mathrm{P}} \otimes K .
$$

Let $P$ be the special fibre of $\mathbb{P}$, and let $X \hookrightarrow P$ be a closed subscheme defined by an ideal $\mathcal{J} \subset \mathcal{O}_{\mathrm{P}}$. The tube of $X$ in $\mathbb{P}_{K}$ is the subset ]$X\left[\mathrm{P}:=\mathrm{sp}^{-1}(X)\right.$. If $\mathrm{U}=\operatorname{Spf} A \subset \mathbb{P}$ is an affine open subset, and if $f_{1}, \ldots, f_{r} \in A$ is a family of generators of $\Gamma(\mathbb{U}, \mathcal{J})$, then

$$
] X L_{\mathbb{P}} \cap \mathbb{U}_{K}=\left\{x \in \mathbb{U}_{K}|\forall i,| f_{i}(x) \mid<1\right\},
$$

where, for a point $x \in \mathbb{U}_{K}$ corresponding to a maximal ideal $\mathfrak{m} \subset A_{K},\left|f_{i}(x)\right|$ is the absolute value of the class of $f_{i}$ in the residue field $K(x)=A_{K} / \mathfrak{m}$. In particular, $] X\left[\mathrm{P}\right.$ is an admissible open subset of $\mathbb{P}_{K}$.

As the specialization morphism sp : $\mathbb{P}_{K} \rightarrow \mathbb{P}$ maps $] X$ [p to $X$, one defines a sheaf of $\mathcal{O}_{\mathbb{P}, K}$-algebras supported on $X$ by setting

$$
\mathcal{A}_{X, \mathrm{P}}=\operatorname{sp}_{*} \mathcal{O}_{] X[\mathrm{P}} .
$$


For any affine open subset $U \subset \mathbb{P}$ and any $j$, there is a canonical isomorphism $\Gamma\left(\cup_{K}, \Omega_{\mathrm{P}_{K}}^{j}\right) \simeq \Gamma\left(\mathbb{U}, \Omega_{\mathrm{P}}^{j}\right) \otimes K$, which is compatible with differentiation. It follows that the canonical derivation of $\mathcal{O}_{] X[}$ endows $\mathcal{A}_{X, \mathrm{P}}$ with an integrable connection. Therefore, one can define the de Rham complex $\mathcal{A}_{X, \mathrm{P}} \otimes \Omega_{\mathrm{P}}^{\bullet}$, and, taking into account that each $\Omega_{\mathrm{P}}^{j}$ is locally free of finite rank, one gets a canonical isomorphism of complexes

$$
\mathcal{A}_{X, \mathrm{P}} \otimes_{\mathcal{O}_{\mathrm{P}}} \Omega_{\mathrm{P}}^{\bullet} \simeq \operatorname{sp}_{*}\left(\Omega_{] X{ }_{[\mathrm{P}}}^{\bullet}\right) .
$$

If $U \subset \mathbb{P}$ is affine, it follows from (4.1.1) that $] X\left[p \cap \mathbb{U}_{K}\right.$ is quasi-Stein [17, Definition 2.3], hence Kiehl's vanishing theorem implies that $R^{i} \operatorname{sp}_{*}\left(\Omega_{] X[\mathrm{P}}^{j}\right)=0$ for all $j$ and all $i \geq 1$. Therefore, we obtain

$$
\mathcal{A}_{X, \mathrm{P}} \otimes_{\mathcal{O}_{\mathrm{P}}} \Omega_{\mathrm{P}}^{\bullet} \simeq \operatorname{Rsp}_{*}\left(\Omega_{] X[\mathrm{P}}^{\bullet}\right)
$$

in the derived category $D^{\mathrm{b}}(X, K)$.

As an object of $D^{\mathrm{b}}(X, K)$, the complex $\mathcal{A}_{X, \mathrm{P}} \otimes \Omega_{\mathrm{P}}^{\bullet}$ does not depend (up to canonical isomorphism) on the choice of the embedding $X \hookrightarrow \mathbb{P}$, and it is functorial with respect to $X[4,1.5]$. We will use the notation

$$
\mathbb{R} \underline{\Gamma}_{\text {rig }}(X / K):=\mathcal{A}_{X, \mathrm{P}} \otimes \Omega_{\mathrm{P}}^{\bullet}
$$

to denote $\mathcal{A}_{X, \mathrm{P}} \otimes \Omega_{\mathrm{P}}^{\bullet}$ as an object of $D^{\mathrm{b}}(X, K)$. Assuming that $X$ is a proper $k$-scheme and can be embedded in a smooth formal scheme $\mathrm{P}$ as above, its rigid cohomology is defined by setting

$$
\begin{aligned}
\mathbb{R} \Gamma_{\text {rig }}(X / K) & =\mathbb{R} \Gamma\left(X, \mathbb{R} \underline{\Gamma}_{\text {rig }}(X / K)\right) \\
& =\mathbb{R} \Gamma\left(X, \mathcal{A}_{X, \mathrm{P}} \otimes \Omega_{\mathrm{P}}^{\bullet}\right) \simeq \mathbb{R} \Gamma(] X\left[_{\mathrm{p}}, \Omega_{] X[\mathrm{P}}^{\bullet}\right) .
\end{aligned}
$$

In the general case, where such an embedding might not exist, one can generalize this definition using a Čech style construction analogous to the description of the complex $\mathbb{R} u_{X / W *} \mathcal{O}_{X / W}$ given in 3.1 (see also [13, pp. 2829], where the analogous construction is used for algebraic de Rham cohomology in characteristic 0 ). One can choose a covering of $X$ by affine open subsets $U_{\alpha}$, and, for each $\alpha$, a closed immersion of $U_{\alpha}$ in a smooth affine formal scheme $\mathbb{P}_{\alpha}$ over $W$. Then the diagonal immersions for the finite intersections

$$
U_{\underline{\alpha}}:=U_{\alpha_{0}} \cap \ldots \cap U_{\alpha_{i}} \hookrightarrow \mathbb{P}_{\underline{\alpha}}:=\mathbb{P}_{\alpha_{0}} \times_{W} \cdots \times_{W} \mathbb{P}_{\alpha_{i}}
$$

provide algebras $\mathcal{A}_{U_{\underline{\alpha}}, \mathbb{P}_{\underline{\alpha}}}$. From the de Rham complexes of the products $\mathbb{P}_{\underline{\alpha}}$ with coefficients in these algebras, one can build as in 3.1 a Cech double complex. As an object of $D(X, K)$, the associated total complex does not depend on the choices, and it is functorial with respect to $X$. It will still be 
denoted by $\mathbb{R} \underline{\Gamma}_{\text {rig }}(X / K)$. When $X$ is proper, its rigid cohomology is again defined by $\mathbb{R} \Gamma_{\text {rig }}(X / K):=\mathbb{R} \Gamma\left(X, \mathbb{R} \underline{\Gamma}_{\text {rig }}(X / K)\right)$.

4.2. To construct a de Rham-Witt complex computing rigid cohomology for proper varieties, we will use a description of the algebra $\mathcal{A}_{X, \mathrm{p}}$ based on PD-envelopes.

Let $\mathcal{J} \subset \mathcal{O}_{\mathrm{P}}$ be a coherent ideal. We denote by $\mathcal{P}(\mathcal{J})$ the divided power envelope of $\mathcal{J}$ (with compatibility with the natural divided powers of $p$ ). By definition, $\mathcal{P}(\mathcal{J})$ is the sheaf of $\mathcal{O}_{\mathrm{p}}$-algebras associated to the presheaf $\mathbb{U} \mapsto \mathcal{P}(\Gamma(\mathbb{U}, \mathcal{J}))$, where $\mathcal{P}(\Gamma(\mathbb{U}, \mathcal{J}))$ is the PD-envelope of the ideal $\Gamma(\cup, \mathcal{J}) \subset \Gamma\left(\cup, \mathcal{O}_{\mathrm{P}}\right)$. However, the formation of PD-envelopes commutes with flat ring extensions [7, 3.21]. Since $\mathcal{J}$ is coherent over $\mathcal{O}_{\mathrm{P}}$ and $\Gamma\left(\mathbb{V}, \mathcal{O}_{\mathrm{P}}\right)$ is flat over $\Gamma\left(\mathbb{U}, \mathcal{O}_{\mathrm{P}}\right)$ for any couple of affine open subsets $\mathrm{V} \subset \mathbb{U}$ in $\mathbb{P}$, it follows that the functor $\mathbb{U} \mapsto \mathcal{P}(\Gamma(\mathbb{U}, \mathcal{J}))$ satisfies the glueing condition on the category of affine open subsets of $P$. This implies that

$$
\Gamma(\bigcup, \mathcal{P}(\mathcal{J}))=\mathcal{P}(\Gamma(\bigcup, \mathcal{J}))
$$

for any affine open subset $U \subset \mathbb{P}$.

We denote by $\widehat{\mathcal{P}}(\mathcal{J})$ the $p$-adic completion of $\mathcal{P}(\mathcal{J})$. As divided power envelopes may have some $p$-torsion, we also introduce the ideal $\mathcal{T}(\mathcal{J})$ of $p$ torsion sections of $\mathcal{P}(\mathcal{J})$, the quotient $\overline{\mathcal{P}}(\mathcal{J})=\mathcal{P}(\mathcal{J}) / \mathcal{T}(\mathcal{J})$, and its $p$-adic completion $\widetilde{\mathcal{P}}(\mathcal{J})$. Note that the natural connection of the divided power envelope $\mathcal{P}(\mathcal{J})$ extends to the algebras $\widehat{\mathcal{P}}(\mathcal{J}), \overline{\mathcal{P}}(\mathcal{J})$ and $\widetilde{\mathcal{P}}(\mathcal{J})$.

We first observe that these algebras have quasi-coherent reduction $\bmod p^{n}$ :

Lemma 4.3. Let $\mathcal{J} \subset \mathcal{O}_{\mathbb{P}}$ be a coherent ideal, $n \geq 1$ an integer, and $P_{n}$ the reduction of $\mathrm{P}$ on $W_{n}$. Define

$$
\begin{aligned}
\mathcal{P}_{n}(\mathcal{J})= & \mathcal{P}(\mathcal{J}) / p^{n} \mathcal{P}(\mathcal{J}), \quad \overline{\mathcal{P}}_{n}(\mathcal{J})=\overline{\mathcal{P}}(\mathcal{J}) / p^{n} \overline{\mathcal{P}}(\mathcal{J}), \\
& \mathcal{T}_{n}(\mathcal{J})=\operatorname{Ker}\left(p^{n}: \mathcal{P}(\mathcal{J}) \rightarrow \mathcal{P}(\mathcal{J})\right) .
\end{aligned}
$$

Then:

(i) $\mathcal{P}_{n}(\mathcal{J})$ and $\overline{\mathcal{P}}_{n}(\mathcal{J})$ are quasi-coherent $\mathcal{O}_{P_{n}}$-algebras.

(ii) $\mathcal{T}_{n}(\mathcal{J})$ is a quasi-coherent $\mathcal{O}_{P_{n}}$-module.

Proof. As the divided powers of $\mathcal{P}(\mathcal{J})$ are compatible with the divided powers of $p$, there is a canonical isomorphism

$$
\mathcal{P}_{n}(\mathcal{J})=\mathcal{P}(\mathcal{J}) / p^{n} \mathcal{P}(\mathcal{J}) \stackrel{\sim}{\longrightarrow} \mathcal{P}\left(\mathcal{J}_{n}\right)
$$


where $\left.\mathcal{J}_{n}=\mathcal{J O}_{P_{n}}[7,3.20-8)\right]$. The ideal $\mathcal{J}_{n}$ is quasi-coherent over $\mathcal{O}_{P_{n}}$, and therefore the $\mathcal{O}_{P_{n}}$-algebra $\mathcal{P}\left(\mathcal{J}_{n}\right)$ is also quasi-coherent [7, 3.30], which proves the first claim.

Since $\mathcal{T}(\mathcal{J})=\cup_{i} \mathcal{T}_{i}(\mathcal{J})$, the commutative diagrams

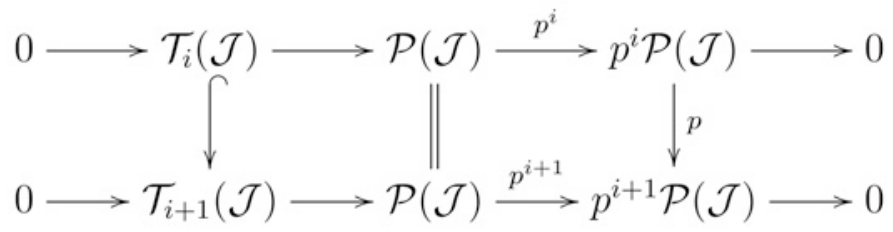

provide an isomorphism

$$
\lim _{\vec{i}} p^{i} \mathcal{P}(\mathcal{J}) \stackrel{\sim}{\longrightarrow} \overline{\mathcal{P}}(\mathcal{J}),
$$

in which the transition maps of the direct system are defined by multiplication by $p$. Therefore, we obtain an isomorphism of $\mathcal{O}_{P_{n}}$-modules

$$
\begin{aligned}
\overline{\mathcal{P}}_{n}(\mathcal{J})=\overline{\mathcal{P}}(\mathcal{J}) / p^{n} \overline{\mathcal{P}}(\mathcal{J}) & \simeq \lim _{\vec{i}}\left(p^{i} \mathcal{P}(\mathcal{J}) / p^{n+i} \mathcal{P}(\mathcal{J})\right) \\
& \simeq \lim _{\vec{i}}\left(p^{i} \mathcal{P}_{n+i}(\mathcal{J})\right) .
\end{aligned}
$$

As the $\mathcal{O}_{P_{n+i}}$-algebra $\mathcal{P}_{n+i}(\mathcal{J})$ is quasi-coherent, $p^{i} \mathcal{P}_{n+i}(\mathcal{J})$ is a quasi-coherent $\mathcal{O}_{P_{n}}$-module. Taking the direct limit of these for variable $i$, it follows that $\overline{\mathcal{P}}_{n}(\mathcal{J})$ is quasi-coherent over $\mathcal{O}_{P_{n}}$.

Let $V \subset U$ be two affine open subsets of $P$. Using the flatness of $\Gamma\left(\mathbb{V}, \mathcal{O}_{\mathbb{P}}\right)$ over $\Gamma\left(\cup, \mathcal{O}_{\mathbb{P}}\right)$, we deduce from (4.2.1) that the canonical morphisms

$$
\begin{aligned}
& \Gamma\left(\mathbb{V}, \mathcal{O}_{\mathrm{P}}\right) \otimes_{\Gamma\left(\mathrm{U}, \mathcal{O}_{\mathrm{P}}\right)} \Gamma(\mathbb{U}, \mathcal{P}(\mathcal{J})) \rightarrow \Gamma(\mathbb{V}, \mathcal{P}(\mathcal{J})), \\
& \Gamma\left(\mathbb{V}, \mathcal{O}_{\mathbb{P}}\right) \otimes_{\Gamma\left(\mathrm{U}, \mathcal{O}_{\mathrm{P}}\right)} \Gamma\left(\mathbb{U}, \mathcal{T}_{n}(\mathcal{J})\right) \rightarrow \Gamma\left(\mathbb{V}, \mathcal{T}_{n}(\mathcal{J})\right)
\end{aligned}
$$

are isomorphisms. Therefore, $\mathcal{T}_{n}(\mathcal{J})$ is quasi-coherent over $\mathcal{O}_{P_{n}}$.

4.4. We now fix a notation. Let $A$ be a commutative ring, $\mathfrak{a} \subset A$ an ideal that contains $p$, and $m$ a positive integer. For any ideal $I \subset A$, we will denote by $I_{\mathfrak{a}}^{(m)}$ the ideal of $A$ generated by $\mathfrak{a}$ and the $p^{m}$-th powers of elements of $I$, or, equivalently, by $a$ and the $p^{m}$-th powers of a family of generators of $I$. When $\mathfrak{a}=p A$, we will simply use the notation $I^{(m)}$. Note that

$$
I_{\mathfrak{a}}^{(m)}=I^{(m)}+\mathfrak{a} .
$$


We extend this definition in an obvious way to sheaves of rings on topological spaces.

As in 4.1 , let $\mathbb{P}$ be a smooth formal scheme, and let $X \hookrightarrow P$ be a closed subscheme of its special fibre, defined by an ideal $\mathcal{J} \subset \mathcal{O}_{\mathrm{p}}$. We apply the previous definition to $\mathcal{J}$, using the ideal $\mathfrak{a}=p \mathcal{O}_{\mathrm{p}} \subset \mathcal{O}_{\mathrm{p}}$. We obtain in this way coherent ideals $\mathcal{J}^{(m)} \subset \mathcal{O}_{\mathrm{p}}$.

As $\mathcal{J}^{(m+1)} \subset \mathcal{J}^{(m)}$ for all $m \geq 0$, the $\mathcal{O}_{\mathrm{P}}$-algebras $\widehat{\mathcal{P}}\left(\mathcal{J}^{(m)}\right)$ and $\widetilde{\mathcal{P}}\left(\mathcal{J}^{(m)}\right)$ defined in 4.2 sit in inverse systems

$$
\begin{aligned}
& \cdots \rightarrow \widehat{\mathcal{P}}\left(\mathcal{J}^{(m+1)}\right) \rightarrow \widehat{\mathcal{P}}\left(\mathcal{J}^{(m)}\right) \rightarrow \cdots \rightarrow \widehat{\mathcal{P}}\left(\mathcal{J}^{(0)}\right), \\
& \cdots \rightarrow \widetilde{\mathcal{P}}\left(\mathcal{J}^{(m+1)}\right) \rightarrow \widetilde{\mathcal{P}}\left(\mathcal{J}^{(m)}\right) \rightarrow \cdots \rightarrow \widetilde{\mathcal{P}}\left(\mathcal{J}^{(0)}\right) .
\end{aligned}
$$

The following proposition is the key to comparisons between crystalline and rigid cohomologies in the proper singular case (see also [10, Section 2]):

PRoposition 4.5. With the previous notation, there exists functorial horizontal isomorphisms

$$
\mathcal{A}_{X, \mathrm{P}} \stackrel{\sim}{\longrightarrow} \lim _{\overleftarrow{m}}\left(\widehat{\mathcal{P}}\left(\mathcal{J}^{(m)}\right)_{K}\right) \stackrel{\sim}{\longrightarrow} \lim _{m}\left(\widetilde{\mathcal{P}}\left(\mathcal{J}^{(m)}\right)_{K}\right)
$$

Moreover, for any coherent ideal $\mathcal{K} \subset \mathcal{O}_{\mathbb{P}}$,

$$
R^{i} \lim _{m}\left(\widehat{\mathcal{P}}\left(\mathcal{J}^{(m)}+\mathcal{K}\right)_{K}\right)=R_{\overleftarrow{m}}^{i} \lim _{\overleftarrow{m}}\left(\widetilde{\mathcal{P}}\left(\mathcal{J}^{(m)}+\mathcal{K}\right)_{K}\right)=0
$$

for all $i \geq 1$.

Proof. For all $m \geq 0$, let $\eta_{m}=p^{-1 / p^{m}}$. We recall that the closed tube $[X]_{P, \eta_{m}}$ is defined as the open subset of $\mathbb{P}_{K}$ characterized by

$$
[X]_{\mathbb{P}, \eta_{m}} \cap \cup_{K}=\left\{x \in \mathbb{U}_{K}|\forall i,| f_{i}(x) \mid \leq \eta_{m}\right\}
$$

for any affine open subset $U \subset \mathbb{P}$ and any family of generators $\left(f_{i}\right)$ of $\mathcal{J}$ over $U$. Then $] X\left[_{\mathrm{P}}=\cup_{m}[X]_{\mathrm{P}, \eta_{m}}\right.$ is an admissible covering of $] X\left[_{\mathrm{P}}\right.$ by an increasing family of open subsets [2,1.1.9]. It follows that, for any open subset $U \subset \mathbb{P}$, the algebra of analytic functions on $] X\left[\mathrm{P} \cap \mathrm{U}_{K}\right.$ is defined by

$$
\Gamma(] X\left[\mathrm{p} \cap \mathbb{U}_{K}, \mathcal{O}_{] X\left[_{\mathrm{p}}\right.}\right)=\lim _{\overleftarrow{m}} \Gamma\left([X]_{\mathrm{P}, \eta_{m}} \cap \mathbb{U}_{K}, \mathcal{O}_{] X\left[_{\mathrm{p}}\right.}\right)
$$

Let $\mathcal{A}_{X, \mathbb{P}}^{(m)}=\mathrm{sp}_{*}\left(\mathcal{O}_{[X]_{\mathrm{P}, \eta_{m}}}\right)$. Then we obtain

$$
\mathcal{A}_{X, \mathrm{P}} \stackrel{\sim}{\sim} \lim _{\overleftarrow{m}} \mathcal{A}_{X, \mathrm{P}}^{(m)}
$$


We now define canonical morphisms

$$
\cdots \rightarrow \widehat{\mathcal{P}}\left(\mathcal{J}^{(m)}\right)_{K} \rightarrow \widetilde{\mathcal{P}}\left(\mathcal{J}^{(m)}\right)_{K} \rightarrow \mathcal{A}_{X, \mathrm{P}}^{(m)} \rightarrow \widehat{\mathcal{P}}\left(\mathcal{J}^{(m-1)}\right)_{K} \rightarrow \cdots
$$

such that the composition of any three consecutive morphisms is the transition morphism in the corresponding inverse system. The first one is simply defined by the canonical map $\widehat{\mathcal{P}}\left(\mathcal{J}^{(m)}\right) \rightarrow \widetilde{\mathcal{P}}\left(\mathcal{J}^{(m)}\right)$.

To define the two other ones, we work on affine open subsets and glue the local constructions. If $U=\operatorname{Spf} A \subset \mathbb{P}$, then the above description of $[X]_{\mathbb{P}, \eta_{m}} \cap \mathbb{U}_{K}$ shows that this is an affinoid subset of $\mathbb{U}_{K}$. From the definition of rigid analytic functions on such an affinoid, we obtain

$$
\begin{aligned}
\Gamma\left(\mathbb{U}, \mathcal{A}_{X, \mathrm{P}}^{(m)}\right) & =\Gamma\left([X]_{\mathrm{P}, \eta_{m}} \cap \mathbb{U}_{K}, \mathcal{O}_{\mathrm{P}_{K}}\right) \\
& =A_{K}\left\{T_{1}, \ldots, T_{r}\right\} /\left(f_{1}^{p^{m}}-p T_{1}, \ldots, f_{r}^{p^{m}}-p T_{r}\right),
\end{aligned}
$$

where $f_{1}, \ldots, f_{r}$ are generators of $\Gamma(\mathcal{U}, \mathcal{J}), T_{1}, \ldots, T_{r}$ is a family of indeterminates, and $A_{K}\left\{T_{1}, \ldots, T_{r}\right\} \subset A_{K}\left[\left[T_{1}, \ldots, T_{r}\right]\right]$ is the subring of formal power series the coefficients of which tend to 0 . Since $\mathcal{A}_{X, \mathrm{P}}^{(m)}$ is a Q-algebra, the morphism $\mathcal{O}_{\mathrm{P}} \rightarrow \mathcal{A}_{X, \mathrm{P}}^{(m)}$ factors uniquely through a morphism $\mathcal{P}\left(\mathcal{J}^{(m)}\right) / \mathcal{T}\left(\mathcal{J}^{(m)}\right) \rightarrow \mathcal{A}_{X, \mathrm{P}}^{(m)}$, and we obtain ring homomorphisms

$$
\Gamma\left(\mathbb{U}, \mathcal{P}\left(\mathcal{J}^{(m)}\right)\right) \rightarrow \Gamma\left(\mathbb{U}, \overline{\mathcal{P}}\left(\mathcal{J}^{(m)}\right)\right) \stackrel{\lambda}{\rightarrow} \Gamma\left(\mathbb{U}, \mathcal{A}_{X, \mathrm{P}}^{(m)}\right) .
$$

For $i \geq 1$, the composed homomorphism maps a section $\left(f_{j}^{p^{m}}\right)^{[i]}$ to $f_{j}^{p^{m} i} / i !=\left(p^{i} / i !\right) T_{j}^{i}$. On the other hand, (4.2.1) shows that $\Gamma\left(\mathbb{U}, \mathcal{P}\left(\mathcal{J}^{(m)}\right)\right)$ is generated as a $\Gamma\left(\mathbb{U}, \mathcal{O}_{\mathrm{P}}\right)$-module by the products $\left(f_{1}^{p^{m}}\right)^{\left[i_{1}\right]} \cdots\left(f_{r}^{p^{m}}\right)^{\left[i_{r}\right]}$, and the first homomorphism is surjective, since Lemma 4.3 (ii) and the quasi-compacity of $\mathbb{U}$ imply that $H^{1}\left(\mathbb{U}, \mathcal{T}\left(\mathcal{J}^{(m)}\right)\right)=0$. As $p^{i} / i ! \in Z_{p}$, it follows that $\lambda\left(\Gamma\left(U, \overline{\mathcal{P}}\left(\mathcal{J}^{(m)}\right)\right)\right)$ sits inside the image of $A\left\{T_{1}, \ldots, T_{r}\right\}$ in $\Gamma\left(\mathbb{U}, \mathcal{A}_{X, \mathrm{P}}^{(m)}\right)$. This implies that $\lambda$ is a continuous morphism when the source is endowed with the $p$-adic topology and the target with its Tate algebra topology. Therefore $\lambda$ factorizes uniquely through the $p$-adic completion of $\Gamma\left(\mathbb{U}, \overline{\mathcal{P}}\left(\mathcal{J}^{(m)}\right)\right)$.

We observe here that the natural map

$$
\Gamma\left(\mathbb{U}, \overline{\mathcal{P}}\left(\mathcal{J}^{(m)}\right)\right) / p^{n} \Gamma\left(\bigcup, \overline{\mathcal{P}}\left(\mathcal{J}^{(m)}\right)\right) \rightarrow \Gamma\left(\bigcup, \overline{\mathcal{P}}\left(\mathcal{J}^{(m)}\right) / p^{n} \overline{\mathcal{P}}\left(\mathcal{J}^{(m)}\right)\right)
$$

is an isomorphism for all $n$ : since $\overline{\mathcal{P}}\left(\mathcal{J}^{(m)}\right)$ is $p$-torsion free, it suffices to show that $H^{1}\left(\mathbb{U}, \overline{\mathcal{P}}\left(\mathcal{J}^{(m)}\right)\right)=0$. But $H^{1}\left(\mathbb{U}, \mathcal{P}\left(\mathcal{J}^{(m)}\right)\right)=0$, because (4.2.1) implies that $\mathcal{P}\left(\mathcal{J}^{(m)}\right)$ is a direct limit of coherent $\mathcal{O}_{\mathrm{p}}$-modules, and $H^{2}\left(\mathbb{U}, \mathcal{T}\left(\mathcal{J}^{(m)}\right)\right)=0$, because Lemma 4.3 (ii) shows that $\mathcal{T}\left(\mathcal{J}^{(m)}\right)$ is a direct limit of quasi-coherent $\mathcal{O}_{P_{n}}$-modules. So the $p$-adic completion of 
$\Gamma\left(\mathbb{U}, \overline{\mathcal{P}}\left(\mathcal{J}^{(m)}\right)\right)$ is isomorphic to $\Gamma\left(\mathbb{U}, \widetilde{\mathcal{P}}\left(\mathcal{J}^{(m)}\right)\right)$, and the factorization of $\lambda$ provides the morphism $\Gamma\left(\mathbb{U}, \widetilde{\mathcal{P}}\left(\mathcal{J}^{(m)}\right)_{K}\right) \rightarrow \Gamma\left(\mathbb{U}, \mathcal{A}_{X, \mathrm{P}}^{(m)}\right)$.

Finally, one can define a morphism

$$
A\left[T_{1}, \ldots, T_{r}\right] /\left(f_{1}^{p^{m}}-p T_{1}, \ldots, f_{r}^{p^{m}}-p T_{r}\right) \rightarrow \Gamma\left(\mathbb{U}, \mathcal{P}\left(\mathcal{J}^{(m-1)}\right)\right)
$$

by sending $T_{j}$ to $(p-1) !\left(f_{j}^{p^{m-1}}\right)^{[p]}$. Factorizing through the $p$-adic completions and tensorizing with $K$ provides the morphism $\Gamma\left(\mathbb{U}, \mathcal{A}_{X, \mathrm{P}}^{(m)}\right)$ $\rightarrow \Gamma\left(\mathbb{U}, \widehat{\mathcal{P}}\left(\mathcal{J}^{(m-1)}\right)_{K}\right)$, which is also unique.

Note that all these morphisms are horizontal, since they are continuous and induce the identity map on $\mathcal{O}_{\mathrm{P}, K}$, which is dense in every algebra under consideration.

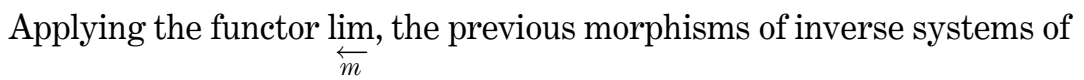
sheaves yield isomorphisms

$$
\lim _{\overleftarrow{m}}\left(\widehat{\mathcal{P}}\left(\mathcal{J}^{(m)}\right)_{K}\right) \simeq \lim _{\overleftarrow{m}}\left(\widetilde{\mathcal{P}}\left(\mathcal{J}^{(m)}\right)_{K}\right) \simeq \lim _{\overleftarrow{m}} \mathcal{A}_{X, \mathrm{P}}^{(m)} \simeq \mathcal{A}_{X, \mathrm{P}}
$$

To prove the second part of the proposition, it suffices to show that $R^{i} \lim _{\overleftarrow{m}}\left(\widehat{\mathcal{P}}\left(\mathcal{J}^{(m)}+\mathcal{K}\right)_{K}\right)=0$ for $i \geq 1$. Observe first that, for any affine open subset $U \subset \mathbb{P}$,

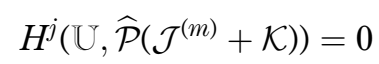

for $j \geq 1$, thanks to Lemma 4.3 and to the algebraic Mittag-Leffler criterion. As $\mathbb{U}$ is noetherian, taking sections on $\mathbb{U}$ commutes with tensorisation with $K$, and it follows that

$$
H^{j}\left(\mathbb{U}, \widehat{\mathcal{P}}\left(\mathcal{J}^{(m)}+\mathcal{K}\right)_{K}\right)=0
$$

for $j \geq 1$. On the other hand, $\Gamma\left(\mathbb{U}, \mathcal{P}\left(\mathcal{J}^{(m)}+\mathcal{K}\right)\right)_{K}=\Gamma\left(\mathbb{U}, \mathcal{O}_{\mathbb{P}}\right)_{K}$ for any $m \geq 0$, so that the image of $\Gamma\left(\mathbb{U}, \mathcal{O}_{\mathbb{P}}\right)_{K}$ in $\left(\Gamma\left(\mathbb{U}, \mathcal{P}\left(\mathcal{J}^{(m)}+\mathcal{K}\right)\right)^{\wedge}\right)_{K}$ is dense for the $p$-adic topology. Lemma 4.3 implies that $\Gamma\left(\mathbb{U}, \mathcal{P}\left(\mathcal{J}^{(m)}+\mathcal{K}\right)\right)^{\wedge}$ $=\Gamma\left(\mathbb{U}, \widehat{\mathcal{P}}\left(\mathcal{J}^{(m)}+\mathcal{K}\right)\right)$, hence it follows that, for $m^{\prime} \geq m$, the image of

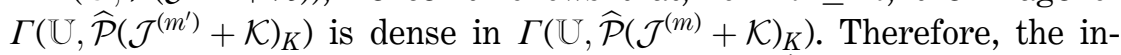
verse system of topological vector spaces $\left(\Gamma\left(\mathbb{U}, \widehat{\mathcal{P}}\left(\mathcal{J}^{(m)}+\mathcal{K}\right)_{K}\right)\right)_{m \geq 0}$ satifies the topological Mittag-Leffler condition ( $\left.\mathrm{ML}^{\prime}\right)$ [12, $0_{\mathrm{III}}, 13.2 .4$ (i)]. It is easy to see that, together with (4.5.4), this property implies that the inverse system $\left(\widehat{\mathcal{P}}\left(\mathcal{J}^{(m)}+\mathcal{K}\right)_{K}\right)_{m \geq 0}$ is lim-acyclic.

CoRollary 4.6. Let $X$ be a proper $k$-scheme, and let $X \hookrightarrow \mathbb{P}$ be a closed immersion into a smooth formal scheme over $W$. There exists 
canonical isomorphisms

(4.6.1) $\quad \underline{\mathrm{R}}_{\text {rig }}(X / K) \stackrel{\sim}{\longrightarrow} \lim _{\frac{\leftarrow}{}}\left(\widehat{\mathcal{P}}\left(\mathcal{J}^{(m)}\right)_{K}\right) \otimes_{\mathcal{O}_{\mathrm{P}}} \Omega_{\mathrm{P}}^{\bullet}$

$\stackrel{\sim}{\longrightarrow} \lim _{m}\left(\widetilde{\mathcal{P}}\left(\mathcal{J}^{(m)}\right)_{K}\right) \otimes_{\mathcal{O}_{\mathbb{P}}} \Omega_{\mathbb{P}}^{\bullet}$

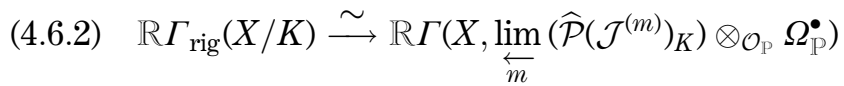

$$
\stackrel{\sim}{\longrightarrow} \Gamma\left(X, \underset{\overleftarrow{m}}{\lim }\left(\widetilde{\mathcal{P}}\left(\mathcal{J}^{(m)}\right)_{K}\right) \otimes_{\mathcal{O}_{\mathbb{P}}} \Omega_{\mathrm{P}}^{\bullet}\right)
$$

functorial with respect to the immersion $X \hookrightarrow \mathbb{P}$.

Proof. Thanks to the previous proposition, this follows immediately from (4.1.3) and (4.1.4).

We now observe that Proposition 4.5 allows to write the rigid cohomology of a proper scheme as the inverse limit of the crystalline cohomologies of its infinitesimal neighbourhoods in a smooth scheme (tensorized by $K$ ).

THEOREM 4.7. Let $Y$ be a smooth $k$-scheme, and let $X \hookrightarrow Y$ be a closed subscheme that is proper over $k$. Let $\mathcal{I} \subset \mathcal{O}_{Y}$ be the ideal of $X$ in $Y$, and let $X^{(m)} \subset Y$ be the closed subscheme defined by $\mathcal{I}^{(m)}$ (as defined in 4.4). There exists functorial isomorphisms

$$
\begin{aligned}
& \mathbb{R} \underline{\Gamma}_{\text {rig }}(X / K) \stackrel{\sim}{\longrightarrow} \underset{\overleftarrow{m}}{\lim }\left(\left(\mathbb{R} u_{X^{(m)} / W *} \mathcal{O}_{X^{(m)} / W}\right)_{K}\right) \\
& \mathbb{R} \Gamma_{\text {rig }}(X / K) \stackrel{\sim}{\longrightarrow} \underset{\leftarrow}{\lim }\left(\mathbb{R} \Gamma_{\text {crys }}\left(X^{(m)} / W, \mathcal{O}_{X^{(m)} / W}\right)_{K}\right)
\end{aligned}
$$

in $D^{\mathrm{b}}(X, K)$ and $D^{\mathrm{b}}(K)$ respectively.

Proof. For $m \leq m^{\prime}$, let $i_{\text {crys }}^{\left(m^{\prime}, m\right)}:\left(X^{(m)} / W\right)_{\text {crys }} \rightarrow\left(X^{\left(m^{\prime}\right)} / W\right)_{\text {crys }}$ denote the morphism of topos induced by the inclusion $X^{(m)} \hookrightarrow X^{\left(m^{\prime}\right)}$. To define the right hand side of (4.7.1) and (4.7.2), one can work with the category of inverse systems $\left(E^{(m)}, \rho^{(m)}\right)$, where $E^{(m)}$ is a sheaf on the site $\operatorname{Crys}\left(X^{(m)} / W\right)$ and $\rho^{(m)}$ is a morphism of sheaves $\left(i_{\text {crys }}^{(m, m-1)}\right)^{-1} E^{(m)} \rightarrow E^{(m-1)}$. These systems form a topos $\left(X^{(\bullet)} / W\right)_{\text {crys }}$, and the family of structural sheaves $\mathcal{O}_{X^{(m)} / W}$, together with the functoriality morphisms, is an object $\mathcal{O}_{X^{(\bullet)} / \mathrm{w}}$ in this topos. 
Using the fact that $\left|X^{(m)}\right|=|X|$ for all $m$, the family of functors $u_{X^{(m)} / W *}$ defines a functor $u_{X^{(\bullet)} / W *}$ from $\left(X^{(\bullet)} / W\right)_{\text {crys }}$ to the topos $X_{\mathrm{Zar}}^{(\bullet)}$ of inverse systems of sheaves on $X$. We denote by $\mathbb{R} \lim _{\overleftarrow{m}}\left(\left(\mathbb{R} u_{X^{(m)} / W *} \mathcal{O}_{X^{(m)} / W}\right)_{K}\right)$ the complex obtained by applying to $\mathcal{O}_{X(\bullet)} / \mathrm{W}$ the right derived functor of $u_{X^{(\bullet)} / W *}$, tensorizing with $K$, and applying the $\underset{\overleftarrow{m}}{\mathbb{R}} \lim _{\overleftarrow{m}}$ functor. The complex $\mathbb{R} \lim _{\overleftarrow{m}}\left(\mathbb{R} \Gamma_{\text {crys }}\left(X^{(m)} / W, \mathcal{O}_{X^{(m)} / W}\right)_{K}\right)$ is defined similarly.

Let us assume first that $Y$ can be embedded in a smooth formal scheme $\mathbb{P}$. Let $\mathcal{J}$ be the ideal of $X$ in $\mathrm{P}$, and let $\mathcal{K}$ be the ideal of $Y$ in $\mathrm{P}$. Then $X^{(m)}$ is the closed subscheme of $\mathbb{P}$ defined by the ideal $\mathcal{J}^{(m)}+\mathcal{K}$. Thanks to the functoriality properties of the linearization functor used in the construction of the comparison isomorphism between crystalline and de Rham cohomologies [7, 6.9], the family of linearizations of $\Omega_{\mathrm{p}}^{\bullet}$ on the sites $\operatorname{Crys}\left(X^{(m)} / W\right)$ provides a resolution of $\mathcal{O}_{X^{(\bullet)} / W}$ that is acyclic for the functor $u_{X \bullet / W *}$. This defines an isomorphism

$$
\mathbb{R} u_{X^{(\bullet)} / W *}\left(\mathcal{O}_{X^{(\bullet)} / \mathbb{W}}\right) \stackrel{\sim}{\longrightarrow} \widehat{\mathcal{P}}\left(\mathcal{J}^{(\bullet)}+\mathcal{K}\right) \otimes \Omega_{\mathrm{P}}^{\bullet}
$$

in the derived category of inverse systems of $W$-modules on $X$, which has the isomorphism (3.1.2) as component of index $m$ for each $m$. Tensorizing with $K$, applying $\underset{\overleftarrow{m}}{\lim }$, and taking (4.5.2) into account, one gets an isomorphism

$$
\mathbb{R} \lim _{\overleftarrow{m}}\left(\left(\mathbb{R} u_{X^{(m)} / W *} \mathcal{O}_{X^{(m)} / W}\right)_{K}\right) \stackrel{\sim}{\longrightarrow} \lim _{\overleftarrow{m}}\left(\widehat{\mathcal{P}}\left(\mathcal{J}^{(m)}+\mathcal{K}\right)_{K}\right) \otimes \Omega_{\mathbb{P}}^{\bullet}
$$

As

$$
\mathbb{R} \underline{\Gamma}_{\text {rig }}(X / K) \stackrel{\sim}{\longrightarrow} \lim _{\overleftarrow{m}}\left(\widehat{\mathcal{P}}\left(\mathcal{J}^{(m)}\right)_{K}\right) \otimes \Omega_{\mathrm{P}}^{\bullet}
$$

by (4.6.1), it suffices to prove that the functoriality morphism

$$
\lim _{\overleftarrow{m}}\left(\widehat{\mathcal{P}}\left(\mathcal{J}^{(m)}\right)_{K}\right) \otimes \Omega_{\mathbb{P}}^{\bullet} \longrightarrow \lim _{\overleftarrow{m}}\left(\widehat{\mathcal{P}}\left(\mathcal{J}^{(m)}+\mathcal{K}\right)_{K}\right) \otimes \Omega_{\mathbb{P}}^{\bullet}
$$

is a quasi-isomorphism.

This is a local statement on $X$, so we may assume that $\mathbb{P}, Y$, and $X$ are affine. It is independent of the choice of the embedding $\mathbb{P}$ of $Y$, because it is functorial with respect to $P$, so the morphisms defined by any two embeddings $\mathbb{P}, \mathbb{P}^{\prime}$ can be compared via the diagonal embedding into $\mathbb{P} \times_{W} \mathbb{P}^{\prime}$. But the left hand side of (4.7.4) is independent of $P$ up to quasi-isomorphism because it computes canonically $\mathbb{R} \underline{\Gamma_{\text {rig }}}(X / K)$, and so does the right hand 
side because, for each $m$, it computes canonically $\left(\mathbb{R} u_{X^{(m)} / W *} \mathcal{O}_{X^{(m)} / W}\right)_{K}$ and it has lim-acyclic terms. This allows to reduce to the case where $P$ is a smooth lifting of $Y$. Then the source and target of (4.7.4) are identical, and the morphism is the identity.

This defines the isomorphism (4.7.1) when $Y$ can be embedded into a smooth formal scheme over $W$. In the general case, one chooses an affine open covering $\mathfrak{l}=\left(U_{\alpha}\right)$ of $Y$ and closed immersions $U_{\alpha} \hookrightarrow \mathbb{P}_{\alpha}$ into smooth formal schemes over $W$. Then one can use for each $X^{(m)}$ the Čech resolution introduced in 3.1 to compute $\mathbb{R} \lim _{\overleftarrow{m}}\left(\left(\mathbb{R} u_{X^{(m)} / W *} \mathcal{O}_{X^{(m)} / W}\right)_{K}\right)$ and the Čech resolution introduced in 4.1 to define $\mathbb{R} \underline{\Gamma}_{\text {rig }}(X / K)$. The functoriality of the previous construction provides a morphism between these Čech resolutions, and this morphism is a quasi-isomorphism because the corresponding morphism for each intersection $U_{\underline{\alpha}}$ of the covering is a quasiisomorphism.

The isomorphism (4.7.2) follows from (4.7.1) by taking global sections and using the commutations between $\mathbb{R} \lim$ and $\mathbb{R} \Gamma(X,-)$, and between $\mathbb{R} \Gamma(X,-)$ and tensorisation by $K$.

REMARK 4.8. As $Y$ is noetherian, the ind-scheme $\left(X^{(m)}\right)_{m \geq 0}$ is isomorphic to the ind-scheme that consists in all infinitesimal neighbourhoods $X_{n}$ of $X$ in $Y$. Therefore, one can rewrite isomorphisms (4.7.1) and (4.7.2) as

$$
\begin{aligned}
& \mathbb{R} \underline{\Gamma}_{\text {rig }}(X / K) \stackrel{\sim}{\longrightarrow} \mathbb{R} \lim _{\overleftarrow{n}}\left(\left(\mathbb{R} u_{X_{n} / W *} \mathcal{O}_{X_{n} / W}\right)_{K}\right), \\
& \mathbb{R} \Gamma_{\text {rig }}(X / K) \stackrel{\sim}{\longrightarrow} \mathbb{R} \lim _{\overleftarrow{n}}\left(\mathbb{R} \Gamma_{\text {crys }}\left(X_{n} / W, \mathcal{O}_{X_{n} / W}\right)_{K}\right)
\end{aligned}
$$

\section{The comparison theorem}

Given a proper $k$-scheme $X$ enbedded as a closed subscheme in a smooth $k$-scheme $Y$, we construct now a de Rham-Witt complex on $Y$ with coefficients supported in $X$, and we prove that its cohomology is canonically isomorphic to the rigid cohomology of $X$. This de Rham Witt complex can easily be defined using divided power envelopes for the ideals of sections of $W_{n} \mathcal{O}_{Y}$ that vanish on the infinitesimal neighbourhoods $X^{(m)}$ of $X$ in $Y$. However, because of the flatness over $W_{n}$ required in Theorem 3.6, we need to use also an alternate definition based on $p$-torsion free quotients of PD-envelopes, analogous to the construction introduced in the previous section. 
More generally, we prove a similar comparison theorem for the rigid cohomology with compact supports of a separated $k$-scheme of finite type.

5.1. Let $Y$ be a smooth $k$-scheme of finite type, and let $i: X \hookrightarrow Y$ be the inclusion of a closed subscheme defined by an ideal $\mathcal{I}_{Y} \subset \mathcal{O}_{Y}$. For each PD-thickening $(U, T, \delta)$ of an open subset $U$ of $Y$, we will denote by $\mathcal{K}_{T}$ the ideal of $U$ in $T$ and by $\mathcal{I}_{T}$ the ideal of $X \cap U$ in $T$.

For each $m \geq 0$, let $i^{(m)}: X^{(m)} \hookrightarrow Y$ be the inclusion of the closed subscheme defined by $\mathcal{I}_{Y}^{(m)}$, where $\mathcal{I}_{Y}^{(m)}$ is defined as in 4.4. The ideal of $X^{(m)}$ in $T$ is then equal to

$$
\left(\mathcal{I}_{T}\right)_{\mathcal{K}_{T}}^{(m)}:=\left(f^{p^{m}}\right)_{f \in \mathcal{I}_{T}}+\mathcal{K}_{T}
$$

We will work with the crystalline direct images $i_{\text {crys } *}^{(m)} \mathcal{O}_{X^{(m)} / W}$. As recalled in 3.9, these are crystals on $Y / W$, which can be described as follows: for each $(U, T, \delta)$ as above, the evaluation on $T$ of $i_{\text {crys } *}^{(m)} \mathcal{O}_{X^{(m)} / W}$ is given by

$$
\left(i_{\text {crys } *}^{(m)} \mathcal{O}_{X^{(m)} / W}\right)_{T}=\mathcal{P}_{\delta}\left(\left(\mathcal{I}_{T}\right)_{\mathcal{K}_{T}}^{(m)}\right),
$$

where $\mathcal{P}_{\delta}$ denotes the divided power envelope with compatiblity with the divided powers $\delta$ on $\mathcal{K}_{T}$. When $(U, T, \delta)$ varies, the transition maps are defined by the functoriality of divided power envelopes.

To simplify the notation, we set $\mathcal{P}_{X, Y}^{(m)}=i_{\text {crys }}^{(m)} \mathcal{O}_{X^{(m)} / W}$. For $m \geq 1$, the inclusions $X^{(m-1)} \hookrightarrow X^{(m)}$ define functoriality morphisms

$$
\rho^{(m)}: \mathcal{P}_{X, Y}^{(m)} \longrightarrow \mathcal{P}_{X, Y}^{(m-1)},
$$

which turn the family $\left(\mathcal{P}_{X, Y}^{(m)}\right)_{m \geq 0}$ into an inverse system of crystals. As in 2.1, we can take the evaluations of these crystals on the thickenings $Y \hookrightarrow W_{n} Y$, and let $n, m$ vary. In this way, we obtain an inverse system of $W_{n} \mathcal{O}_{Y}$-modules $\left(\mathcal{P}_{X, Y, n}^{(m) W}\right)_{m, n}$ and an inverse system of complexes $\left(\mathcal{P}_{X, Y, n}^{(m) W} \otimes_{W_{n} \mathcal{O}_{Y}} W_{n} \Omega_{Y}^{\bullet}\right)_{m, n}$. We define

$$
\begin{aligned}
& \widehat{\mathcal{P}}_{X, Y}^{(m) W}:=\lim _{\overleftarrow{n}} \mathcal{P}_{X, Y, n}^{(m) W}, \quad \mathcal{A}_{X, Y}^{W}:=\lim _{\overleftarrow{m}}\left(\widehat{\mathcal{P}}_{X, Y}^{(m) W} \otimes K\right), \\
& \widehat{\mathcal{P}}_{X, Y}^{(m) W} \widehat{\otimes}_{W \mathcal{O}_{Y}} W \Omega_{Y}^{\bullet}:=\lim _{\overleftarrow{n}}\left(\mathcal{P}_{X, Y, n}^{(m) W} \otimes_{W_{n} \mathcal{O}_{Y}} W_{n} \Omega_{Y}^{\bullet}\right), \\
& \mathcal{A}_{X, Y}^{W} \widehat{\otimes}_{W \mathcal{O}_{Y}} W \Omega_{Y}^{\bullet}:=\lim _{\overleftarrow{m}}\left(\left(\widehat{\mathcal{P}}_{X, Y}^{(m) W} \widehat{\otimes}_{W \mathcal{O}_{Y}} W \Omega_{Y}^{\bullet}\right) \otimes K\right)
\end{aligned}
$$


As $W_{n} \Omega_{Y}^{0}=W_{n} \mathcal{O}_{Y}$, we get in degree 0

$$
\begin{aligned}
& \widehat{\mathcal{P}}_{X, Y}^{(m) W} \widehat{\otimes}_{W \mathcal{O}_{Y}} W \Omega_{Y}^{0}=\lim _{\overleftarrow{n}}\left(\mathcal{P}_{X, Y, n}^{(m) W} \otimes_{W_{n} \mathcal{O}_{Y}} W_{n} \Omega_{Y}^{0}\right) \simeq \widehat{\mathcal{P}}_{X, Y}^{(m) W}, \\
& \mathcal{A}_{X, Y}^{W} \widehat{\otimes}_{W \mathcal{O}_{Y}} W \Omega_{Y}^{0}=\lim _{\overleftarrow{m}}\left(\left(\widehat{\mathcal{P}}_{X, Y}^{(m) W} \widehat{\otimes}_{W \mathcal{O}_{Y}} W \Omega_{Y}^{0}\right) \otimes K\right) \simeq \mathcal{A}_{X, Y}^{W} .
\end{aligned}
$$

We will denote the transition morphisms by

$$
\begin{aligned}
\hat{\rho}^{(m)}: \widehat{\mathcal{P}}_{X, Y}^{(m) W} & \rightarrow \widehat{\mathcal{P}}_{X, Y}^{(m-1) W}, \\
\hat{\rho}^{(m) \bullet}: \widehat{\mathcal{P}}_{X, Y}^{(m) W} \widehat{\otimes}_{W \mathcal{O}_{Y}} W \Omega_{Y}^{\bullet} & \rightarrow \widehat{\mathcal{P}}_{X, Y}^{(m-1) W} \widehat{\otimes}_{W \mathcal{O}_{Y}} W \Omega_{Y}^{\bullet} .
\end{aligned}
$$

Note that, since the crystals $\mathcal{P}_{X, Y}^{(m)}$ are supported in $X$, so are the complexes $\widehat{\mathcal{P}}_{X, Y}^{(m) W} \widehat{\otimes}_{W \mathcal{O}_{Y}} W \Omega_{Y}^{\bullet}$ and $\mathcal{A}_{X, Y}^{W} \widehat{\otimes}_{W \mathcal{O}_{Y}} W \Omega_{Y}^{\bullet}$. We can now state the main result of this article.

THeOREm 5.2. Let $X$ be a $k$-scheme of finite type, and let $i: X \hookrightarrow Y$ be a closed immersion into a smooth $k$-scheme.

(i) If there exists a closed immersion $Y \hookrightarrow \mathbb{P}$ of $Y$ into a smooth formal $W$-scheme $\mathbb{P}$ endowed with a lifting $F$ of the absolute Frobenius endomorphism, these data define a quasi-isomorphism of complexes of sheaves of $K$-vector spaces supported in $X$

$$
\mathcal{A}_{X, \mathrm{P}} \otimes_{\mathcal{O}_{\mathrm{P}}} \Omega_{\mathrm{P}}^{\bullet} \longrightarrow \mathcal{A}_{X, Y}^{W} \widehat{\otimes}_{W \mathcal{O}_{Y}} W \Omega_{Y}^{\bullet},
$$

functorial with respect to $(X, Y, \mathbb{P}, F)$. Via the identifications of $[4,1.5]$, its image in $D^{\mathrm{b}}(X, K)$ is independent of the choice of the embedding of $Y$ into $(\mathbb{P}, F)$.

(ii) Without extra assumption, there exists in $D^{\mathrm{b}}(X, K)$ a canonical isomorphism

$$
\mathbb{R} \underline{\Gamma}_{\text {rig }}(X / K) \stackrel{\sim}{\longrightarrow} \mathcal{A}_{X, Y}^{W} \widehat{\otimes}_{W \mathcal{O}_{Y}} W \Omega_{Y}^{\bullet},
$$

functorial with respect to the couple $(X, Y)$ and defined by (5.2.1) whenever the assumptions of (i) are satisfied.

(iii) If $X$ is proper, there exists in $D^{\mathrm{b}}(K)$ a canonical isomorphism

$$
\mathbb{R} \Gamma_{\text {rig }}(X / K) \stackrel{\sim}{\longrightarrow} \mathbb{R} \Gamma\left(X, \mathcal{A}_{X, Y}^{W} \widehat{\otimes}_{W \mathcal{O}_{Y}} W \Omega_{Y}^{\bullet}\right),
$$

functorial with respect to the couple $(X, Y)$.

The proof of the theorem will be given in subsection 5.7. It requires some additional constructions, which we detail in the next subsections. 
5.3. We first want to show that one can perform constructions similar to those of 5.1 using the $p$-torsion free envelopes defined in 4.4.

We begin with a local construction. Let $U \subset Y$ be an affine open subset, and let $U$ be a smooth affine formal scheme on $W$ lifting $U$; for all $n \geq 1$, let $U_{n}$ be the reduction of $U$ on $W_{n}$. We denote by $\mathcal{I} \subset \mathcal{O}_{U}$ the ideal defining $X \cap U$ in $U$, and we define $\mathcal{I}^{(m)}, \mathcal{P}\left(\mathcal{I}^{(m)}\right), \widehat{\mathcal{P}}\left(\mathcal{I}^{(m)}\right), \mathcal{T}\left(\mathcal{I}^{(m)}\right), \overline{\mathcal{P}}\left(\mathcal{I}^{(m)}\right)$ and $\widetilde{\mathcal{P}}\left(\mathcal{I}^{(m)}\right)$ as in 4.4. We set

$$
\begin{aligned}
& \mathcal{P}_{n}\left(\mathcal{I}^{(m)}\right)=\mathcal{P}\left(\mathcal{I}^{(m)}\right) / p^{n} \mathcal{P}\left(\mathcal{I}^{(m)}\right) \simeq \widehat{\mathcal{P}}\left(\mathcal{I}^{(m)}\right) / p^{n} \widehat{\mathcal{P}}\left(\mathcal{I}^{(m)}\right), \\
& \overline{\mathcal{P}}_{n}\left(\mathcal{I}^{(m)}\right)=\overline{\mathcal{P}}\left(\mathcal{I}^{(m)}\right) / p^{n} \overline{\mathcal{P}}\left(\mathcal{I}^{(m)}\right) \simeq \widetilde{\mathcal{P}}\left(\mathcal{I}^{(m)}\right) / p^{n} \widetilde{\mathcal{P}}\left(\mathcal{I}^{(m)}\right) .
\end{aligned}
$$

Let us recall how the crystal $\mathcal{P}_{X, Y}^{(m)}$ corresponds to the $\mathcal{O}_{U}$-module $\widehat{\mathcal{P}}\left(\mathcal{I}^{(m)}\right)$ in the classical equivalence between crystals on $\operatorname{Crys}(U / W)$ and $p$ adically complete $\mathcal{O}_{U}$-modules endowed with an integrable and topologically quasi-nilpotent connection. If $(V, T)$ is a thickening in $\operatorname{Crys}(U / W)$, there exists locally on $T$ an integer $n \geq 1$ such that $p^{n} \mathcal{O}_{T}=0$, and a $W$ morphism $h: T \rightarrow U_{n}$ extending the immersion $V \hookrightarrow U_{n}$. Then the $\mathcal{O}_{T^{-}}$ algebra $h^{*}\left(\mathcal{P}_{n}\left(\mathcal{I}^{(m)}\right)\right)$ does not depend on the choice of $n$ and $h$, up to a canonical isomorphism defined by the HPD-stratification of $\mathcal{P}_{n}\left(\mathcal{I}^{(m)}\right)$, and it can be identified with the Zariski sheaf $\left(\mathcal{P}_{X, Y}^{(m)}\right)_{T}$ on $T$ defined by the crystal $\mathcal{P}_{X, Y}^{(m)}$ [7, Th. 6.6 and claim p. 6.3].

As we already observed in 4.4, the quotient $\overline{\mathcal{P}}_{n}\left(\mathcal{I}^{(m)}\right)$ of $\mathcal{P}\left(\mathcal{I}^{(m)}\right)$ is endowed with the quotient connection. Since the connection of $\mathcal{P}_{n}\left(\mathcal{I}^{(m)}\right)$ is quasi-nilpotent and $\overline{\mathcal{P}}_{n}\left(\mathcal{I}^{(m)}\right)$ is a quotient of $\mathcal{P}_{n}\left(\mathcal{I}^{(m)}\right)$, the connection of $\overline{\mathcal{P}}_{n}\left(\mathcal{I}^{(m)}\right)$ is also quasi-nilpotent. It follows that $\overline{\mathcal{P}}_{n}\left(\mathcal{I}^{(m)}\right)$ defines by the previous construction a crystal in $\mathcal{O}_{U / W}$-algebras $\overline{\mathcal{P}}_{X \cap U, U}^{(m)}$, endowed with a surjective morphism $\pi^{(m)}: \mathcal{P}_{X \cap U, U}^{(m)} \rightarrow \overline{\mathcal{P}}_{X \cap U, U}^{(m)}$.

In order to be able to glue such local constructions and to define a crystal globally on $Y$, we need to show that the crystal $\overline{\mathcal{P}}_{X \cap U, U}^{(m)}$ does not depend, up to canonical isomorphism, on the choice of the lifting $U$. Let $U^{\prime}$ be a second lifting of $U$, defining a crystal $\overline{\mathcal{P}}_{X \cap U, U}^{\prime(m)}$ by the previous construction. As $U$ is affine, there exists a (non-canonical) $W$-isomorphism $u: U \stackrel{\sim}{\longrightarrow} U^{\prime}$ inducing the identity on $U$, hence on each $X^{(m)}$. If $\mathcal{I}^{\prime}$ denotes the ideal of $X$ in $\mathbb{U}^{\prime}$, the functoriality homomorphism $u^{*} \mathcal{P}\left(\mathcal{I}^{\prime(m)}\right) \rightarrow \mathcal{P}\left(\mathcal{I}^{(m)}\right)$ is then an isomorphism compatible with the connections. It induces an isomorphism $u^{*} \mathcal{T}\left(\mathcal{I}^{\prime(m)}\right) \stackrel{\sim}{\longrightarrow} \mathcal{T}\left(\mathcal{I}^{(m)}\right)$ between the torsion subsheaves, and we get a horizontal isomorphism

$$
\varepsilon_{u}: u^{*} \overline{\mathcal{P}}\left(\mathcal{I}^{\prime(m)}\right) \stackrel{\sim}{\longrightarrow} \overline{\mathcal{P}}\left(\mathcal{I}^{(m)}\right) .
$$

If $(V, T)$ is a thickening in $\operatorname{Crys}(U / W)$, if we choose a morphism 
$h: T \rightarrow U_{n}$ as above to define $\left(\overline{\mathcal{P}}_{X \cap U, U}^{(m)}\right)_{T}$, and if we use the composition $h^{\prime}=u_{n} \circ h: T \rightarrow U_{n}^{\prime}$ to define $\left(\overline{\mathcal{P}}_{X \cap U, U}^{\prime(m)}\right)_{T}$ (denoting by $u_{n}$ the reduction of $u$ modulo $p^{n}$ ), then the evaluation on $T$

$$
\varepsilon_{T}:\left(\overline{\mathcal{P}}_{X \cap U, U}^{\prime(m)}\right)_{T} \stackrel{\sim}{\longrightarrow}\left(\overline{\mathcal{P}}_{X \cap U, U}^{(m)}\right)_{T}
$$

of the wanted canonical isomorphism $\varepsilon: \overline{\mathcal{P}}_{X \cap U, U}^{\prime(m)} \stackrel{\sim}{\longrightarrow} \overline{\mathcal{P}}_{X \cap U, U}^{(m)}$ is defined to be the pullback of the reduction of $\varepsilon_{u} \bmod p^{n}$ by $h$. One observes that, if one changes the choices made for $h, h^{\prime}$, or $u$, then the pull-back of $\varepsilon_{u}$ is modified by the same isomorphisms deduced from the HPD-stratification that occur in the definition of the Zariski sheaves $\left(\overline{\mathcal{P}}_{X \cap U, U}^{(m)}\right)_{T}$ and $\left(\overline{\mathcal{P}}_{X \cap U, U}^{\prime(m)}\right)_{T}$, so that $\varepsilon_{T}$ finally does not depend on any choice. One also checks that, thanks to the cocycle property of the HPD-stratification, these isomorphisms satisfy the necessary transitivity properties. This allows to glue the local definitions on affine open subsets and to construct the crystal $\overline{\mathcal{P}}_{X, Y}^{(m)}$ globally on $Y$.

For $m \geq 1$, the natural homomorphisms $\overline{\mathcal{P}}\left(\mathcal{I}^{(m)}\right) \rightarrow \overline{\mathcal{P}}\left(\mathcal{I}^{(m-1)}\right)$ defined on smooth liftings of affine open subschemes of $Y$ can also be glued so as to define homomorphisms of crystals $\bar{\rho}^{(m)}: \overline{\mathcal{P}}_{X, Y}^{(m)} \rightarrow \overline{\mathcal{P}}_{X, Y}^{(m-1)}$ on $Y$, turning the family of crystals $\left(\overline{\mathcal{P}}_{X, Y}^{(m)}\right)_{m \geq 0}$ into an inverse system. On the other hand, we have by construction a surjective morphism of crystals $\pi^{(m)}: \mathcal{P}_{X, Y}^{(m)} \rightarrow \overline{\mathcal{P}}_{X, Y}^{(m)}$ for each $m$, and these define a morphism of inverse systems. The next lemma will allow us to replace, up to isogeny, the inverse system of crystals $\left(\mathcal{P}_{X, Y}^{(m)}\right)_{m \geq 0}$ by the inverse system $\left(\overline{\mathcal{P}}_{X, Y}^{(m)}\right)_{m \geq 0}$.

LEMMA 5.4. With the previous notation and hypotheses, let $m$ be fixed, and let $s \geq 0$ be an integer. If $s$ is big enough, there exists a unique morphism of crystals $\sigma_{s}^{(m)}: \overline{\mathcal{P}}_{X, Y}^{(m)} \rightarrow \mathcal{P}_{X, Y}^{(m-1)}$ such that

$$
\sigma_{s}^{(m)} \circ \pi^{(m)}=p^{s} \rho^{(m)}, \quad \pi^{(m-1)} \circ \sigma_{s}^{(m)}=p^{s} \bar{\rho}^{(m)} .
$$

Proof. The unicity claim follows from the fact that each $\pi^{(m)}$ is a epimorphism of crystals. Note also that, if there exists a couple $\left(s, \sigma_{s}^{(m)}\right)$ that satifies the conditions of the lemma, then, for any $t \geq 0$, the couple $\left(s+t, \sigma_{s+t}^{(m)}:=p^{t} \sigma_{s}^{(m)}\right)$ also satifies these conditions. As $Y$ is quasi-compact, this allows to check the lemma locally on $Y$.

So we may assume that $Y$ is affine and has a smooth formal lifting $Y$ on $W$. Let us keep the notation of 5.3, only replacing $U$ and $U$ by $Y$ and Y. Let $\left(f_{1}, \ldots, f_{r}\right)$ be a family of generators of $\mathcal{I}$, and let $\mathcal{R}=$ $\mathcal{O}_{Y}\left[T_{1}, \ldots, T_{r}\right]$. We define an $\mathcal{O}_{Y}$-algebra $\mathcal{A}^{(m)}\left(f_{1}, \ldots, f_{r}\right)$ (the completion of which is an integral model of the algebra $\mathcal{A}_{X, Y}^{(m)}$ used in the proof of 
Proposition 4.5) by setting

$$
\mathcal{A}^{(m)}\left(f_{1}, \ldots, f_{r}\right):=\mathcal{R} /\left(f_{1}^{p^{m}}-p T_{1}, \ldots, f_{r}^{p^{m}}-p T_{r}\right) .
$$

The canonical homomorphism $\mathcal{O}_{\mathrm{Y}} \rightarrow \mathcal{A}^{(m)}\left(f_{1}, \ldots, f_{r}\right)$ maps the ideal $\mathcal{I}^{(m)}$ to the PD-ideal $p \mathcal{A}^{(m)}\left(f_{1}, \ldots, f_{r}\right)$, hence it factorizes uniquely through a PDmorphism

$$
\alpha^{(m)}: \mathcal{P}\left(\mathcal{I}^{(m)}\right) \rightarrow \mathcal{A}^{(m)}\left(f_{1}, \ldots, f_{r}\right)
$$

On the other hand, there is a unique homomorphism of $\mathcal{O}_{Y}$-algebras

$$
\beta^{(m)}: \mathcal{A}^{(m)}\left(f_{1}, \ldots, f_{r}\right) \rightarrow \mathcal{P}\left(\mathcal{I}^{(m-1)}\right)
$$

sending $T_{i}$ to $(p-1) !\left(f_{i}^{p^{m-1}}\right)^{[p]}$ for $i=1, \ldots, r$. Let us also denote by $\rho^{(m)}$ the canonical PD-morphism $\mathcal{P}\left(\mathcal{I}^{(m)}\right) \rightarrow \mathcal{P}\left(\mathcal{I}^{(m-1)}\right)$. For all $i$ and all $k \geq 0$, we have

$$
\begin{aligned}
\beta^{(m)} \circ \alpha^{(m)}\left(\left(f_{i}^{p^{m}}\right)^{[k]}\right) & =\beta^{(m)}\left(p^{[k]} T_{i}^{k}\right) \\
& =p^{[k]}\left((p-1) !\left(f_{i}^{p^{m-1}}\right)^{[p]}\right)^{k} \\
& =\left(p(p-1) !\left(f_{i}^{p^{m-1}}\right)^{[p]}\right)^{[k]} \\
& =\left(\rho^{(m)}\left(f_{i}^{p^{m}}\right)\right)^{[k]}=\rho^{(m)}\left(\left(f_{i}^{p^{m}}\right)^{[k]}\right) .
\end{aligned}
$$

As the products $\left(f_{1}^{p^{m}}\right)^{\left[k_{1}\right]} \cdots\left(f_{r}^{p^{m}}\right)^{\left[k_{r}\right]}$ generate $\mathcal{P}\left(\mathcal{I}^{(m)}\right)$ as an $\mathcal{O}_{Y}$-module, we get that

$$
\beta^{(m)} \circ \alpha^{(m)}=\rho^{(m)} .
$$

Recall that, $\mathbb{Y}$ being noetherian and separated, the functors $H^{q}(\mathbb{U},-)$ commute with direct limits for any open subset $U \subset Y$ and any $q \geq 0$. In particular, we have

$$
\Gamma(\mathbb{U}, \mathcal{R})=\Gamma\left(\mathbb{U}, \mathcal{O}_{Y}\right)\left[T_{1}, \ldots, T_{r}\right]
$$

for any open subset $U \subset \mathbb{Y}$, and, by [3, Prop. 3.1.1], $\mathcal{R}$ is a coherent sheaf of rings on Y. As $\mathcal{A}^{(m)}\left(f_{1}, \ldots, f_{r}\right)$ is finitely presented over $\mathcal{R}$, it is coherent too, and the same holds for the kernel $\mathcal{K}_{n}$ of multiplication by $p^{n}$ on $\mathcal{A}^{(m)}\left(f_{1}, \ldots, f_{r}\right)$ for any $n$. Therefore, $\mathcal{K}_{n}$ is a coherent $\mathcal{O}_{Y_{n}}\left[T_{1}, \ldots, T_{r}\right]$ module, which implies that it is generated by its global sections, and so is the subsheaf $\mathcal{K}=\cup_{n} \mathcal{K}_{n}$ of $p$-torsion sections of $\mathcal{A}^{(m)}\left(f_{1}, \ldots, f_{r}\right)$.

On the other hand, the ideal $\mathcal{N}=\sum_{i} \mathcal{R} \cdot f_{i}$ is the increasing union of the $\mathcal{O}_{Y}$-coherent subsheaves $\mathcal{N}_{d}=\sum_{i} \mathcal{R}_{d} \cdot f_{i}$, where $\mathcal{R}_{d} \subset \mathcal{R}$ is the subsheaf of sections of total degree $\leq d$. Therefore, $H^{1}(\mathbb{Y}, \mathcal{N})=0$, and 
$\Gamma\left(\mathbb{Y}, \mathcal{A}^{(m)}\left(f_{1}, \ldots, f_{r}\right)\right)$ is a quotient of $\Gamma(\mathbb{Y}, \mathcal{R})$, hence is a noetherian ring. So there is an integer $s \geq 0$ such that $p^{s} g=0$ for any $p$-torsion section $g \in \Gamma\left(Y, \mathcal{A}^{(m)}\left(f_{1}, \ldots, f_{r}\right)\right)$. Since $\mathcal{K}$ is generated by its global sections on Y, we obtain that $p^{s} \mathcal{K}=0$.

The homomorphism $\alpha^{(m)}$ maps the torsion ideal $\mathcal{T}\left(\mathcal{I}^{(m)}\right) \subset \mathcal{P}\left(\mathcal{I}^{(m)}\right)$ to $\mathcal{K}$. Using (5.4.4), it follows that $p^{s} \rho^{(m)}$ vanishes on $\mathcal{T}\left(\mathcal{I}^{(m)}\right)$, which provides a factorization of $p^{s} \rho^{(m)}$ through $\overline{\mathcal{P}}\left(\mathcal{I}^{(m)}\right)$. Reducing $\bmod p^{n}$ for all $n$ and taking the associated morphism of crystals, we get a morphism $\sigma_{s}^{(m)}: \overline{\mathcal{P}}_{X, Y}^{(m)} \rightarrow \mathcal{P}_{X, Y}^{(m-1)}$, which satisfies the first relation of (5.4.1). As $\pi^{(m)}$ is an epimorphism, the second one follows.

5.5. We can now apply the constructions of 5.1 to the crystals $\overline{\mathcal{P}}_{X, Y}^{(m)}$. Taking their evaluations on the thickenings $Y \hookrightarrow W_{n} Y$, we obtain again an inverse system of $W_{n} \mathcal{O}_{Y}$-modules $\left(\overline{\mathcal{P}}_{X, Y, n}^{(m) W}\right)_{m, n}$ and an inverse system of complexes $\left(\overline{\mathcal{P}}_{X, Y, n}^{(m) W} \otimes W_{n} \mathcal{O}_{Y} W_{n} \Omega_{Y}^{\bullet}\right)_{m, n}$. We now define

$$
\begin{aligned}
& \widetilde{\mathcal{P}}_{X, Y}^{(m) W}:=\lim _{\overleftarrow{n}} \overline{\mathcal{P}}_{X, Y, n}^{(m) W}, \quad \widetilde{\mathcal{A}}_{X, Y}^{W}:=\lim _{\overleftarrow{m}}\left(\widetilde{\mathcal{P}}_{X, Y}^{(m) W} \otimes K\right), \\
& \widetilde{\mathcal{P}}_{X, Y}^{(m) W} \widehat{\otimes}_{W \mathcal{O}_{Y}} W \Omega_{Y}^{\bullet}:=\lim _{\overleftarrow{n}}\left(\overline{\mathcal{P}}_{X, Y, n}^{(m) W} \otimes_{W_{n} \mathcal{O}_{Y}} W_{n} \Omega_{Y}^{\bullet}\right), \\
& \left.\widetilde{\mathcal{A}}_{X, Y}^{W} \widehat{\otimes}_{W \mathcal{O}_{Y}} W \Omega_{Y}^{\bullet}:=\lim _{\overleftarrow{m}}\left(\widetilde{\mathcal{P}}_{X, Y}^{(m) W} \widehat{\otimes}_{W \mathcal{O}_{Y}} W \Omega_{Y}^{\bullet}\right) \otimes K\right)
\end{aligned}
$$

In degree 0 , these complexes are respectively equal to $\widetilde{\mathcal{P}}_{X, Y}^{(m) W}$ and $\widetilde{\mathcal{A}}_{X, Y}^{W}$. We will denote by $\tilde{\rho}^{(m)}: \widetilde{\mathcal{P}}_{X, Y}^{(m) W} \rightarrow \widetilde{\mathcal{P}}_{X, Y}^{(m-1) W}$ and $\tilde{\rho}^{(m) \bullet}: \widetilde{\mathcal{P}}_{X, Y}^{(m) W} \widehat{\otimes}_{W \mathcal{O}_{Y}} W \Omega_{Y}^{\bullet} \rightarrow$ $\widetilde{\mathcal{P}}_{X, Y}^{(m-1) W} \widehat{\otimes}_{W \mathcal{O}_{Y}} W \Omega_{Y}^{\bullet}$ the transition morphisms.

By functoriality, the morphisms of crystals $\pi^{(m)}: \mathcal{P}_{X, Y}^{(m)} \rightarrow \overline{\mathcal{P}}_{X, Y}^{(m)}$ define homomorphisms of $W \mathcal{O}_{Y}$-algebras

$$
\hat{\pi}^{(m)}: \widehat{\mathcal{P}}_{X, Y}^{(m) W} \rightarrow \widetilde{\mathcal{P}}_{X, Y}^{(m) W}, \quad \pi: \mathcal{A}_{X, Y}^{W} \rightarrow \widetilde{\mathcal{A}}_{X, Y}^{W},
$$

and morphisms of complexes on $Y$ (supported in $X$ )

$$
\begin{gathered}
\hat{\pi}^{(m) \bullet}: \widehat{\mathcal{P}}_{X, Y}^{(m) W} \widehat{\otimes}_{W \mathcal{O}_{Y}} W \Omega_{Y}^{\bullet} \rightarrow \widetilde{\mathcal{P}}_{X, Y}^{(m) W} \widehat{\otimes}_{W \mathcal{O}_{Y}} W \Omega_{Y}^{\bullet} \\
\pi^{\bullet}: \mathcal{A}_{X, Y}^{W} \widehat{\otimes}_{W \mathcal{O}_{Y}} W \Omega_{Y}^{\bullet} \rightarrow \widetilde{\mathcal{A}}_{X, Y}^{W} \widehat{\otimes}_{W \mathcal{O}_{Y}} W \Omega_{Y}^{\bullet} .
\end{gathered}
$$

Proposition 5.6. Let $Y$ be a smooth $k$-scheme, and let $X \subset Y$ be a closed subscheme. 
(i) The morphism $\pi^{\bullet}$ defined by (5.5.3) is an isomorphism.

(ii) For all $m \geq 0, j \geq 0$ and $i \geq 1$, we have

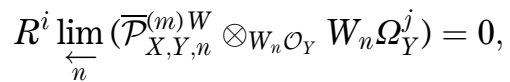

$$
\begin{aligned}
& R^{i}{\underset{\leftarrow}{m}}_{\lim _{X, Y}}\left(\left(\widetilde{\mathcal{P}}_{X, Y}^{(m) W} \widehat{\otimes}_{W \mathcal{O}_{Y}} W \Omega_{Y}^{j}\right) \otimes K\right)=0 .
\end{aligned}
$$

In particular, the homomorphism $\pi$ of (5.5.1) is an isomorphism.

Proof. Applying Lemma 5.4, we can find an increasing sequence of integers $(s(m))_{m \geq 1}$ such that, for each $m$, there exists a morphism of crystals $\sigma_{s(m)}^{(m)}: \overline{\mathcal{P}}_{X, Y}^{(m)} \rightarrow \mathcal{P}_{X, Y}^{(m-1)}$ satisfying (5.4.1) for $s=s(m)$. For any $m \geq 1$, these morphisms satisfy the relation

$$
p^{s(m+1)-s(m)} \sigma_{s(m)}^{(m)} \circ \bar{\rho}^{(m+1)}=\rho^{(m)} \circ \sigma_{s(m+1)}^{(m+1)},
$$

which follows from (5.4.1) because $\pi^{(m+1)}$ is an epimorphism.

By functoriality, the morphisms $\sigma_{s(m)}^{(m)}$ define homomorphisms of $W \mathcal{O}_{Y^{-}}$ algebras $\tilde{\sigma}_{s(m)}^{(m)}: \widetilde{\mathcal{P}}_{X, Y}^{(m) W} \rightarrow \widehat{\mathcal{P}}_{X, Y}^{(m-1) W}$ and morphisms of complexes

$$
\tilde{\sigma}_{s(m)}^{(m)}: \widetilde{\mathcal{P}}_{X, Y}^{(m) W} \widehat{\otimes}_{W \mathcal{O}_{Y}} W \Omega_{Y}^{\bullet} \rightarrow \widehat{\mathcal{P}}_{X, Y}^{(m-1) W} \widehat{\otimes}_{W \mathcal{O}_{Y}} W \Omega_{Y}^{\bullet}
$$

They satisfy the analogs of relations (5.4.1) and (5.6.3). If we set

$$
\psi^{(m) \bullet}=p^{-s(m)} \tilde{\sigma}_{s(m)}^{(m)}: \widetilde{\mathcal{P}}_{X, Y}^{(m) W} \widehat{\otimes}_{W \mathcal{O}_{Y}} W \Omega_{Y}^{\bullet} \otimes K \rightarrow \widehat{\mathcal{P}}_{X, Y}^{(m-1) W} \widehat{\otimes}_{W \mathcal{O}_{Y}} W \Omega_{Y}^{\bullet} \otimes K,
$$

we obtain when $m$ varies a family of morphisms of complexes, which commute with the transition morphisms $\hat{\rho}^{(m) \bullet}$ and $\tilde{\rho}^{(m) \bullet}$ and satisfy

$$
\psi^{(m) \bullet} \circ \hat{\pi}^{(m) \bullet}=\hat{\rho}^{(m) \bullet}, \quad \hat{\pi}^{(m-1) \bullet} \circ \psi^{(m) \bullet}=\tilde{\rho}^{(m) \bullet} .
$$

Taking the inverse limit of the morphisms $\psi^{(m)} \bullet$ when $m$ varies provides a morphism of complexes

$$
\psi^{\bullet}: \widetilde{\mathcal{A}}_{X, Y}^{W} \widehat{\otimes}_{W \mathcal{O}_{Y}} W \Omega_{Y}^{\bullet} \rightarrow \mathcal{A}_{X, Y}^{W} \widehat{\otimes}_{W \mathcal{O}_{Y}} W \Omega_{Y}^{\bullet},
$$

which is inverse to $\pi^{\bullet}$.

To prove assertion (ii), we observe first that, by 2.12 and Lemma 4.3, $\overline{\mathcal{P}}_{X, Y, n}^{(m) W} \otimes_{W_{n} \mathcal{O}_{Y}} W_{n} \Omega_{Y}^{j}$ is a quasi-coherent $W_{n} \mathcal{O}_{Y}$-module, for all $n \geq 1, m \geq 0$. So, for fixed $m$ and variable $n$, the inverse system $\left(\overline{\mathcal{P}}_{X, Y, n}^{(m) W} \otimes_{W_{n} \mathcal{O}_{Y}} W_{n} \Omega_{Y}^{j}\right)_{n \geq 1}$ has surjective transition morphisms and terms with vanishing cohomology in degree $\geq 1$ on any affine open subset of $Y$. Therefore (5.6.1) follows from the algebraic Mittag-Leffler criterion [12, Prop. (13.2.3)]. 
Note that this Mittag-Leffler criterion also implies that, for any affine open subset $U \subset Y$, any $i \geq 1$, and any $j$,

$$
H^{i}\left(U, \widetilde{\mathcal{P}}_{X, Y}^{(m) W} \widehat{\otimes}_{W \mathcal{O}_{Y}} W \Omega_{Y}^{j}\right)=0 .
$$

Since $U$ is quasi-compact and separated, it follows that, for $i \geq 1$,

$$
H^{i}\left(U,\left(\widetilde{\mathcal{P}}_{X, Y}^{(m) W} \widehat{\otimes}_{W \mathcal{O}_{Y}} W \Omega_{Y}^{j}\right) \otimes K\right)=0 .
$$

So, in order to prove (5.6.2), it suffices to prove that each $K$-vector space $\Gamma\left(U,\left(\widetilde{\mathcal{P}}_{X, Y}^{(m) W} \widehat{\otimes}_{W \mathcal{O}_{Y}} W \Omega_{Y}^{j}\right) \otimes K\right)$ can be endowed with a metrizable topological vector space structure for which it is separated and complete, and such that the transition maps for varying $m$ are continuous and satisfy the topological Mittag-Leffler condition (ML') [12, $\mathrm{O}_{\mathrm{III}}$, Rem. (13.2.4) (i)]. Since, by construction, $\widetilde{\mathcal{P}}_{X, Y}^{(m) W}$ is flat over $W$, Proposition 2.13 allows to use for this purpose the $p$-adic topology, defined by the lattice $\Gamma\left(U, \widetilde{\mathcal{P}}_{X, Y}^{(m) W} \widehat{\otimes}_{W \mathcal{O}_{Y}} W \Omega_{Y}^{j}\right)$, which is metrizable, separated, and complete. To finish the proof, it is then sufficient to prove that, for all $m \geq 0$, the image of $\Gamma\left(U, W \Omega_{Y}^{j} \otimes K\right)$ in $\Gamma\left(U,\left(\widetilde{\mathcal{P}}_{X, Y}^{(m) W} \widehat{\otimes}_{W \mathcal{O}_{Y}} W \Omega_{Y}^{j}\right) \otimes K\right)$ is dense for the $p$ adic topology in the image of $\Gamma\left(U,\left(\widetilde{\mathcal{P}}_{X, Y}^{(m+1) W} \widehat{\otimes}_{W \mathcal{O}_{Y}} W \Omega_{Y}^{j}\right) \otimes K\right)$. As multiplication by $p$ is a homeomorphism of $\Gamma\left(U,\left(\widetilde{\mathcal{P}}_{X, Y}^{(m) W} \widehat{\otimes}_{W \mathcal{O}_{Y}} W \Omega_{Y}^{j}\right) \otimes K\right)$ for the $p$-adic topology, it is enough to show that, for any $n \geq 0$,

$$
\begin{aligned}
& \tilde{\rho}^{(m+1)}\left(\Gamma\left(U, \widetilde{\mathcal{P}}_{X, Y}^{(m+1) W} \widehat{\otimes}_{W \mathcal{O}_{Y}} W \Omega_{Y}^{j}\right)\right) \subset \\
& \Gamma\left(U, W \Omega_{Y}^{j}\right) \otimes K+p^{n} \Gamma\left(U, \widetilde{\mathcal{P}}_{X, Y}^{(m) W} \widehat{\otimes}_{W \mathcal{O}_{Y}} W \Omega_{Y}^{j}\right) .
\end{aligned}
$$

Let $f_{1}, \ldots, f_{r} \in \Gamma\left(U, \mathcal{O}_{Y}\right)$ be a family of generators over $U$ of the ideal $\mathcal{I}_{Y}$ of $X$ in $Y$. Then, for all $i \geq 2$ and all $m \geq 0$, the kernel $\mathcal{K}_{i}^{(m)}$ of the homomorphism $W_{i} \mathcal{O}_{Y} \rightarrow \mathcal{O}_{X^{(m)}}$ is generated by $V W_{i-1} \mathcal{O}_{Y}$ and by the Teichmüller representatives $\left[f_{1}^{p^{m}}\right], \ldots,\left[f_{r}^{p^{m}}\right]$. By construction, $\overline{\mathcal{P}}_{X, Y, i}^{(m) W}$ is a quotient of the divided power envelope of $\mathcal{K}_{i}^{(m)}$ with compatibility with the canonical divided powers of $V W_{i-1} \mathcal{O}_{Y}$. This implies that $\overline{\mathcal{P}}_{X, Y, i}^{(m) W}$ is generated as a $W_{i} \mathcal{O}_{Y}$-module by the sections

$$
\left.\left([]_{-}\right]^{p^{m}}\right)^{[\underline{k}]}:=\left(\left[f_{1}\right]^{p^{m}}\right)^{\left[k_{1}\right]} \cdots\left(\left[f_{r}\right]^{p^{m}}\right)^{\left[k_{r}\right]},
$$

for $\underline{k}=\left(k_{1}, \ldots, k_{r}\right) \in \mathbb{N}^{r}$. Therefore, an element $x \in \Gamma\left(U, \widetilde{\mathcal{P}}_{X, Y}^{(m+1) W} \widehat{\otimes}_{W \mathcal{O}_{Y}} W \Omega_{Y}^{j}\right)$ can be written as an infinite sum

$$
x=\sum_{\underline{k}}\left([f]^{p^{m+1}}\right)^{[\underline{k}]} \otimes \omega_{\underline{k}},
$$

where the sections $\omega_{\underline{k}} \in \Gamma\left(U, W \Omega_{Y}^{j}\right)$ are such that, for any $i$, $\omega_{\underline{k}} \in \Gamma\left(U, \mathrm{Fil}^{i} W \Omega_{Y}^{j}\right)$ for all but a finite number of $\underline{k}$ 's. As $\tilde{\rho}^{(m+1)}$ is a PD- 
morphism, we can write

$$
\left.\tilde{\rho}^{(m+1)}(x)=\sum_{\underline{k}}\left(\left([f]^{p^{m}}\right)^{p}\right)^{[\underline{k}]} \otimes \omega_{\underline{k}}=\sum_{\underline{k}} \frac{(p \underline{k}) !}{\underline{k} !}([f]]^{p^{m}}\right)^{[p \underline{k}]} \otimes \omega_{\underline{k}} .
$$

Using the classical formula for the $p$-adic valuation of $v_{p}(k !)$ [7, Lemma 3.3], we get

$$
v_{p}\left(\frac{(p k) !}{k !}\right)=k
$$

So we can write

$$
\left.\left.\tilde{\rho}^{(m+1)}(x)=\sum_{\forall j, k_{j}<n} \frac{(p \underline{k}) !}{\underline{k} !}\left([]_{-}\right]^{p^{m}}\right)^{[p \underline{k}]} \otimes \omega_{\underline{k}}+p^{n} \sum_{\exists j, k_{j} \geq n} \frac{(p \underline{k}) !}{\underline{k} ! p^{n}}\left([]_{-}\right]^{p^{m}}\right)^{[p \underline{p}]} \otimes \omega_{\underline{k}},
$$

a decomposition in which the first term belongs to $\Gamma\left(U, W \Omega_{Y}^{j}\right) \otimes K$ and the second one to $p^{n} \Gamma\left(U, \widetilde{\mathcal{P}}_{X, Y}^{(m) W} \widehat{\otimes}_{W \mathcal{O}_{Y}} W \Omega_{Y}^{j}\right)$. This proves (5.6.5), and ends the proof of the proposition.

5.7. Proof of Theorem 5.2. Let us assume first that $Y$ can be embedded as a closed subscheme in a smooth formal scheme $\mathbb{P}$ endowed with a lifting $F$ of the absolute Frobenius endomorphism. For $n \geq 1$, let $P_{n}$ be the reduction of $\mathbb{P}$ over $W_{n}$. We denote by $\mathcal{J}$ the ideal of $X$ in $\mathbb{P}, \mathcal{J}_{n}$ its ideal in $P_{n}$, and we keep the notation of 4.4 and 5.1. Let $\mathcal{K}$ be the ideal of $Y$ in $\mathbb{P}$, and $\mathcal{K}_{n}$ its ideal in $P_{n}$.

Let $\mathcal{P}(\mathcal{K}), \mathcal{P}\left(\mathcal{K}_{n}\right) \simeq \mathcal{P}(\mathcal{K}) / p^{n} \mathcal{P}(\mathcal{K})$ be the divided powers envelopes of $\mathcal{K}$ and $\mathcal{K}_{n}$. We denote by $T_{n}$ the PD-thickening of $Y$ given by $T_{n}=\operatorname{Spec} \mathcal{P}\left(\mathcal{K}_{n}\right)$ and by $\mathcal{P}_{X, Y, n}^{(m) T}, \overline{\mathcal{P}}_{X, Y, n}^{(m) T}$ the evaluations on $T_{n}$ of the crystals $\mathcal{P}_{X, Y}^{(m)}, \overline{\mathcal{P}}_{X, Y}^{(m)}$. Let $\mathcal{K}_{T_{n}}=\operatorname{Ker}\left(\mathcal{O}_{T_{n}} \rightarrow \mathcal{O}_{Y}\right)$ be the PD-ideal generated by $\mathcal{K}_{n}$ in $\mathcal{P}\left(\mathcal{K}_{n}\right)$, and let $\delta$ be its canonical PD-structure. Then the ideal $\mathcal{I}_{T_{n}}$ of $X$ in $T_{n}$ is given by

$$
\mathcal{I}_{T_{n}}=\mathcal{J}_{n} \mathcal{O}_{T_{n}}+\mathcal{K}_{T_{n}} .
$$

Therefore,

$$
\left(\mathcal{I}_{T_{n}}\right)_{\mathcal{K}_{T_{n}}}^{(m)}=\mathcal{J}_{n}^{(m)} \mathcal{O}_{T_{n}}+\mathcal{K}_{T_{n}},
$$

and the description (5.1.2) of $\mathcal{P}_{X, Y, n}^{(m) T}$ shows that

$$
\mathcal{P}_{X, Y, n}^{(m) T}=\mathcal{P}_{\delta}\left(\mathcal{J}_{n}^{(m)} \mathcal{O}_{T_{n}}+\mathcal{K}_{T_{n}}\right)=\mathcal{P}_{\delta}\left(\mathcal{J}_{n}^{(m)} \mathcal{O}_{T_{n}}\right) .
$$

But the universal property of divided power envelopes provides formally a canonical isomorphism

$$
\mathcal{P}_{\delta}\left(\mathcal{J}_{n}^{(m)} \mathcal{O}_{T_{n}}+\mathcal{K}_{T_{n}}\right) \simeq \mathcal{P}\left(\mathcal{J}_{n}^{(m)}+\mathcal{K}_{n}\right) .
$$


So we finally get a canonical isomorphism

$$
\mathcal{P}_{X, Y, n}^{(m) T} \simeq \mathcal{P}\left(\mathcal{J}_{n}^{(m)}+\mathcal{K}_{n}\right) .
$$

Let

$$
\begin{aligned}
& \widehat{\mathcal{P}}_{X, Y}^{(m) T}=\lim _{\overleftarrow{n}} \mathcal{P}_{X, Y, n}^{(m) T}=\widehat{\mathcal{P}}\left(\mathcal{J}^{(m)}+\mathcal{K}\right), \\
& \widetilde{\mathcal{P}}_{X, Y}^{(m) T}=\lim _{\overleftarrow{n}} \overline{\mathcal{P}}_{X, Y, n}^{(m) T}=\widetilde{\mathcal{P}}\left(\mathcal{J}^{(m)}+\mathcal{K}\right) .
\end{aligned}
$$

We get by (3.5.3) canonical morphisms of complexes that sit in a commutative square

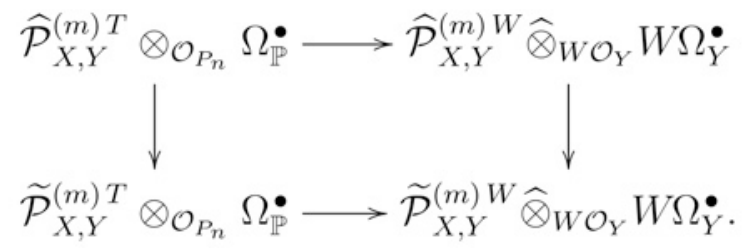

Tensorizing by $K$ and taking the inverse limit for variable $m$, we get a similar square

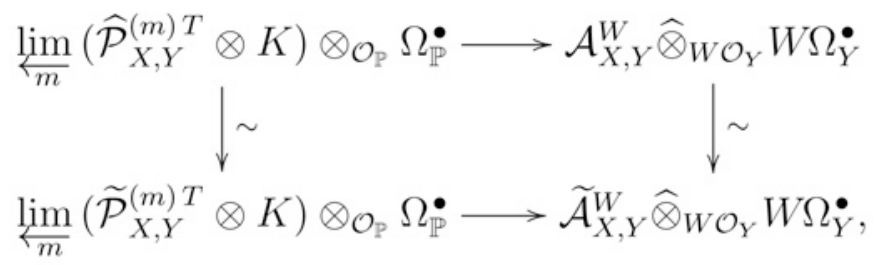

in which the right vertical arrow is an isomorphism thanks to Proposition 5.6. The left vertical one is also an isomorphism, because Lemma 5.4 implies by the same argument that the homomorphism

$$
\lim _{\overleftarrow{m}}\left(\widehat{\mathcal{P}}_{X, Y}^{(m) T} \otimes K\right) \rightarrow \lim _{\overleftarrow{m}}\left(\widetilde{\mathcal{P}}_{X, Y}^{(m) T} \otimes K\right)
$$

which is induced by the morphism of inverse systems of crystals $\pi^{(m)}: \mathcal{P}_{X, Y}^{(m)} \rightarrow \overline{\mathcal{P}}_{X, Y}^{(m)}$, is an isomorphism. On the other hand, Corollary 3.8 implies that the lower horizontal arrow of (5.7.2) is a quasi-isomorphism, since the crystal $\overline{\mathcal{P}}_{X, Y}^{(m)}$ is flat over $W$ and quasi-coherent. For all $j$, the inverse system $\left(\widetilde{\mathcal{P}}_{X, Y}^{(m) T} \otimes K \otimes \Omega_{\mathrm{P}}^{j}\right)_{m \geq 0}$ is lim-acyclic by (5.7.1) and (4.5.2), and the inverse system $\left(\left(\widetilde{\mathcal{P}}_{X, Y}^{(m) W} \widehat{\otimes}_{W \mathcal{O}_{Y}} W \Omega_{Y}^{\overleftarrow{j}}\right) \otimes K\right)_{m \geq 0}$ is lim-acyclic by (5.6.2). Therefore, the lower horizontal arrow of (5.7.3) is again a quasi-isomorphism, and the upper horizontal arrow is a quasi-isomorphism too. 
We now consider the canonical morphism of complexes defined by the commutative diagram

$$
\begin{aligned}
& \mathcal{A}_{X, \mathbb{P}} \otimes_{\mathcal{O}_{\mathbb{P}}} \Omega_{\mathbb{P}}^{\bullet} \lim _{m}\left(\widehat{\mathcal{P}}_{X, Y}^{(m) T} \otimes K\right) \otimes_{\mathcal{O}_{\mathbb{P}}} \Omega_{\mathbb{P}}^{\bullet} \\
& \text { (4.5.1) } \downarrow \sim \\
& \lim _{m} \widehat{\mathcal{P}}\left(\mathcal{J}^{(m)}\right)_{K} \otimes_{\mathcal{O}_{\mathbb{P}}} \Omega_{\mathbb{P}}^{\bullet} \lim _{\leftarrow_{m}} \widehat{\mathcal{P}}\left(\mathcal{J}^{(m)}+\mathcal{K}\right)_{K} \otimes_{\mathcal{O}_{\mathbb{P}}} \Omega_{\mathbb{P}}^{\bullet} .
\end{aligned}
$$

In this diagram, the lower horizontal arrow is the arrow (4.7.4) induced by the functoriality map for PD-envelopes. As shown in the proof of Theorem 4.7, this arrow is a quasi-isomorphism. Therefore, we can define the quasi-isomorphism (5.2.1) to be the composition of the morphism (5.7.4) with the upper horizontal arrow of diagram (5.7.3). Each of these two morphisms is functorial in an obvious way with respect to the data $(X, Y, \mathbb{P}, F)$, and, as isomorphisms

$$
\begin{gathered}
\mathbb{R} \underline{\Gamma}_{\text {rig }}(X / K) \stackrel{\sim}{\longrightarrow} \mathbb{R} \lim _{\overleftarrow{m}}\left(\mathbb{R} u_{Y / W *} \mathcal{P}_{X, Y}^{(m)}\right)_{K}, \\
\mathbb{R} \lim _{\overleftarrow{m}}\left(\mathbb{R} u_{Y / W *} \mathcal{P}_{X, Y}^{(m)}\right)_{K} \stackrel{\sim}{\longrightarrow} \mathcal{A}_{X, Y}^{W} \widehat{\otimes}_{W \mathcal{O}_{Y}} W \Omega_{Y}^{\bullet}
\end{gathered}
$$

in the derived category $D^{\mathrm{b}}(X, K)$, they are independent of the choice of the embedding into $(\mathbb{P}, F)$ (by the usual diagonal embedding argument). This proves assertion (i) of Theorem 5.2.

To prove assertion (ii), we argue again as in 3.3. We choose an affine open covering $\mathfrak{U}=\left(U_{\alpha}\right)$ of $Y$, and, for each $\alpha$, we choose a closed immersion $U_{\alpha} \hookrightarrow \mathbb{P}_{\alpha}$ into a smooth formal scheme endowed with a lifting $F_{\alpha}$ of the absolute Frobenius endomorphism. For each multi-index $\underline{\alpha}=\left(\alpha_{0}, \ldots, \alpha_{i}\right)$, we endow the product $\mathbb{P}_{\underline{\alpha}}=P_{\alpha_{0}} \times_{W} \cdots \times_{W} P_{\alpha_{i}}$ with the endomorphism $F_{\underline{\alpha}}=F_{\alpha_{0}} \times \cdots \times F_{\alpha_{i}}$, and we embedd $U_{\underline{\alpha}}=U_{\alpha_{0}} \cap \ldots \cap U_{\alpha_{i}}$ diagonally into $\mathbb{P}_{\underline{\alpha}}$. Let $j_{\underline{\alpha}}$ be the inclusion of $X \cap U_{\underline{\alpha}}$ into $X$. As a representative of $\mathbb{R} \underline{\Gamma}_{\text {rig }}(X / \bar{K})$, we use the total complex associated to the Cech bicomplex described in 4.1: in bidegree $(j, i)$, it is given by

$$
\prod_{\underline{\alpha}=\left(\alpha_{0}, \ldots, \alpha_{i}\right)} j_{\underline{\alpha} *}\left(\mathcal{A}_{X \cap U_{\underline{\alpha}}, \mathbb{P}_{\underline{\alpha}}} \otimes \Omega_{\mathbb{P}_{\underline{\underline{\alpha}}}}^{j}\right) .
$$

On the other hand, we can represent $\mathcal{A}_{X, Y}^{W} \widehat{\otimes}_{W \mathcal{O}_{Y}} W \Omega_{Y}^{\bullet}$ by its Čech resolution defined by the covering $\mathfrak{l}$, which is the total complex associated to the bicomplex defined in bidegree $(j, i)$ by

$$
\prod_{\underline{\alpha}=\left(\alpha_{0}, \ldots, \alpha_{i}\right)} j_{\underline{\alpha} *}\left(\mathcal{A}_{X \cap U_{\underline{\alpha}}, U_{\underline{\alpha}}}^{W} \widehat{\otimes}_{W \mathcal{O}_{U_{\underline{\alpha}}}} W \Omega_{U_{\underline{\alpha}}}^{j}\right) .
$$


For fixed $\underline{\alpha}$ and variable $j$, we have a quasi-isomorphism of complexes (5.2.1) from $\mathcal{A}_{X \cap U_{\underline{\alpha}}, \mathrm{P}_{\underline{\alpha}}} \otimes \Omega_{\mathrm{P}_{\underline{\alpha}}}^{j}$ to $\mathcal{A}_{X \cap U_{\underline{\alpha}}, U_{\underline{\alpha}}}^{W} \widehat{\otimes}_{W \mathcal{O}_{U_{\underline{\alpha}}}} W \Omega_{U_{\alpha}}^{j}$, and its direct image by $j_{\underline{\alpha}}$ remains a quasi-isomorphism, because $j_{\underline{\alpha}}$ is an affine morphism, and, by Mittag-Leffler, the terms of these complexes have no higher cohomology on affine subsets. The functoriality of (5.2.1) implies that these quasi-isomorphisms commute with the differential of the Čech complexes when $\underline{\alpha}$ varies. Therefore they induce a quasi-isomorphism between the associated total complexes, and (5.2.2) is defined by its image in $D^{\mathrm{b}}(X, K)$.

To check that (5.2.2) does not depend on the choices, one considers another covering $\mathfrak{U}^{\prime}=\left(U_{\alpha^{\prime}}^{\prime}\right)$, with closed immersions $U_{\alpha^{\prime}}^{\prime} \hookrightarrow\left(\mathbb{P}_{\alpha^{\prime}}^{\prime}, F_{\alpha^{\prime}}^{\prime}\right)$. One can then refine both $\mathfrak{U}$ and $\mathfrak{U}^{\prime}$ by the covering by the intersections $U_{\alpha} \cap U_{\alpha^{\prime}}^{\prime}$, which can be embedded into $\left(\mathbb{P}_{\alpha} \times{ }_{W} \mathbb{P}_{\alpha^{\prime}}^{\prime}, F_{\alpha} \times F_{\alpha^{\prime}}^{\prime}\right)$. Then the functoriality of (5.2.1) provides commutative diagrams showing that the morphisms (5.2.2) constructed by means of $\mathfrak{U}$ and $\mathfrak{U}^{\prime}$ are equal in $D^{\mathrm{b}}(X, K)$. For a morphism of couples $(w, v):\left(X^{\prime}, Y^{\prime}\right) \rightarrow(X, Y)$, a similar argument using affine coverings $\mathfrak{U}^{\prime}, \mathfrak{U}$ of $Y^{\prime}, Y$, and the embeddings $U_{\alpha^{\prime}}^{\prime} \cap v^{-1}\left(U_{\alpha}\right) \hookrightarrow \mathbb{P}_{\alpha^{\prime}}^{\prime} \times \mathbb{P}_{\alpha}$ defined by the graph of $v$, shows that the morphisms (5.2.2) for $\left(X^{\prime}, Y^{\prime}\right)$ and $(X, Y)$ commute with the functoriality morphisms on the sources and targets. This completes the proof of assertion (ii).

Finally, in view of the definition of rigid cohomology for proper schemes recalled in 4.1, assertion (iii) follows from assertion (ii) by taking sections on $X$.

5.8. We now briefly explain how to extend the previous results to rigid cohomology with compact supports.

Let $X$ be a $k$-scheme of finite type, $Z \subset X$ a closed subscheme, and $U=X \backslash Z$. We generalize the complexes entering in Theorem 5.2 as follows.

a) Assume that there exists a closed immersion $X \hookrightarrow \mathbb{P}$ of $X$ into a smooth formal scheme $\mathbb{P}$. By functoriality, we obtain a canonical morphism of complexes

$$
\mathcal{A}_{X, \mathrm{P}} \otimes_{\mathcal{O}_{\mathrm{P}}} \Omega_{\mathrm{P}}^{\bullet} \rightarrow \mathcal{A}_{Z, \mathrm{P}} \otimes_{\mathcal{O}_{\mathrm{P}}} \Omega_{\mathrm{P}}^{\bullet}
$$

which we view as bicomplex. We will use the shorter notation

$$
\mathcal{A}_{X \backslash Z, \mathbb{P}}^{\mathrm{c}} \otimes_{\mathcal{O}_{\mathrm{P}}} \Omega_{\mathrm{P}}^{\bullet}:=\left(\mathcal{A}_{X, \mathbb{P}} \otimes_{\mathcal{O}_{\mathrm{P}}} \Omega_{\mathrm{P}}^{\bullet} \rightarrow \mathcal{A}_{Z, \mathbb{P}} \otimes_{\mathcal{O}_{\mathrm{P}}} \Omega_{\mathrm{P}}^{\bullet}\right)_{\mathrm{t}}
$$

for the associated total complex. From this definition, we obtain a short exact sequence of complexes

$$
0 \rightarrow \mathcal{A}_{Z, \mathrm{P}} \otimes_{\mathcal{O}_{\mathrm{P}}} \Omega_{\mathrm{P}}^{\bullet}[-1] \rightarrow \mathcal{A}_{X \backslash Z, \mathrm{P}}^{\mathrm{c}} \otimes_{\mathcal{O}_{\mathrm{P}}} \Omega_{\mathrm{P}}^{\bullet} \rightarrow \mathcal{A}_{X, \mathrm{P}} \otimes_{\mathcal{O}_{\mathrm{P}}} \Omega_{\mathrm{P}}^{\bullet} \rightarrow 0 .
$$


We will denote by $\mathbb{R} \underline{\Gamma}_{\text {rig,c }}(X \backslash Z / K)$ the image in $D^{\mathrm{b}}(X, K)$ of the complex $\mathcal{A}_{X \backslash Z, \mathrm{P}}^{\mathrm{c}} \otimes_{\mathcal{O}_{\mathrm{P}}} \Omega_{\mathrm{P}}^{\bullet}$; up to canonical isomorphism, it does not depend on the choice of the embedding of $X$ into $\mathbb{P}$.

b) Without embedding assumption, we can choose an affine covering $\mathfrak{U}=\left(U_{\alpha}\right)$ of $X$ and closed immersions $U_{\alpha} \hookrightarrow \mathbb{P}_{\alpha}$ into smooth formal schemes. We obtain a functoriality morphism between the Čech bicomplexes defined by (5.7.5) for $X$ and for $Z$, which we can view as a triple complex. We generalize the previous definition by denoting $\mathbb{R} \underline{\Gamma}_{\text {rig,c }}(X \backslash Z / K)$ the image in $D^{\mathrm{b}}(X, K)$ of the total complex associated to this triple complex. Up to canonical isomorphism, it is still independent of the choices. From the exact sequences (5.8.1) on intersections of open subsets of $\mathfrak{U}$, we get in $D^{\mathrm{b}}(X, K)$ an exact triangle

$$
\mathbb{R} \underline{\Gamma}_{\text {rig }, \mathrm{c}}(X \backslash Z / K) \rightarrow \mathbb{R} \underline{\Gamma}_{\text {rig }}(X / K) \rightarrow \mathbb{R} \underline{\Gamma}_{\text {rig }}(Z / K) \stackrel{+1}{\longrightarrow} .
$$

c) When $X$ is proper, the rigid cohomology of $U$ with compact support can be defined by

$$
\mathbb{R} \Gamma_{\text {rig }, \mathrm{c}}(U / K):=\mathbb{R} \Gamma\left(X, \mathbb{R} \underline{\Gamma}_{\text {rig }, \mathrm{c}}(X \backslash Z / K)\right) .
$$

Note that, under the assumptions of a), (4.1.2) provides a canonical isomorphism

$$
\mathbb{R} \Gamma_{\text {rig, }, \mathrm{c}}(U / K) \simeq \mathbb{R} \Gamma(] X\left[_{\mathrm{P}},\left(\Omega_{]_{X[\mathrm{P}}}^{\bullet} \rightarrow u_{*}\left(\Omega_{] Z\left[_{\mathrm{P}}\right.}^{\bullet}\right)\right)_{\mathrm{t}}\right),
$$

where $u$ denotes the inclusion $] Z[\mathrm{p} \hookrightarrow] X[\mathrm{p}$. Without embedding assumption, the complex $\mathbb{R} \Gamma_{\text {rig,c }}(U / K)$ does not depend on the compactification $X$ of $U$, and satisfies with respect to $U$ the usual functoriality properties of cohomology with compact supports (see [1, section 3] and $[19,6.4]$ for details). Taking sections on $X$, the triangle (5.8.2) yields the usual triangle

$$
\mathbb{R} \Gamma_{\text {rig }, \mathrm{c}}(U / K) \rightarrow \mathbb{R} \Gamma_{\text {rig }}(X / K) \rightarrow \mathbb{R} \Gamma_{\text {rig }}(Z / K) \stackrel{+1}{\longrightarrow} .
$$

d) Let now $i: X \hookrightarrow Y$ be a closed immersion of $X$ into a smooth $k$ scheme. By functoriality, we obtain a canonical morphism of complexes

$$
\mathcal{A}_{X, Y}^{W} \widehat{\otimes}_{W \mathcal{O}_{Y}} W \Omega_{Y}^{\bullet} \rightarrow \mathcal{A}_{Z, Y}^{W} \widehat{\otimes}_{W \mathcal{O}_{Y}} W \Omega_{Y}^{\bullet},
$$

which we consider again as a bicomplex. We will denote by

$$
\mathcal{A}_{X \backslash Z, Y}^{\mathrm{c}, W} \widehat{\otimes}_{W \mathcal{O}_{Y}} W \Omega_{Y}^{\bullet}:=\left(\mathcal{A}_{X, Y}^{W} \widehat{\otimes}_{W \mathcal{O}_{Y}} W \Omega_{Y}^{\bullet} \rightarrow \mathcal{A}_{Z, Y}^{W} \widehat{\otimes}_{W \mathcal{O}_{Y}} W \Omega_{Y}^{\bullet}\right)_{\mathrm{t}}
$$

the associated total complex, which sits in a short exact sequence of 
complexes

$$
\begin{aligned}
0 \rightarrow \mathcal{A}_{Z, Y}^{W} \widehat{\otimes}_{W \mathcal{O}_{Y}} W \Omega_{Y}^{\bullet}[-1] \rightarrow \mathcal{A}_{X \backslash Z, Y}^{\mathrm{c}, W} \widehat{\otimes}_{W \mathcal{O}_{Y}} W \Omega_{Y}^{\bullet} \rightarrow \\
\mathcal{A}_{X, Y}^{W} \widehat{\otimes}_{W \mathcal{O}_{Y}} W \Omega_{Y}^{\bullet} \rightarrow 0 .
\end{aligned}
$$

Corollary 5.9. Let $X$ be a k-scheme of finite type, $Z \subset X$ a closed subscheme, $U=X \backslash Z$, and $i: X \hookrightarrow Y$ a closed immersion in a smooth $k$ scheme.

(i) If there exists a closed immersion $Y \hookrightarrow \mathbb{P}$ of $Y$ into a smooth formal scheme $\mathbb{P}$ endowed with a Frobenius lifting, these data define a quasiisomorphism of complexes of sheaves of $K$-vector spaces supported in $X$

$$
\mathcal{A}_{X \backslash Z, \mathbb{P}}^{\mathrm{c}} \otimes_{\mathcal{O}_{\mathrm{P}}} \Omega_{\mathrm{P}}^{\bullet} \longrightarrow \mathcal{A}_{X \backslash Z, Y}^{\mathrm{c}, W} \widehat{\otimes}_{W \mathcal{O}_{Y}} W \Omega_{Y}^{\bullet},
$$

functorial with respect to $(Z, X, Y, \mathrm{P}, F)$. Its image in $D^{\mathrm{b}}(X, K)$ is independent of the choice of the embedding of $Y$ into $(\mathbb{P}, F)$. Together with the quasi-isomorphisms (5.2.1) for $X$ and $Z$, it defines a morphism of exact sequences from the exact sequence (5.8.1) to the exact sequence (5.8.4).

(ii) Without extra assumption, there exists in $D^{\mathrm{b}}(X, K)$ a canonical isomorphism

$$
\mathbb{R} \underline{\Gamma}_{\text {rig }, \mathrm{c}}(X \backslash Z / K) \stackrel{\sim}{\longrightarrow} \mathcal{A}_{X \backslash Z, Y}^{\mathrm{c}, W} \widehat{\otimes}_{W \mathcal{O}_{Y}} W \Omega_{Y}^{\bullet},
$$

functorial with respect to the triple $(Z, X, Y)$, which is defined by (5.9.1) whenever the assumptions of (i) are satisfied. Together with the isomorphisms (5.2.2) for $X$ and $Z$, it defines an isomorphism of exact triangles from (5.8.2) to the triangle defined by the exact sequence (5.8.4).

(iii) If $X$ is proper, there exists in $D^{\mathrm{b}}(K)$ a canonical isomorphism

$$
\mathbb{R} \Gamma_{\text {rig,c }}(U / K) \stackrel{\sim}{\longrightarrow} R\left(X, \mathcal{A}_{X \backslash Z, Y}^{\mathrm{c}, W} \widehat{\otimes}_{W \mathcal{O}_{Y}} W \Omega_{Y}^{\bullet}\right),
$$

functorial with respect to the triple $(Z, X, Y)$. Together with the isomorphisms (5.2.3) for $X$ and $Z$, it defines an isomorphism of exact triangles from (5.8.3) to the triangle obtained by applying the functor $\mathbb{R} \Gamma(X,-)$ to the exact sequence (5.8.4).

PRoof. Assertion (i) is a straightforward consequence of the functoriality properties of the quasi-isomorphism (5.2.1), applied to the immersion $Z \hookrightarrow X$. Assertion (ii) follows from the same functorialities, applied at the level of Čech resolutions after choosing an open covering of $Y$ by affine open subsets $U_{\alpha}$ and closed immersions $U_{\alpha} \hookrightarrow\left(\mathbb{P}_{\alpha}, F_{\alpha}\right)$, as in the proof of Theorem 5.2 (ii) given in 5.7. Finally, assertion (iii) follows from the previous one by taking global sections. 


\section{Relation with Witt vector cohomology}

We end this article with the relation between Theorem 5.2 (with its corollary 5.9) and Theorem 1.1 of [5], which identifies the slope $<1$ part of rigid cohomology with compact supports with Witt vector cohomology with compact supports.

6.1. Let $Y$ be a smooth $k$-scheme of finite type, and let $X \hookrightarrow Y$ be a closed subscheme defined by an ideal $\mathcal{I} \subset \mathcal{O}_{Y}$. Let $W_{n} \mathcal{I}:=\operatorname{Ker}\left(W_{n} \mathcal{O}_{Y} \rightarrow W_{n} \mathcal{O}_{X}\right)$, $W \mathcal{I}:=\operatorname{Ker}\left(W \mathcal{O}_{Y} \rightarrow W \mathcal{O}_{X}\right)$ be the subsheaves of Witt vectors with coefficients in $\mathcal{I}$. By construction, $\mathcal{P}_{X, Y, n}^{(0) W}$ is the divided power envelope of $W_{n} \mathcal{I}$ with compatibility with the canonical divided powers of $V W_{n-1} \mathcal{O}_{Y}$ (see (5.1.2)). Since these divided powers extend to $W_{n} \mathcal{O}_{Y} / W_{n} \mathcal{I}=W_{n} \mathcal{O}_{X}$ (as the canonical divided powers of $V W_{n-1} \mathcal{O}_{X}$ ), there are canonical ring homomorphisms

$$
\mathcal{P}_{X, Y, n}^{(0) W} \longrightarrow W_{n} \mathcal{O}_{X}, \quad \widehat{\mathcal{P}}_{X, Y}^{(0) W} \longrightarrow W \mathcal{O}_{X} .
$$

By composition with the canonical projection, we obtain a ring homomorphism

$$
\mathcal{A}_{X, Y}^{W}=\lim _{\overleftarrow{m}}\left(\widehat{\mathcal{P}}_{X, Y}^{(m) W} \otimes K\right) \rightarrow \widehat{\mathcal{P}}_{X, Y}^{(0) W} \otimes K \rightarrow W \mathcal{O}_{X, K}
$$

We can now compose with the augmentation morphism, and we obtain a morphism of complexes

$$
\mathcal{A}_{X, Y}^{W} \widehat{\otimes}_{W \mathcal{O}_{Y}} W \Omega_{Y}^{\bullet} \longrightarrow W \mathcal{O}_{X, K},
$$

where we view $W \mathcal{O}_{X, K}$ as a complex concentrated in degree 0 .

Let $Z \subset X$ be a closed subscheme defined by an ideal $\mathcal{H} \subset \mathcal{O}_{X}$, and let $U=X \backslash Z$. By functoriality, we obtain a morphism of complexes

$$
\mathcal{A}_{X \backslash Z, Y}^{\mathrm{c}, W} \widehat{\otimes}_{W \mathcal{O}_{Y}} W \Omega_{Y}^{\bullet} \longrightarrow\left(W \mathcal{O}_{X, K} \rightarrow W \mathcal{O}_{Z, K}\right)_{\mathrm{t}} .
$$

Note that the target complex is actually a resolution of $W \mathcal{H}_{K}$.

Taking the image of (6.1.2) (resp. (6.1.3)) in $D^{\mathrm{b}}(X, K)$, and composing with the isomorphism (5.2.2) (resp. (5.9.2)), we obtain canonical morphisms

$$
\begin{gathered}
\mathbb{R} \underline{\Gamma}_{\text {rig }}(X / K) \longrightarrow W \mathcal{O}_{X, K}, \\
\mathbb{R} \underline{\Gamma}_{\text {rig }, c}(X \backslash Z / K) \longrightarrow W \mathcal{H}_{K} .
\end{gathered}
$$

Assuming that $X$ is proper, and taking global sections on $X$, they define 
canonical morphisms

$$
\begin{gathered}
\mathbb{R} \Gamma_{\text {rig }}(X / K) \longrightarrow \mathbb{R} \Gamma\left(X, W \mathcal{O}_{X, K}\right), \\
\mathbb{R} \Gamma_{\text {rig, }, \mathrm{c}}(U / K) \longrightarrow \mathbb{R} \Gamma_{\mathrm{c}}\left(U, W \mathcal{O}_{U, K}\right),
\end{gathered}
$$

where the complex $\mathbb{R} \Gamma_{\mathrm{c}}\left(U, W \mathcal{O}_{U, K}\right)$ is the Witt vector cohomology with compact supports defined by [5, (2.12)].

6.2. Let $X$ be a $k$-scheme of finite type, $Z \subset X$ a closed subscheme defined by an ideal $\mathcal{H} \subset \mathcal{O}_{X}$, and $U=X \backslash Z$. In [5], morphisms

$$
\begin{gathered}
a_{X}: \mathbb{R} \underline{\Gamma}_{\text {rig }}(X / K) \longrightarrow W \mathcal{O}_{X, K}, \\
a_{X, U}: \mathbb{R} \underline{\Gamma}_{\text {rig,c }}(X \backslash Z / K) \longrightarrow W \mathcal{H}_{K}
\end{gathered}
$$

are defined in $D^{b}(X, K)$ without using the de Rham-Witt complex of a smooth ambient scheme (see [5, Prop. 4.4 and Thm. 4.5]). To compare these morphisms with (6.1.4) and (6.1.5), we now recall their construction.

Assume first that there exists a closed immersion $X \hookrightarrow \mathbb{P}$, where $\mathbb{P}$ is a smooth formal scheme endowed with a lifting $F$ of the absolute Frobenius endomorphism. Then $F$ defines a ring homomorphism [5, (4.4)]

$$
\mathcal{A}_{X, \mathrm{P}} \rightarrow W \mathcal{O}_{X, K}
$$

One can then define a morphism $\mathcal{A}_{X, \mathrm{P}} \otimes \Omega_{\mathrm{P}}^{\bullet} \rightarrow W \mathcal{O}_{X, K}$ by composition with the augmentation morphism of $\mathcal{A}_{X, \mathrm{P}} \otimes \Omega_{\mathrm{P}}^{\bullet}$ [5, (4.5)]. Using the identifications of 4.1 , its image in $D^{b}(X, K)$ does not depend on the choice of the embedding $X \hookrightarrow \mathbb{P}$, and defines a canonical morphism $a_{X}$. Using Čech resolutions as usual, the construction of $a_{X}$ can be extended to the general case where an embedding into some $(P, F)$ might not exist. Finally, the construction of $a_{X, U}$ follows using the functoriality of the construction of $a_{X}$ with respect to $Z \hookrightarrow X$.

Lemma 6.3. Let $Y$ be a smooth $k$-scheme, and let $Z \hookrightarrow X \hookrightarrow Y$ be closed subschemes. Then the morphisms (6.2.1) and (6.2.2) are respectively equal to (6.1.4) and (6.1.5).

Proof. We consider first the case where there exists a closed immersion $Y \hookrightarrow \mathbb{P}$, where $\mathbb{P}$ is a smooth formal scheme over $W$ endowed with a Frobenius lifting $F$. Then $a_{X}$ is defined as the image in $D^{\mathrm{b}}(X, K)$ of the composition

$$
a_{X, \mathrm{P}}: \mathcal{A}_{X, \mathrm{P}} \otimes_{\mathcal{O}_{\mathrm{P}}} \Omega_{\mathrm{P}}^{\bullet} \longrightarrow \mathcal{A}_{X, \mathrm{P}} \stackrel{(6.2 .3)}{\longrightarrow} W \mathcal{O}_{X, K}
$$


On the other hand, (6.1.4) is defined as the image of the composition

$$
\mathcal{A}_{X, \mathrm{P}} \otimes_{\mathcal{O}_{\mathrm{P}}} \Omega_{\mathrm{P}}^{\bullet} \underset{\mathrm{qis}}{\stackrel{(5.2 .1)}{\mathrm{q}}} \mathcal{A}_{X, Y}^{W} \widehat{\otimes}_{W \mathcal{O}_{Y}} W \Omega_{Y}^{\bullet} \longrightarrow \mathcal{A}_{X, Y}^{W} \stackrel{(6.1 .1)}{\longrightarrow} W \mathcal{O}_{X, K},
$$

where the middle arrow is the augmentation morphism. Thus it suffices to check the commutativity of the diagram of morphisms of complexes

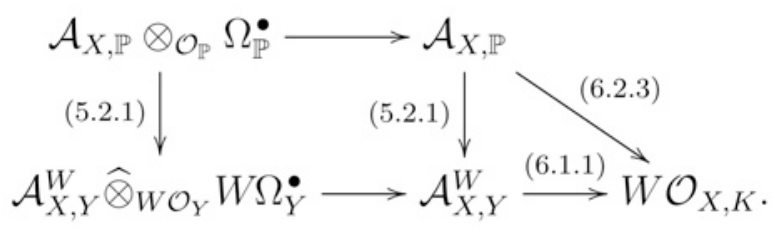

Here the middle vertical arrow is the map induced in degree 0 by (5.2.1), so the square on the left hand side obviously commutes.

To check the commutativity of the triangle, we keep the notation of 5.7. Observe first that the composition of (6.1.1) and (5.2.1) can also be written as the composition

$$
\mathcal{A}_{X, \mathrm{P}} \longrightarrow \widehat{\mathcal{P}}(\mathcal{J})_{K} \longrightarrow \widehat{\mathcal{P}}(W(\mathcal{I}))_{K} \longrightarrow W \mathcal{O}_{X, K},
$$

where the first map is given by the isomorphism (4.5.1) composed with the projection on the factor of index 0 , and the second one is induced by the morphism (3.5.3) defined by the crystal structure of $i_{\text {crys } *} \mathcal{O}_{X / W}$. On the other hand, (6.2.3) is also defined as a composition of maps starting with the homomorphism $\mathcal{A}_{X, \mathrm{P}} \rightarrow \widehat{\mathcal{P}}(\mathcal{J})_{K}$ (see [5, (4.7)]). Therefore, it suffices to check the commutativity of the triangle

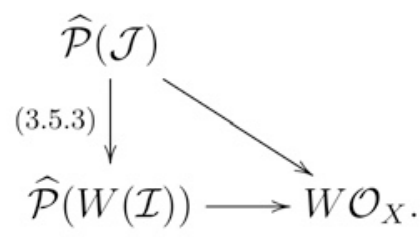

This is now a consequence of the universal property of PD-envelopes, as the oblique map is the canonical factorization of

$$
\mathcal{O}_{\mathrm{P}} \stackrel{t_{F}}{\longrightarrow} W \mathcal{O}_{P} \longrightarrow W \mathcal{O}_{X},
$$

the vertical one is the canonical factorization of

$$
\mathcal{O}_{\mathbb{P}} \stackrel{t_{F}}{\longrightarrow} W \mathcal{O}_{P} \longrightarrow W \mathcal{O}_{Y} \longrightarrow \widehat{\mathcal{P}}(W(\mathcal{I})),
$$

and the horizontal one is the canonical factorization of $W \mathcal{O}_{Y} \rightarrow W \mathcal{O}_{X}$. 
In the general case, one chooses as usual an affine open covering $\left(U_{\alpha}\right)$ of $Y$ and closed immersions $U_{\alpha} \hookrightarrow \mathbb{P}_{\alpha}$ into smooth formal schemes over $W$ endowed with Frobenius liftings $F_{\alpha}$. The previous argument can then be repeated simplicially. Taking the image in $D^{\mathrm{b}}(X, K)$ of the associated total complexes, the first assertion of the lemma follows.

The assertion relative to $X \backslash Z$ follows similarly thanks to the functoriality of diagram (6.3.1) with respect to $Z \hookrightarrow X$.

Proposition 6.4. Let $X$ be a proper $k$-scheme, $U=X \backslash Z$ an open subset of $X$, and $X \hookrightarrow Y$ a closed immersion into a smooth $k$-scheme. For each $q \geq 0$, there is a commutative diagram

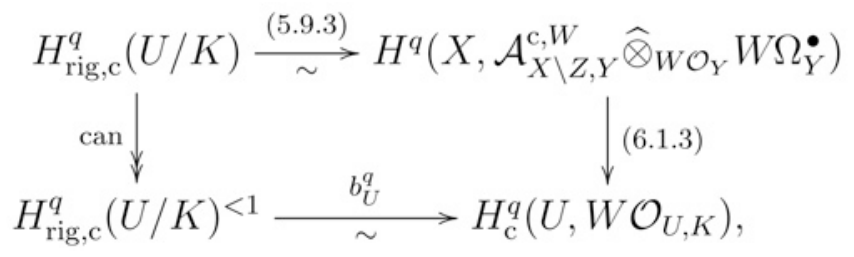

where the left vertical arrow is the projection on the slope $<1$ part, and the lower isomorphism is the canonical identification between the slope $<1$ part of rigid cohomology with compact supports and Witt vector cohomology with compact supports [5, Theorem 1.1].

Proof. Let us first recall the construction of the isomorphism $b_{U}^{q}$ (see $[5,5.2])$. Applying the functor $\mathbb{R} \Gamma(X,-)$ to the morphism $a_{U, X}$ and passing to cohomology in degree $q$, we obtain a homomorphism

$$
a_{U}^{q}: H_{\text {rig, }, \mathrm{c}}^{q}(U / K) \longrightarrow H_{\mathrm{c}}^{q}\left(U, W \mathcal{O}_{U, K}\right) .
$$

As the slopes of the Frobenius action on $H_{\mathrm{c}}^{q}\left(U, W \mathcal{O}_{U, K}\right)$ belong to [0,1[, $a_{U}^{q}$ vanishes on the subspaces of slope $\lambda$ of $H_{\text {rig,c }}^{q}(U / K)$ for any $\lambda \notin[0,1[$. Thanks to the slope decomposition of $H_{\text {rig, }, ~}^{q}(U / K)$, it follows that $a_{U}^{q}$ factorizes through $H_{\text {rig,c }}^{q}(U / K)^{<1}$, which provides the homomorphism $b_{U}^{q}$. But the previous lemma implies that $a_{U}^{q}$ is equal to the homomorphism induced on $H^{q}$ by (6.1.7). As the latter is the composition of the homomorphisms induced by (5.9.3) and (6.1.3), this proves the proposition.

Proposition 6.5. Let $Y$ be a smooth $k$-scheme of finite type, and $X \hookrightarrow Y$ a closed subscheme.

(i) The homomorphism $\mathcal{A}_{X, Y}^{W} \rightarrow W \mathcal{O}_{X, K}$ defined in (6.1.1) is an epimorphism. 
(ii) Assume that $X$ is proper over $k$, and let $H_{\text {rig }}^{q}(X / K)^{\geq 1}$ denote the component of slope $\geq 1$ of $H_{\text {rig }}^{q}(X / K)$. If we set

$$
\operatorname{Fil}^{1}\left(\mathcal{A}_{X, Y}^{W} \widehat{\otimes}_{W \mathcal{O}_{Y}} W \Omega_{Y}^{\bullet}\right):=\operatorname{Ker}\left(\mathcal{A}_{X, Y}^{W} \widehat{\otimes}_{W \mathcal{O}_{Y}} W \Omega_{Y}^{\bullet} \stackrel{(6.1 .2)}{\longrightarrow} W \mathcal{O}_{X, K}\right),
$$

the cohomology long exact sequence defined by (6.5.1) splits into short exact sequences

$$
0 \rightarrow H^{q}\left(X, \operatorname{Fil}^{1}\left(\mathcal{A}_{X, Y}^{W} \widehat{\otimes}_{W \mathcal{O}_{Y}} W \Omega_{Y}^{\bullet}\right)\right) \rightarrow H_{\text {rig }}^{q}(X / K) \rightarrow H^{q}\left(X, W \mathcal{O}_{X, K}\right) \rightarrow 0
$$

which yield isomorphisms

$$
H^{q}\left(X, \operatorname{Fil}^{1}\left(\mathcal{A}_{X, Y}^{W} \widehat{\otimes}_{W \mathcal{O}_{Y}} W \Omega_{Y}^{\bullet}\right)\right) \stackrel{\sim}{\longrightarrow} H_{\text {rig }}^{q}(X / K)^{\geq 1} .
$$

Proof. Let $\mathcal{I}$ be the ideal of $X$ in $Y$. We first fix $m \geq 0$. For each $n \geq 1$, $\mathcal{P}_{X, Y, n}^{(m) W}$ is the PD-envelope of the ideal $W_{n} \mathcal{I}^{(m)}$ with compatibility with the canonical divided powers of $V W_{n-1} \mathcal{O}_{Y}$, hence the canonical homomorphism $\mathcal{P}_{X, Y, n}^{(m) W} \rightarrow W \mathcal{O}_{X^{(m)}}$ is surjective, and its kernel is the PD-ideal $\overline{W_{n} \mathcal{I}^{(m)}}$ generated by $W_{n} \mathcal{I}^{(m)}$. It follows from $\left.[7,3.20,3)\right]$ that the homomorphisms $\overline{W_{n+1} \mathcal{I}^{(m)}} \rightarrow \overline{W_{n} \mathcal{I}^{(m)}}$ are surjective. As each $\overline{W_{n} \mathcal{I}^{(m)}}$ has vanishing cohomology over affine open subsets, the inverse system $\left(\overline{W_{n} \mathcal{I}^{(m)}}\right)_{n}$ is lim-acyclic, hence the homomorphism

$$
\widehat{\mathcal{P}}_{X, Y}^{(m) W} \longrightarrow W \mathcal{O}_{X^{(m)}}
$$

is an epimorphism, with kernel $\overline{W \mathcal{I}^{(m)}}:=\lim _{\overleftarrow{n}} \overline{W_{n} \mathcal{I}^{(m)}}$

Therefore, we obtain for variable $m$ an exact sequence of inverse systems

$$
0 \rightarrow \overline{W \mathcal{I}^{(m)}} \otimes K \rightarrow \widehat{\mathcal{P}}_{X, Y}^{(m) W} \otimes K \rightarrow W \mathcal{O}_{X^{(m)}, K} \rightarrow 0
$$

By [5, Prop. 2.1, (i)], the transition morphisms $W \mathcal{O}_{X^{(m+1)}, K} \rightarrow W \mathcal{O}_{X^{(m)}, K}$ are isomorphisms, hence assertion (i) follows if we show that the inverse system $\left(\overline{W \mathcal{I}^{(m)}} \otimes K\right)_{m}$ is lim-acyclic. The algebraic Mittag-Leffler criterion implies that the terms of this inverse system have vanishing cohomology on affine open subsets. It is then sufficient to check that, when $Y$ is affine, the system $\left.\overline{\left(W \mathcal{I}^{(m)}\right.} \otimes K\right)_{m}$ statisfies the topological Mittag-Leffler criterion. This can be done as in the proof of (5.6.2), the only difference being that, in the family of generators described in (5.6.6), we must have $\underline{k} \neq(0, \ldots, 0)$.

Using assertion (i), the definition (6.5.1) of $\mathrm{Fil}^{1}\left(\mathcal{A}_{X, Y}^{W} \widehat{\otimes}_{W \mathcal{O}_{Y}} W \Omega_{Y}^{\bullet}\right)$ provides a long exact sequence of cohomology, in which the middle terms can be identified with $H_{\text {rig }}^{q}(X / K)$ using (5.2.3). This sequence splits be- 
cause diagram (6.4.1) implies that the homomorphisms $H_{\text {rig }}^{q}(X / K) \rightarrow$ $H^{q}\left(X, W \mathcal{O}_{X, K}\right)$ are surjective for all $q$. Then the last assertion follows from [5, Th. 1.1].

REMARK 6.6. The behaviour of the long exact sequence defined by (6.5.1) is of course reminiscent of the degeneration of the slope spectral sequence for $H_{\text {rig }}^{*}(X / K) \simeq H_{\text {crys }}^{*}(X / W) \otimes K$ when $X$ is proper and smooth over $k$ [14, Th. 3.2, Cor. 3.5].

It is therefore natural (but maybe too naive) to ask the following question: assuming $X$ proper, but maybe singular, can one generalize the definition (6.5.1) so as to define a functorial filtration of $\mathcal{A}_{X, Y}^{W} \widehat{\otimes}_{W \mathcal{O}_{Y}} W \Omega_{Y}^{\bullet}$ by subcomplexes $\operatorname{Fil}^{i}\left(\mathcal{A}_{X, Y}^{W} \widehat{\otimes}_{W O_{Y}} W \Omega_{Y}^{\bullet}\right)$, depending only on $X$ in $D^{b}(X, K)$ and quasi-isomorphic to $W \Omega_{X}^{\geq i}$, when $X$ is smooth over $k$, such that their cohomology computes the slope $\geq i$ component of $H_{\text {rig }}^{q}(X / K)$ for all $q$ ?

It should be pointed out here that the work of Nakkajima [20] already provides a construction of the slope spectral sequence for $H_{\text {rig }}^{*}(X / K)$, based on the de Rham-Witt cohomology of a proper hypercovering of $X$ by smooth varieties. Nevertheless, a positive answer to the previous question would be interesting if the cohomology of the $\mathrm{Fil}^{i}$ was more accessible to computation. For example, one might hope to obtain generalizations of Katz's congruences $\bmod p^{a \kappa}$ for the number of rational points of $X$ when $X$ is a subvariety of the projective space over a finite field with $p^{a}$ elements [16]. For $\kappa=1$, these congruences are indeed a consequence of the identification of the slope $<1$ component of rigid cohomology with Witt vector cohomology [5, Cor. 1.5].

Acknowledgments. A first version of some of the results of this article was obtained while working on the joint article [5] with S. Bloch and H. Esnault. I thank them heartily for their inspiration, as well as for many stimulating discussions.

The first lecture I gave on these topics took place during a workshop on rigid cohomology and $F$-isocrystals organized jointly with $\mathrm{F}$. Baldassarri in Rennes in June 2005. It is a great pleasure for me to dedicate this article to him as an expression of my gratitude for many years of fruitful collaboration.

\section{REFERENCES}

[1] P. Berthelot, Géométrie rigide et cohomologie des variétés algébriques de caractéristique p, Journées d'Analyse p-adique (Luminy, 1982), in Introduction aux cohomologies p-adiques, Mémoires Soc. Math. France 23 (1986), pp. $7-32$. 
[2] P. Berthelot, Cohomologie rigide et cohomologie rigide à supports propres, première partie, Prépublication IRMAR 96-03, Université de Rennes (1996).

[3] P. Berthelot, D-modules arithmétiques I. Opérateurs différentiels de niveau fini, Ann. Sci. École Norm. Sup. (4) 29 (1996), pp. 185-272.

[4] P. Berthelot, Finitude et pureté cohomologique en cohomologie rigide, avec un appendice par A. J. de Jong, Invent. Math. 128 (1997), pp. 329-377.

[5] P. Berthelot - S. Bloch - H. Esnault, On Witt vector cohomology for singular varieties, Compositio Math. 143 (2007), pp. 363-392.

[6] P. Berthelot - L. Breen - W. Messing, Théorie de Dieudonné cristalline II, Lecture Notes in Mathematics 930 (1980), Springer-Verlag.

[7] P. Berthelot - A. Ogus, Notes on Crystalline Cohomology, Mathematical Notes 21 (1978), Princeton University Press.

[8] S. BLOCH, Algebraic K-theory and crystalline cohomology, Publ. Math. IHÉS 47 (1977), pp. 187-268.

[9] N. Bourbaki, Topologie générale, Chapitres 1 à 4 (1971), Springer.

[10] R. CREw, Crystalline cohomology of singular varieties, in Geometric Aspects of Dwork Theory I (2004), pp. 451-462, De Gruyter.

[11] J.-Y. Étesse, Complexe de de Rham-Witt à coefficients dans un cristal, Compositio Math. 66 (1988), pp. 57-120.

[12] A. GRoThendieck, Éléments de géométrie algébrique, Publ. Math. IHÉS 11 (1961), pp. 5-167.

[13] R. Hartshorne, On the de Rham cohomology of algebraic varieties, Publ. Math. IHÉS 45 (1975), pp. 5-99.

[14] L. Illusie, Complexe de de Rham-Witt et cohomologie cristalline, Ann. Sci. École Norm. Sup. (4) 12 (1979), pp. 501-661.

[15] N. KATZ, Nilpotent connections and the monodromy theorem: applications of a result of Turritin, Publ. Math. IHÉS 39 (1970), pp. 175-232.

[16] N. Katz, On a theorem of Ax, Amer. J. Math. 93 (1971), pp. 485-499.

[17] R. KIEHL, Theorem $A$ und Theorem $B$ in der nichtarchimedischen Funktionnentheorie, Invent. Math. 2 (1967), pp. 256-273.

[18] A. LANGer - T. Zink, De Rham-Witt cohomology for a proper and smooth morphism, J. Inst. Math. Jussieu 3 (2004), pp. 231-314.

[19] B. Le Stum, Rigid cohomology, Cambridge Tracts in Math. 172, Cambridge Univ. Press (2007).

[20] Y. NAKKAJIMA, Weight filtration and slope filtration on the rigid cohomology of a variety, to appear in Mémoires Soc. Math. France.

Manoscritto pervenuto in redazione il 22 Maggio 2012. 